OAK RIDGE

NATIONAL LABORATORY

MANAGED BY UT-BATTELLE

FOR THE DEPARTMENT OF ENERGY
Results of the Independent Radiological Verification Survey of Remediation at Building 14, Former Linde Uranium Refinery, Tonawanda, New York (LI001V)

S. P. McKenzie

M. S. Uziel

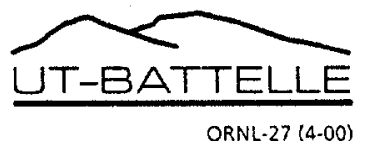


Prepared for the U.S. ARMY CORPS OF ENGINEERS, Buffalo District (Budget and Reporting Code 43WM1 1203; Agency Code DAG)

\title{
Results of the Independent Radiological Verification Survey of Remediation at Building 14, Former Linde Uranium Refinery, Tonawanda, New York (LI001V)
}

\author{
S. P. McKenzie and M. S. Uziel
}

Date Issued-July 2000

Investigation Team

R. D. Foley - Measurement Applications and Development Manager

S. P. McKenzie - Survey Team Leader

\author{
Survey Team Members \\ R. L. Coleman \\ D. E. Rice \\ R. D. Foley \\ R. C. Gosslee \\ D. A. Roberts \\ M. E. Murray \\ V.P. Patania \\ R. E. Rodriguez \\ R. R. Rodriguez \\ J. Wade \\ Work performed by the \\ MEASUREMENT APPLICATIONS AND DEVELOPMENT GROUP \\ LIFE SCIENCES DIVISION \\ Prepared by the \\ OAK RIDGE NATIONAL LABORATORY \\ Oak Ridge, Tennessee 37831-6285 \\ managed by \\ UT-BATTELLE, LLC \\ for the \\ U.S. DEPARTMENT OF ENERGY \\ under contract DE-ACO5-00OR22725
}




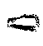




\section{CONTENTS}

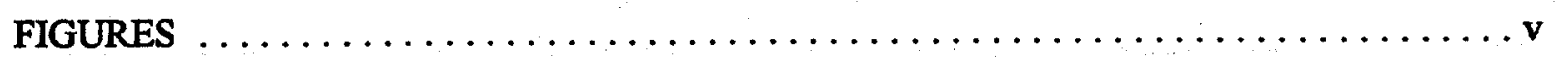

TABLES $\ldots \ldots \ldots \ldots \ldots \ldots \ldots \ldots \ldots \ldots \ldots \ldots \ldots \ldots \ldots \ldots \ldots \ldots \ldots$ vii

ACKNOWLEDGMENTS $\ldots \ldots \ldots \ldots \ldots \ldots \ldots \ldots \ldots \ldots \ldots \ldots \ldots \ldots \ldots \ldots \ldots \ldots$

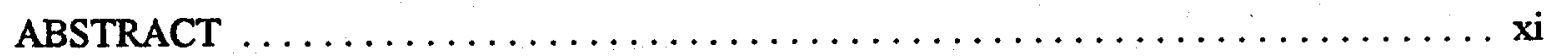

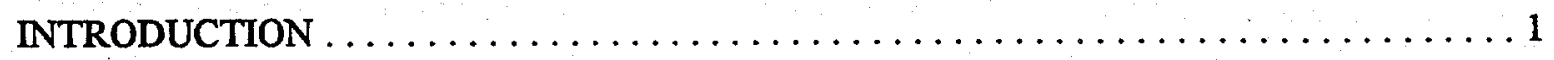

SCOPE OF THE INVESTIGATION $\ldots \ldots \ldots \ldots \ldots \ldots \ldots \ldots \ldots \ldots \ldots \ldots \ldots \ldots \ldots \ldots \ldots$

SURVEY METHODS $\ldots \ldots \ldots \ldots \ldots \ldots \ldots \ldots \ldots \ldots \ldots \ldots \ldots \ldots$

VERIFICATION SURVEY RESULTS $\ldots \ldots \ldots \ldots \ldots \ldots \ldots \ldots \ldots \ldots \ldots \ldots$

PROCESS PIPING RADIOLOGICAL INVESTIGATION $\ldots \ldots \ldots \ldots \ldots \ldots \ldots$

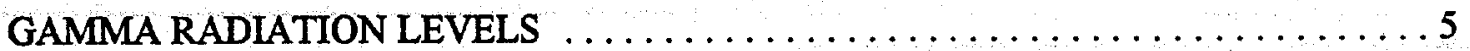

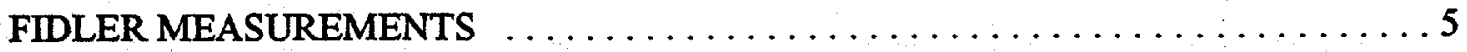

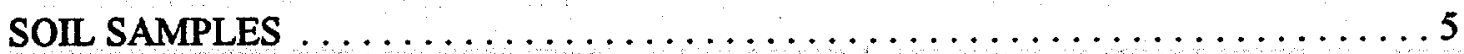

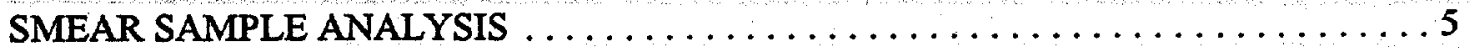

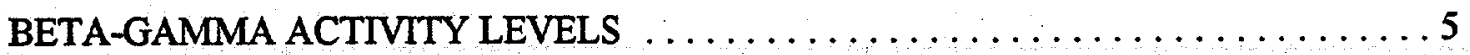

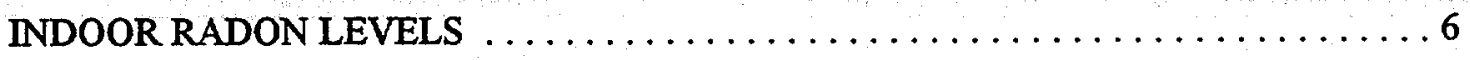

SIGNIFICANCE OF FINDINGS $\ldots \ldots \ldots \ldots \ldots \ldots \ldots \ldots \ldots \ldots \ldots \ldots \ldots$

REFERENCES $\ldots \ldots \ldots \ldots \ldots \ldots \ldots \ldots \ldots \ldots \ldots \ldots \ldots \ldots \ldots \ldots \ldots$

APPENDIX A: SUMMARY OF LOCATIONS EXCEEDING REMEDIAL

ACTION CRITERIA . . . . . . . . . . . . . . . . . . .

APPENDIX B: PROCESS PIPING RADIOLOGICAL INVESTIGATION $\ldots \ldots \ldots \ldots$ B-1

APPENDIX C: FIDLER MEASUREMENTS $\ldots \ldots \ldots \ldots \ldots \ldots \ldots \ldots \ldots \ldots \ldots \ldots \ldots$

APPENDIX D: CORRESPONDENCE $\ldots \ldots \ldots \ldots \ldots \ldots \ldots \ldots \ldots \ldots \ldots \ldots \ldots$ 


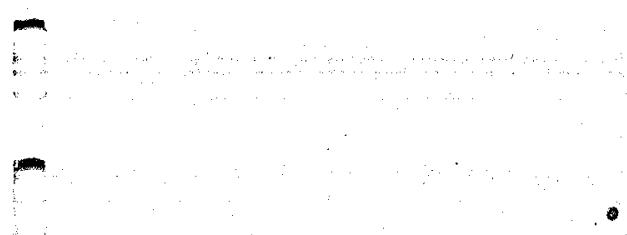

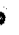

\section{FIGURES}

1 Diagram showing general location of the former Linde site in Tonawanda, New York . . . . . . . . . . . . . . . 8

2 Diagram showing general location of Building 14, at the former Linde site, Tonawanda, New York . . . . . . . . . . . . . . . 9

3 Diagram showing general layout of the first floor at Building $14 \ldots \ldots \ldots \ldots$

4 Diagram showing soil sampling locations at Building $14 \ldots \ldots \ldots \ldots \ldots \ldots \ldots$

5 Diagram showing results of radon measurements at Building $14 \ldots \ldots \ldots \ldots \ldots \ldots$ 


\section{TABLES}

1 Applicable guidelines for protection against radiation $\ldots \ldots \ldots \ldots \ldots \ldots \ldots$

2 Background radiation levels and concentrations of selected

radionuclides in soil near Tonawanda, New York $\ldots \ldots \ldots \ldots \ldots \ldots \ldots \ldots$

3 Gamma exposure rates at soil sample locations, Building 14,

former Linde Uranium Refinery, Tonawanda, New York . . . . . . . . . . 16

4 Concentrations of radionuclides in soil samples; Building 14,

former Linde Uranium Refinery, Tonawanda, New York . . . . . . . . . . . . 19

5 Transferable alpha and beta-gamma measurements at

Building 14, former Linde Uranium Refinery, Tonawanda,

New York . . . . . . . . . . . . . . . . . . . . . . . .

6 Verification survey activities summarized by area, Building 14, former Linde Uranium Refinery, Tonawanda, New York

7 Results of radon measurements in indoor air at Building 14, former Linde Uranium Refinery, Tonawanda, New York 


\section{ACKNOWLEDGMENTS}

The portion of this project completed after October 1, 1997, was sponsored by the U.S. Army Corps of Engineers, Buffalo District. Prior to that date, the project was sponsored by the U.S. Department of Energy, Office of Environmental Restoration.

The authors wish to acknowledge the contributions of R. L. Coleman, R. D. Foley, R. C. Gosslee, M. E. Murray, V. P. Patania, D. E. Rice, D. A. Roberts, R. E. Rodriguez, and J. Wade of the Measurement Applications and Development Group, Oak Ridge National Laboratory (ORNL), for sample preparation and participation in the collection, analyses, editing, and reporting of data for this survey. J. Wade of the Measurement Applications and Development Group conducted the radon sampling. R. R. Rodriguez of the ORNL Office of Radiation Protection participated in the data collection. The authors also wish to acknowledge the assistance of Mark Cafouras of Ion Technology, Inc.; Steve Nakasaki and Mike Lockler of Bechtel National, Inc.; Ron Brown and Tom Dugan of Praxair, Inc.; and Doug Davis of SEC. 


\section{ABSTRACT}

As part of the Formerly Utilized Sites Remedial Action Program, a team from Oak Ridge National Laboratory (ORNL) conducted a radiological verification survey of Building 14 at the former Linde Uranium Refinery, Tonawanda, New York. The purpose of the survey was to verify that remedial action completed by the project management contractor had reduced contamination levels to within authorized limits. Prior to remediation, fixed and removable beta-gamma emitting material was prevalent throughout Building 14 and in some of the process piping. Decontamination consisted of removal of surface contamination from floors, floor-wall interfaces, walls, wall-ceiling interfaces, and overhead areas; decontamination or removal of process piping; excavation and removal of subsurface soil; and vacuuming of dust. This independent radiological assessment was performed to verify that the remedial action had reduced contamination levels to within authorized limits.

Building 14 at the former Linde site in Tonawanda, New York, was thoroughly investigated inside for radionuclide residues. Surface residual activity levels were generally well below applicable guidelines for protection against radiation. Similarly, removable alpha and beta-gamma activity levels were below guidelines. Gamma exposure rates within the building were at typical background levels, and no elevated indoor radon concentrations were measured.

However, numerous areas exceeding U.S. Department of Energy (DOE) applicable guidelines still remain inside and underneath the building. These areas were either (1) inaccessible or (2) removal was not cost-effective or (3) removal would affect the structural integrity of the building. These above-guideline areas have been listed, described, and characterized by the remediation subcontractor (Appendix A), and dose to an exposed worker during typical exposure scenarios has been calculated. Based on the remediation subcontractor's characterization data ${ }^{1}$ and dose assessment calculations, these areas pose insignificant risk to building inhabitants under current use scenarios. However, future renovations, repairs, or demolition of the building must require prior evaluation and consideration of the areas.

Analysis of the project management contractor's post-remedial action data and results of this independent radiological verification survey by ORNL confirm that residual contamination inside the building is either below the limits prescribed by DOE applicable guidelines for protection against radiation or areas exceeding applicable guidelines have been characterized and a risk assessment completed. Building 14 can be released for unrestricted use under current use scenarios; however, arrangements must be made to inform current and future building owners of the locations of areas exceeding DOE guidelines and any associated restrictions concerning renovations, repairs, or demolition of the building.

\footnotetext{
${ }^{1}$ Radiological verification activities in these above-guideline areas were designated as outside the scope of the independent verification survey. No radiological survey activities were conducted in these areas by ORNL.
} 
-
-
-
-
-
-
- 


\section{Results of the Independent Radiological Verification Survey of the Remediation at Building 14, Former Linde Uranium Refinery, Tonawanda, New York (LI001V)'}

\section{INTRODUCTION}

From 1942 through approximately 1948, the Linde Air Products Division of Union Carbide Corporation, Tonawanda, New York, was one of many companies performing work associated with the development of nuclear energy for defense-related projects. This work was conducted under government contract to the Manhattan Engineer District (MED) and the Atomic Energy Commission (AEC). During the first 3 years, pitchblende ore from the Belgian Congo and concentrates from the Colorado Plateau ore were converted to $\mathrm{U}_{3} \mathrm{O}_{8}$. A second process yielding $\mathrm{UO}_{2}$ was conducted for about a year, and a third process, converting $\mathrm{UO}_{2}$ to green salt $\left(\mathrm{UF}_{4}\right)$, operated during World War II and the following 2 years. Linde also developed and produced barrier material for the Oak Ridge Gaseous Diffusion Plant. Other contracts have been identified, but the exact nature of the work involved is unknown (DOE 1980).

As a result of these and similar activities, equipment, buildings, and land at some of the sites became radiologically contaminated resulting in low levels of contamination on the properties. At contract termination, sites used by contractors were decontaminated in accordance with the standards and survey methods in use at that time. Since the original assessments, radiological criteria and guidelines for the release of such sites for unrestricted use have become more stringent. In some instances, records documenting decontamination efforts could not be found, and the final radiological conditions of the site could not be adequately determined. As a result, the Formerly Utilized Sites Remedial Action Program (FUSRAP) was established in 1974 to identify these formerly used sites and to reevaluate their radiological status (DOE 1980). The radiological survey detailed in this report was performed under the FUSRAP program.

The Linde site was investigated in October and November 1976 to determine the extent of on-site radiological contamination (DOE 1978). At that time, the investigation included direct measurements of alpha contamination and beta-gamma dose rates on floors, walls, ceilings, supports, and roof; collection of smear samples in the same locations to assess transferable contamination; measurement of external gamma levels; radiological analysis of exterior soil samples; and measurement of instantaneous radon concentrations. Because contamination in some areas was abovelimits set by then current federal guidelines for release of property for unrestricted use, the property was designated for remediation under FUSRAP (DOE 1978).

A remedial investigation/feasibility study-environmental impact statement process was conducted to obtain sufficient site-specific information for assessment of the nature and extent of contamination at the Tonawanda site and evaluation of remedial action alternatives (DOE 1993). This process included performing a characterization and identifying areas requiring additional investigation. Survey results at Building 14 indicated that most of the first floor contained fixed residual radioactivity

\footnotetext{
${ }^{1}$ The survey was performed by members of the Measurement Applications and Development Group of the Life Sciences Division at Oak Ridge National Laboratory.
} 
exceeding U.S. Department of Energy (DOE) guidelines ${ }^{1}$ with fixed-point beta-gamma measurements ranging from $<720$ to $280,000 \mathrm{dpm} / 100 \mathrm{~cm}^{2}$. Dust from the basement stairwell contained $590 \mathrm{pCi} / \mathrm{g}$ ${ }^{238} \mathrm{U}$. The second floor appeared to be free of contamination. Based on these results, Building 14 was scheduled for further investigation and remedial action.

In 1996, Bechtel National, Inc. (BNI), the project management contractor designated by the DOE, began remediation activities at Building 14. After significant effort by BNI, remediation activities were turned over to IDM Environmental Corporation, a turnkey remediation subcontractor under the supervision of BNI. When remediation in an area of the building was completed, an independent verification survey of the remediated area was conducted by the Measurement Applications and Development Group of ORNL. Under DOE, an independent verification contractor (IVC) was assigned to ensure the effectiveness of remedial activities performed within FUSRAP and to confirm compliance with applicable guidelines.

This report describes the independent radiological verification activities conducted intermittently by ORNL from March 1996 through January 1999 in connection with Building 14. The objectives of the verification activities were to confirm (1) that available documentation adequately and accurately described the post-remedial action status of the property that was to be verified, and (2) that remedial action reduced contamination levels to within authorized limits. Figure 1 shows the general location of the former Linde property in relation to other sites in Tonawanda. Figure 2 shows the location of Building 14 at the Linde site, and Fig. 3 show the basic floor plan of the building.

\section{SCOPE OF THE INVESTIGATION}

The radiological verification investigation included the following:

- Floor monitor ${ }^{2}$ surveys of all smooth floor areas with further characterization of any suspect contamination with hand-held beta-gamma detectors.

- Beta-gamma scans of the building interior floor areas not appropriate for the floor monitor, ${ }^{1}$ interior floor-wall interfaces, and interior walls up to $\sim 10 \mathrm{ft}$.

- Beta-gamma scans of the horizontal surfaces associated with interior overhead areas, including I-beams, cross ties, ledges, and wall-ceiling interfaces where contamination would most likely be concentrated.

- Spot checks for contamination in additions and newly remodeled areas of the building.

- Measurement of transferable alpha and beta-gamma radiation levels at selected locations in the building.

- Collection and radiological analysis of soil samples from subsurface areas exposed after removal of the concrete floor and/or excavation of contaminated soil or after drilling through the concrete slab.

- Measurement of gamma exposure rates at $1 \mathrm{~m}$ above the surface, at the surface, and at depths of 6 and $12 \mathrm{in}$. at soil sample locations.

- Systematic measurements using thin window NaI detectors (FIDLER) at 2-m intervals in Areas 8,10 , and 11 and at $1-\mathrm{m}$ intervals in Areas 20B and 20B-1.

${ }^{1} \mathrm{DOE}$ guidelines for total residual surface contamination in any one square meter for beta-gamma emitters: $5000 \mathrm{dpm} / 100 \mathrm{~cm}^{2}$ averaged over $1 \mathrm{~m}^{2}$ and $15,000 \mathrm{dpm} / 100 \mathrm{~cm}^{2}$ maximum. (More details are given in Table 1.)

${ }^{2 \star}$ "Floor monitor" described in Survey Methods section. 
- Measurement of indoor radon levels in several areas of the building using electret radon monitors.

- Examination of post-remedial action data collected by BNI and IDM Environmental Corporation and review of the post-remedial action report (BNI 1999).

A radiological survey of the building exterior and exterior surface soil and grounds in the vicinity of Building 14 was not within the scope of this investigation.

\section{SURVEY METHODS}

A comprehensive description of the survey methods and instrumentation used in this survey is given in Procedures Manual for the ORNL Radiological Survey Activities (RASA) Program, ORNL/TM-8600 (Myrick et al. 1987) and Measurement Applications and Development Group Guidelines, ORNL-6782 (ORNL 1995).

Bicron miniscaler/ratemeters with Geiger-Mueller (GM) pancake detectors were used to measure beta-gamma radiation levels. Radiation levels in counts per minute (cpm) were converted to disintegrations per minute $(\mathrm{dpm})$ per $100 \mathrm{~cm}^{2}$. Gamma radiation levels were determined using portable sodium iodide ( $\mathrm{NaI}$ ) gamma scintillation counters connected to Victoreen Model 490 Thyac III ratemeters. Because $\mathrm{NaI}$ gamma scintillators are energy dependent, measurements of gamma radiation levels in counts per minute were correlated to pressurized ionization chamber (PIC) measurements to determine gamma exposure rates in microroentgen per hour $(\mu \mathrm{R} / \mathrm{h})$ (Rodriguez et al. 1992).

Electret radon monitors manufactured by Rad Elec Inc. were used to measure radon concentrations in indoor air. The electret ion chamber contains an electrically charged Teflon ${ }^{\text {TM }}$ disk that attracts ions produced by the decay of radon and its decay products. The attracted ions cause a reduction in the electret's surface voltage. When the electret charge is measured before and after deployment, the change in total charge over the elapsed time period is proportional to the cumulative radon exposure. (Only the radon present in the room air, and not the radon progeny, can enter the electret chamber. The subsequent decay of the radon and the progeny resulting inside the chamber produces the measured ionization.)

Bicron Model GJ FIDLER detectors connected to Ludlum 2221 scaler/ratemeters were used to measure the relative gamma fluence at the surface with the purpose of detecting gamma emitting radionuclide contamination beneath poured concrete floors. The FIDLER is a NaI(TI) scintillation probe that is designed to be particularly sensitive to low-energy gamma and $x$-ray radiation. The sensitive volume is 5 in. in diameter by 0.063 in. thick and is very efficient at measuring gamma fluence rates entering perpendicular to the entrance window. The FIDLER is also sensitive to beta radiation and can be highly efficient for detecting this depending on the configuration used.

FIDLER measurements were not used for final verification purposes, but, rather, as a tool for further evaluation. FIDLER measurements were taken to assist IDM Environmental Corporation in selecting subsurface soil sampling locations. Measurements in counts per minute were taken with two different instruments. Because the results were not normalized, the observed values were compared only with other measurements taken with the same instrument.

Fifty-two systematic soil samples were collected at 49 locations after removal of the concrete slab and excavation of subsurface soil or after core drilling through concrete. Eighteen biased samples were collected at 17 locations. Systematic samples are taken from preselected or random grid locations irrespective of surface gamma exposure rates. Biased samples are collected at locations with slightly higher surface gamma exposure rates relative to surrounding areas. Concentrations of ${ }^{238} \mathrm{U},{ }^{226} \mathrm{Ra}$, and 
${ }^{232}$ Th were determined in soil samples using gamma spectrometry with high-purity germanium (HPGe) systems.

Smooth floor areas of the building were surveyed with the Ludlum Model 239-1F gas flow proportional detector system ("floor monitor"), which includes a Ludlum Model 2221 scaler/ratemeter connected to a Ludlum Model 43-37 detector probe mounted on a roll-around cart. The monitor was set in the beta mode (high voltage setting) where it is primarily used to detect beta radiation, although it is also sensitive to alpha and gamma in this mode. Anomalies detected with the floor monitor were further characterized with the GM pancake detector. Questionable spots with elevated radiation levels were sometimes analyzed on-site using a portable NaI gamma spectroscopy system. Gamma spectra were observed and compared to spectra of the radionuclides of concern.

Smear samples were obtained by wiping selected surfaces inside the building in order to assess removable alpha and beta-gamma activity levels. Samples were counted using a gross alpha smear counter and a gross beta smear counter.

A scissor lift and a man lift were used to access high overhead areas inside the building such as I-beams, cross ties, and ceiling-wall interfaces.

\section{VERIFICATION SURVEY RESULTS}

Applicable guidelines for protection against radiation are summarized in Table 1. Typical background radiation levels for the Tonawanda, New York, area are presented in Table 2. These data are provided for comparison with survey results presented in this section. Gamma radiation levels are presented in gross microroentgens per hour and FIDLER measurements in gross counts per minute. Similarly, background concentrations have not been subtracted from radionuclide concentrations in soil. Background count rates are subtracted in the conversion of alpha and beta-gamma count rates to disintegrations per minute per $100 \mathrm{~cm}^{2}\left(\mathrm{dpm} / 100 \mathrm{~cm}^{2}\right)$.

In some instances, removal of the contamination or the contaminated structure would have affected the structural integrity of the building. In others, the contamination was inaccessible or removing it was not cost-effective. Therefore, the remediation subcontractor has listed, described, and characterized these areas in a "Summary of Locations Exceeding Remedial Action Criteria" (BNI 1999), provided in Appendix A. (Figure 5-1 of BNI 1999, which is not included in this report, shows more precisely the locations of these areas.) Independent verification surveys confirmed that the areas were above applicable guidelines and that the list was complete. Characterization data collected by the remediation subcontractor and subsequent dose assessment calculations for areas exceeding remedial action criteria were reviewed by the IVC but not verified.

\section{PROCESS PIPING RADIOLOGICAL INVESTIGATION}

Process lines throughout Building 14 were evaluated and characterized for radioactive contamination by the remediation subcontractor. The methods and procedures used to conduct this evaluation and characterization were discussed with and agreed upon by the IVC. The IVC also concurred with the findings of this investigation. The first section and Attachment 1 of Summary Report for the Process Piping Radiological Investigation Praxiar Building 14 are provided in Appendix. B. [Additional attachments to this report (numbered 2-6) are not included in Appendix B.] 


\section{GAMMA RADIATION LEVELS}

Gamma exposure rates in areas where the concrete floor had been removed and the exposed soil was being sampled are shown in Table 3. Gamma exposure rates in Building 14 generally ranged from 10 to $14 \mu \mathrm{R} / \mathrm{h}$ at $1 \mathrm{~m}$ above the soil surface and from 9 to $14 \mu \mathrm{R} / \mathrm{h}$ at the surface (Table 3 ). These levels are similar to typical background levels in the Tonawanda, New York, area (Table 2). Higher surface levels up to $18 \mu \mathrm{R} / \mathrm{h}$ were measured in exposed soil with elevated ${ }^{238} \mathrm{U}$. Further excavation was conducted to remove additional soil when ${ }^{238} \mathrm{U}$ concentrations above guidelines were measured.

\section{FIDLER MEASUREMENTS}

Results of FIDLER measurements in Areas 8, 10,11, 21B, and 20B-1 are shown in Appendix C. Measurements from each detector were compared with other measurements from the same detector to locate possible subsurface contamination and potential sampling locations for IDM.

\section{SOIL SAMPLES}

Soil sample locations are shown in Fig. 4, and results of radiological analyses are listed in Table 4. Concentrations of ${ }^{238} \mathrm{U}$ in surface soil $(0-15 \mathrm{~cm})$ ranged from 0.50 to $5.5 \mathrm{pCi} / \mathrm{g}$ at 41 sample locations and from 8.1 to $670 \mathrm{pCi} / \mathrm{g}$ at 25 locations; subsurface soil $(15-30 \mathrm{~cm})$ ranged from 44 to $195 \mathrm{pCi} / \mathrm{g}$ at four sample locations. Fourteen samples were above guideline values of $30 \mathrm{pCi} / \mathrm{g}$ for ${ }^{238} \mathrm{U}$ at this site. Further excavation of soil was conducted to remove uranium-contaminated soil in Areas 12, 13,14A, and 20A after these soil samples were analyzed. Results from additional samples collected and analyzed by IDM were verified.

All accessible soil above guideline values was removed. In several areas, romoving the soil would compromise the structural integrity of the building. In these cases, the areas were described, characterized, and listed by the remediation subcontractor (BNI 1999) in the "Summary of Locations Exceeding Remedial Action Criteria" (provided in Appendix A). Included in the list are soil underneath Area 12 west, east, and south walls; soil underneath Area $14 \mathrm{~N}$ north wall and west wall; and soil underneath Area $14 \mathrm{~S}$ west wall.

Concentrations of ${ }^{226} \mathrm{Ra}$ and ${ }^{232} \mathrm{Th}$ at soil sample locations (Table 4) ranged from 0.50 to 2.0 $\mathrm{pCi} / \mathrm{g}$ and from 0.26 to $1.3 \mathrm{pCi} / \mathrm{g}$, respectively, in 70 samples from 66 locations. These levels are similar to typical background levels of ${ }^{226} \mathrm{Ra}$ and ${ }^{232} \mathrm{Th}$ found in the Tonawanda area (Table 2).

\section{SMEAR SAMPLE ANALYSIS}

Results of smear sample analysis are given in Table 5. No removable (transferable) alpha or betagamma emitting material was measured in 21 smear samples collected in 5 different areas of Building 14. All samples were less than the minimum detectable activity (MDA) of the smear counters. Removable radioactivity levels were well below applicable guidelines (Table 1).

\section{BETA-GAMMA ACTIVITY LEVELS}

Results of the surface beta-gamma scans of the floors, walls, and overhead areas on both the first and the second floor are summarized in Table 6. Detailed survey drawings are on file. Table 6 also notes other verification activities conducted in each area (e.g., collection of soil samples or smear samples, results of gamma scans, review of data collected by the remediation subcontractor, etc.). The last column of Table 6 references correspondence (included in Appendix D) releasing the area as below 
the applicable guideline limits listed in Table 1. Total residual surface contamination limits for uranium in any one square meter (Table 1) are maximum $15,000 \mathrm{dpm} / 100 \mathrm{~cm}^{2}$, average $5000 \mathrm{dpm} / 100 \mathrm{~cm}^{2}$, and removable $1000 \mathrm{dpm} / 100 \mathrm{~cm}^{2}$. Therefore, an area with scan results ranging from 3400 to $6400 \mathrm{dpm} / 100 \mathrm{~cm}^{2}$, is below guidelines if the average measurement is $\leq 5000 \mathrm{dpm} / 100 \mathrm{~cm}^{2}$ in any one square meter. Areas exceeding applicable guideline limits required further remediation.

As indicated by the survey results listed in Table 6, all areas not designated for inclusion in the "Summary of Locations Exceeding Remedial Action Criteria" (Appendix A) were below guideline limits on the date they were released by the verification contractor.

\section{INDOOR RADON LEVELS}

Twenty electret radon monitors were deployed for periods of 18 to 33 days at 17 locations between May, 28, 1998, and September 29, 1998. Sampling results are shown in Fig. 5 and Table 7. Radon concentrations in indoor air at Building 14 ranged from 0.4 to $1.6 \mathrm{pCi} / \mathrm{L}$. All measurements were well below the EPA action level of $4 \mathrm{pCi} / \mathrm{L}$.

\section{SIGNIFICANCE OF FINDINGS}

Prior to remediation, fixed and removable beta-gamma emitting material was prevalent throughout most of Building 14 and in some of the process piping. Decontamination, performed by BNI and subcontractors under the direction of BNI, consisted of removal of surface contamination from floors, floor-wall interfaces, walls, wall-ceiling interfaces, and overhead areas; decontamination or removal of process piping; excavation and removal of subsurface soil; and vacuuming of dust. This independent radiological verification survey was performed to verify that the remedial action had reduced contamination levels to within authorized limits.

Building 14 at the former Linde site in Tonawanda, New York, was thoroughly investigated inside for radionuclide residues. Surface residual activity levels were generally well below applicable guidelines for protection against radiation. Similarly, removable alpha and beta-gamma activity levels were below guidelines. Gamma exposure rates within the building were at typical background levels, and no elevated indoor radon concentrations were measured.

However, numerous areas exceeding DOE applicable guidelines still remain inside and underneath the building. These areas were either (1) inaccessible or (2) removal was not cost-effective or (3) removal would affect the structural integrity of the building. These above-guideline areas have been listed, described, and characterized by the remediation subcontractor (Appendix A), and dose to an exposed worker during typical exposure scenarios has been calculated. Based on the remediation subcontractor's characterization data ${ }^{1}$ and dose assessment calculations, these areas pose insignificant risk to building inhabitants under current use scenarios. However, future renovations, repairs, or demolition of the building must require prior evaluation and consideration of the areas.

${ }^{1}$ Radiological verification activities in these above-guideline areas (see Appendix A) were designated as outside the scope of the independent verification survey. No radiological survey activities were conducted in these areas by ORNL. 
Analysis of the project management contractor's post-remedial action data (BNI 1999) and results of this independent radiological verification survey by ORNL confirm that residual contamination inside the building is either below the limits prescribed by DOE applicable guidelines for protection against radiation or areas exceeding applicable guidelines have been characterized and a risk assessment completed. Building 14 can be released for unrestricted use under current use scenarios; however, arrangements must be made to inform current and future building owners of the locations of areas exceeding DOE guidelines and any associated restrictions concerning renovations, repairs, or demolition of the building.

\section{REFERENCES}

BNI (Bechtel National, Inc.): June 1999. Post-Remedial Action Report for Building 14 at the Linde Site, Tonowanda, New York, prepared for U.S. Army Corps of Engineers, Buffalo District, Contract No. DACW45-98-D0028.

DOE (U.S. Department of Energy). May 1978. Radiological Survey of the Former Linde Uranium Refinery, Tonawanda, New York, DOE/EV-0005/5, UC-70, U.S. Department of Energy, Division of Environmental Control Technology, Formerly Utilized MED/AEC Sites Remedial Action Program.

DOE (U.S. Department of Energy). September 1980. A Background Report for the Formerly Utilized Manhattan Engineer District/Atomic Energy Commission Sites Program, DOE/EV-0097.

DOE (U.S. Department of Energy). February 1993. Remedial Investigation Report for the Tonawanda Site, Tonawanda, New York, DOE/OR/21949-300, U.S. Department of Energy, Formerly Utilized Sites Remedial Action Program.

Myrick, T. E., et al. April 1987. Procedures Manual for the ORNL Radiological Survey Activities (RASA) Program, ORNL/TM-8600, Oak Ridge National Laboratory.

ORNL (Oak Ridge National Laboratory). January 1995. Measurement Applications and Development Group Guidelines, ORNL-6782, Oak Ridge National Laboratory.

Rodriguez, R. E., et al. October 1992. Results of the Radiological Survey at the Town of Tonawanda Landfill, Tonawanda, New York (TNY001), ORNL/RASA-92/12, Oak Ridge National Laboratory. 

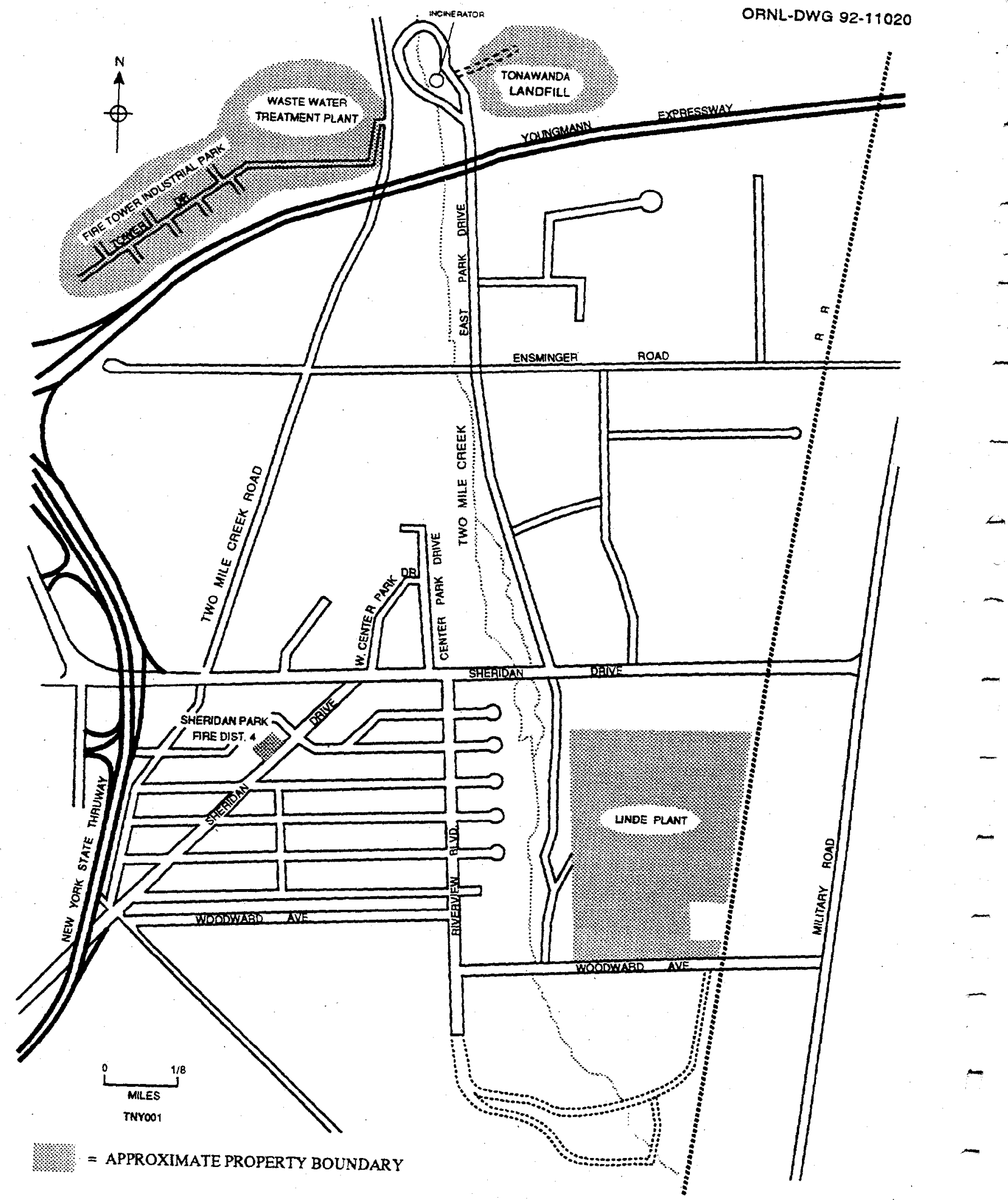

Fig. 1. Diagram showing general location of the former Linde site in Tonawanda, New York. 


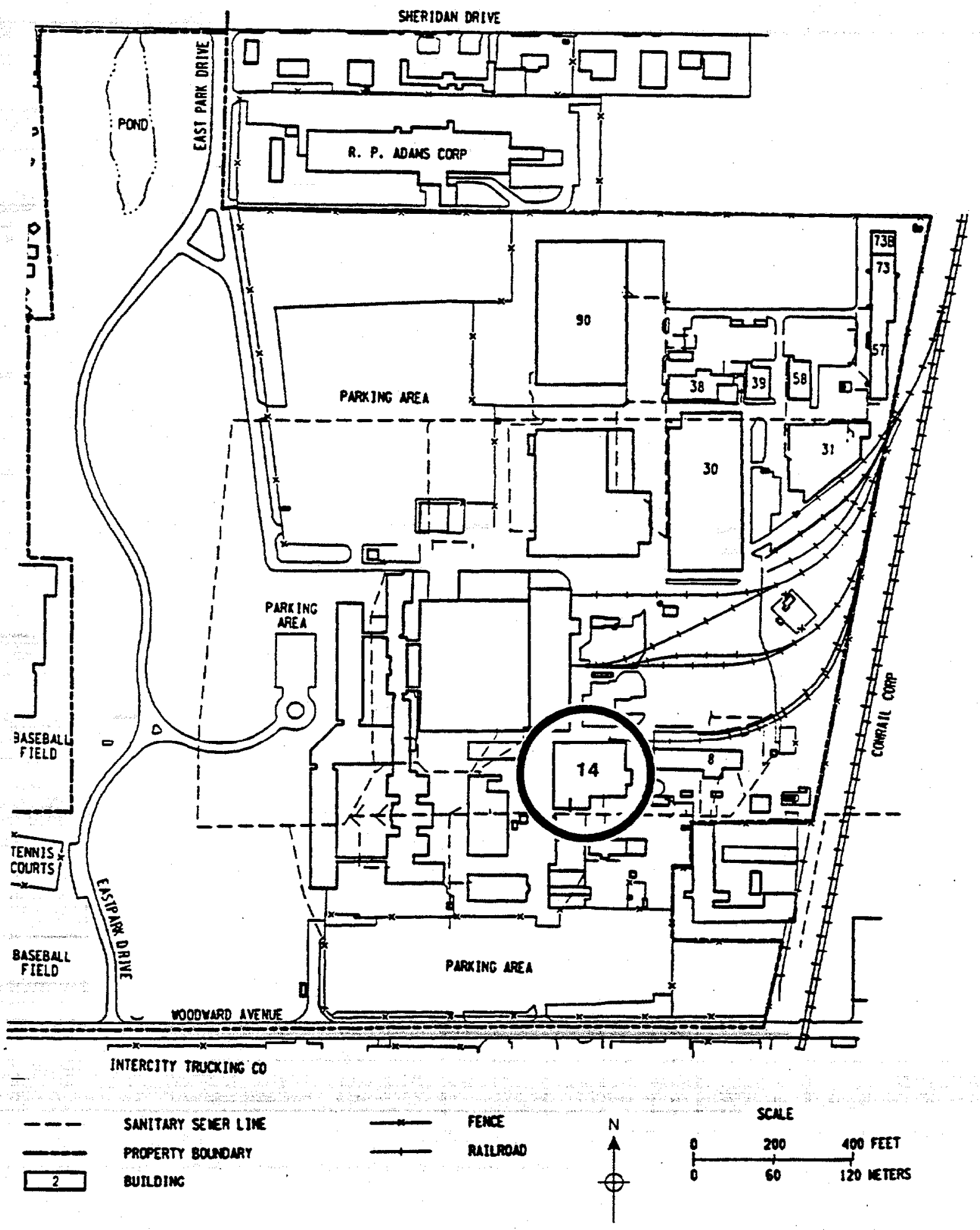

Fig. 2. Diagram showing general location of Building 14, at the former Linde site, Tonawanda, New York. 


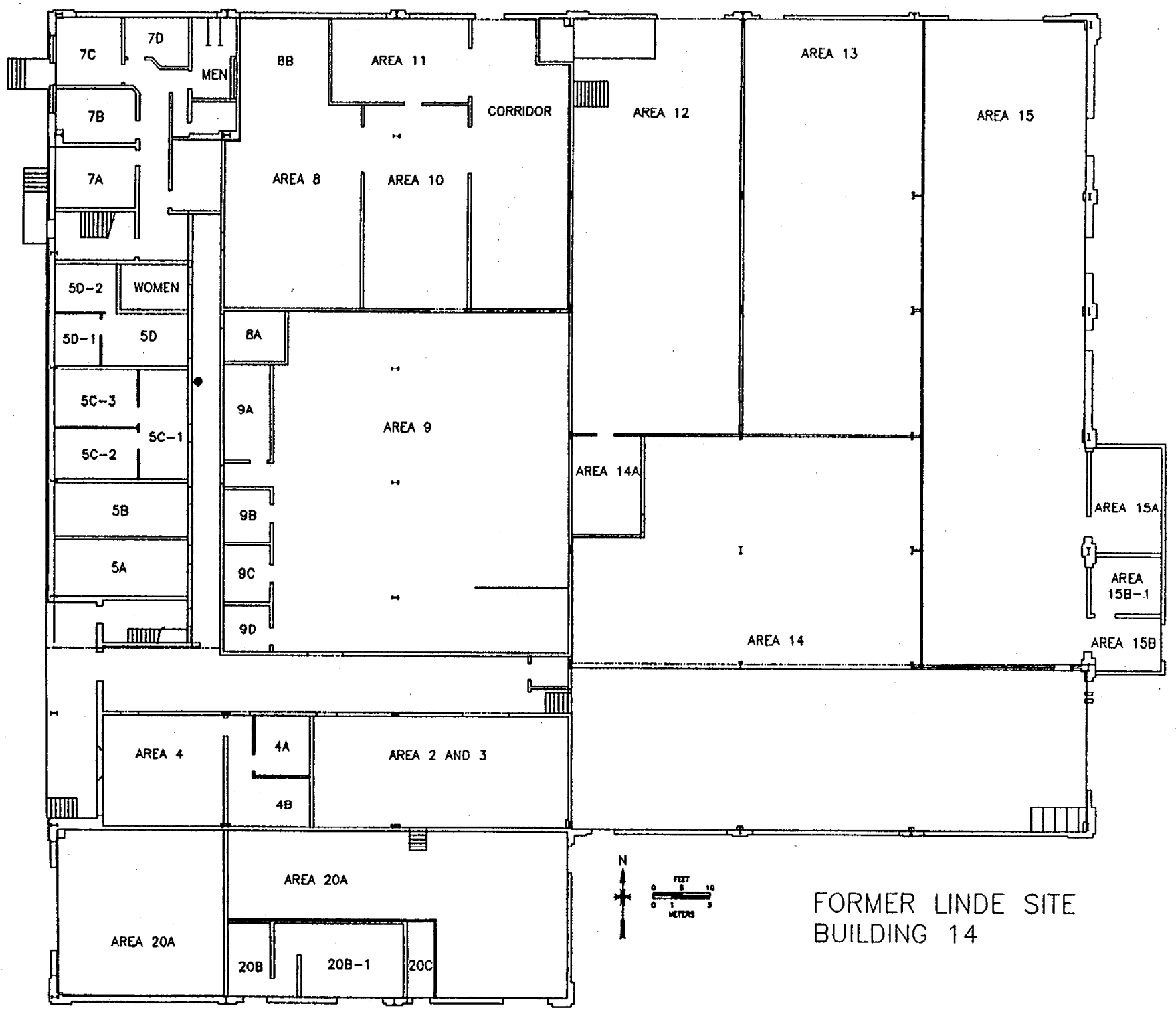

Fig. 3. Diagram showing general layout of the first floor at Building 14. 


\section{$\cdots$

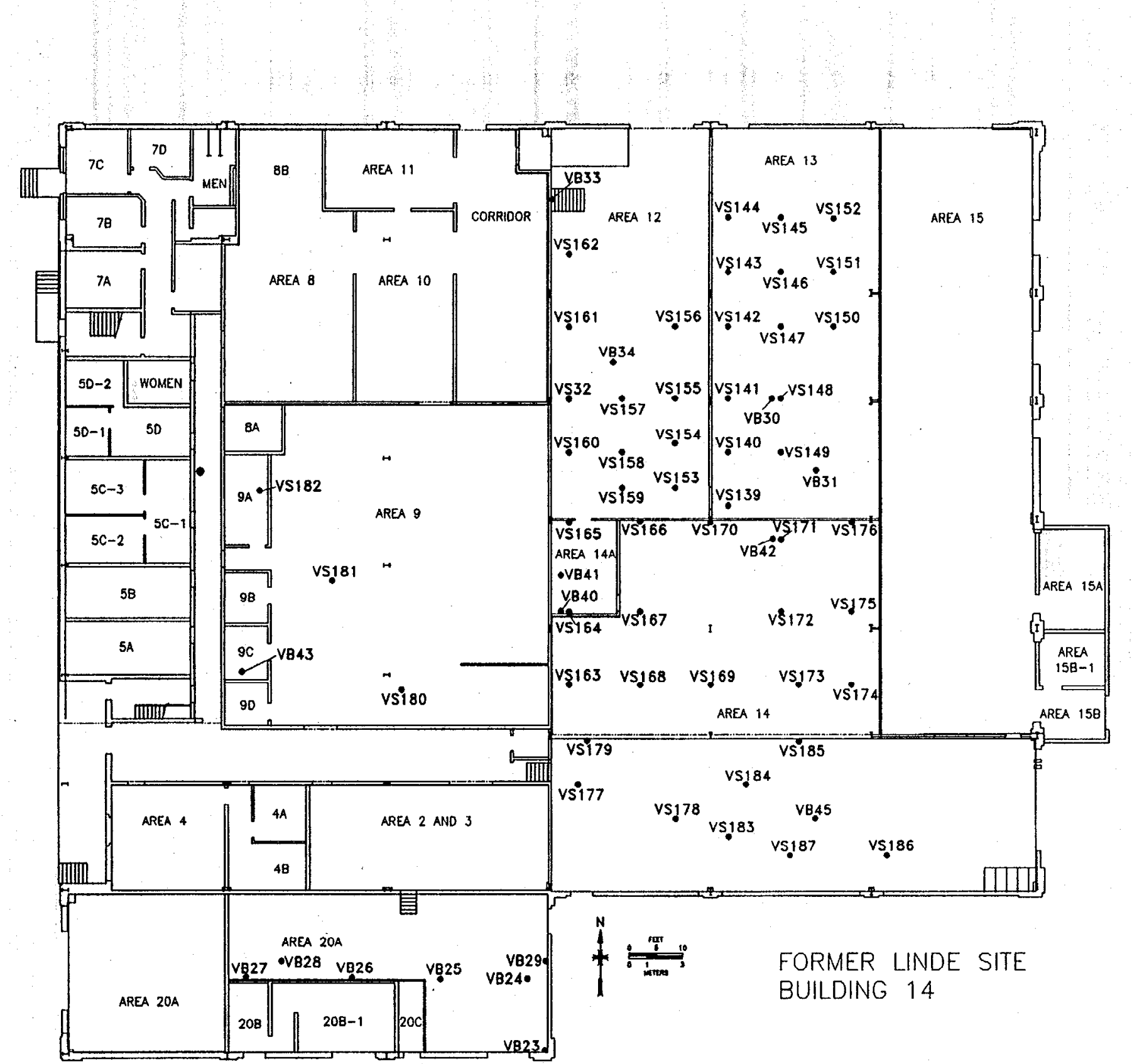

Fig. 4. Diagram showing soil sampling locations at Building 14. 


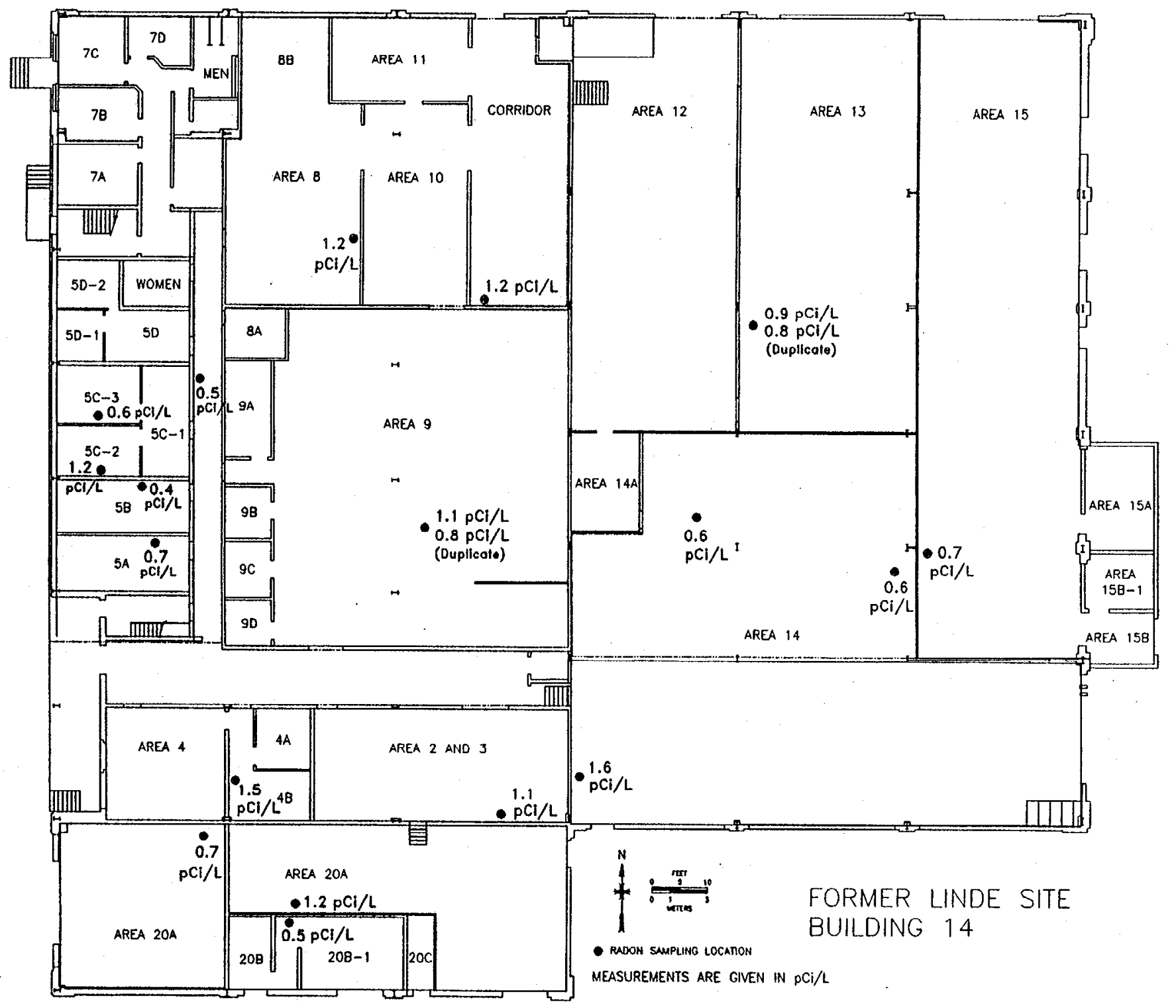

Fig. 5. Diagram showing results of radon measurements at Building 14. 
Table 1. Applicable guidelines for protection against radiation (Limits for uncontrolled areas)

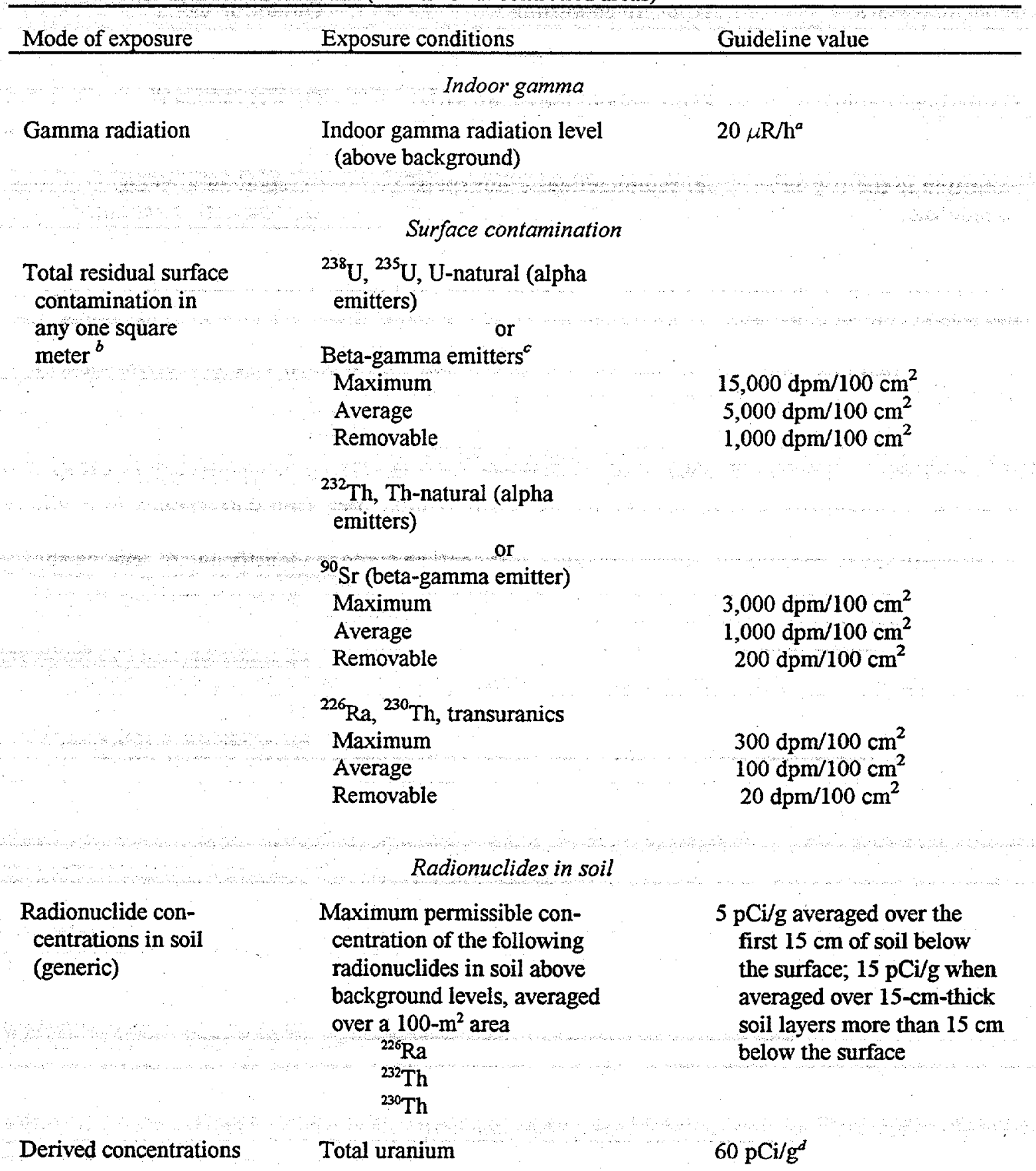


Table 1 (continued)

\begin{tabular}{|c|c|c|}
\hline exposure & xposure conditions & uidelin \\
\hline \multicolumn{3}{|c|}{ Soil hot spot criteria } \\
\hline $\begin{array}{l}\text { Guideline for non- } \\
\text { homogeneous con- } \\
\text { tamination (used in } \\
\text { addition to the } 100-\mathrm{m}^{2} \\
\text { guideline) }\end{array}$ & $\begin{array}{l}\text { Applicable to locations with } \\
\text { an area } \leq 25 \mathrm{~m}^{2} \text {, with signifi- } \\
\text { cantly elevated concentrations } \\
\text { of radionuclides ("hot spots") }\end{array}$ & $\begin{array}{l}\mathrm{G}_{A}=\mathrm{G}_{i}(100 / \mathrm{A})^{1 / 2}, \\
\text { where } \\
\mathrm{G}_{A}=\text { guideline for "hot } \\
\text { spot" of area (A) } \\
\mathrm{G}_{i}=\text { guideline averaged } \\
\text { over a } 100-\mathrm{m}^{2} \text { area }\end{array}$ \\
\hline \multicolumn{3}{|c|}{ 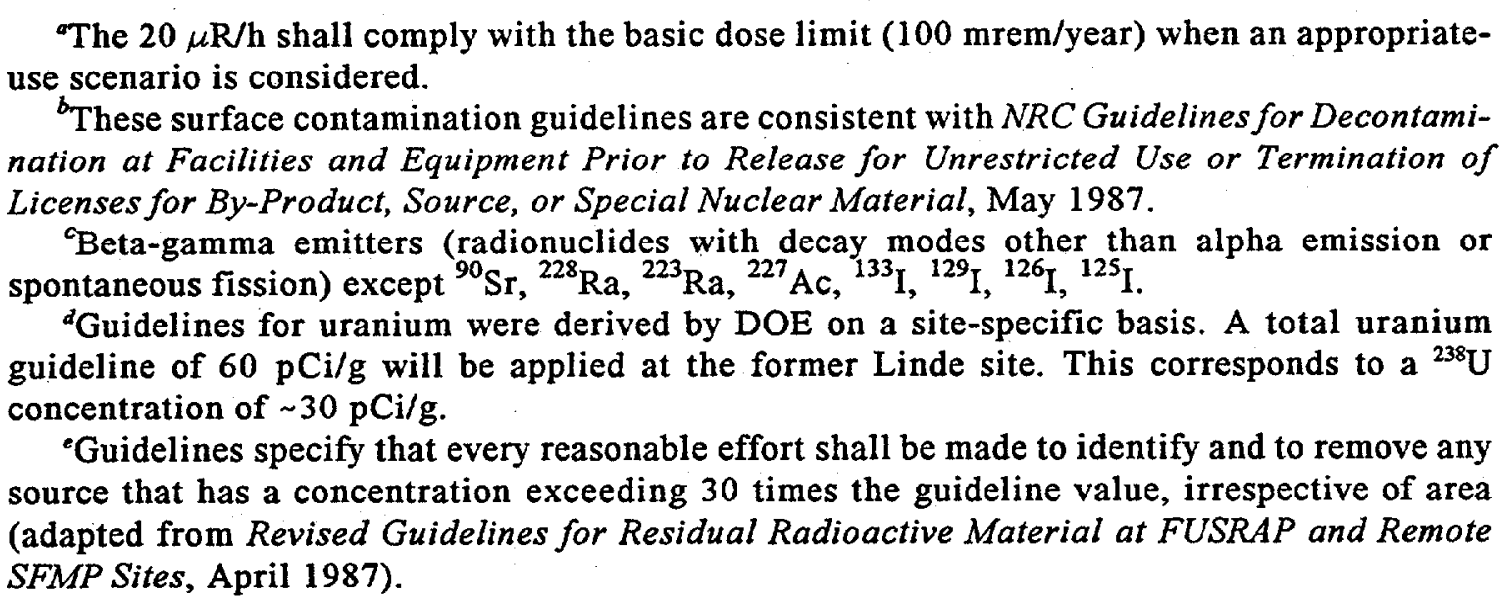 } \\
\hline $\begin{array}{l}\text { Sources: Adapted } \\
\text { partment of Energ. } \\
\text { medial Action Prog }\end{array}$ & $\begin{array}{l}\text { Department of Ene } \\
\text { nes for Residual Radi } \\
\text { Remote Surplus Faci }\end{array}$ & $\begin{array}{l}\text { 5400.5, April 1990; U.S. } \\
\text { at Formerly Utilized Sites } \\
\text { ent Program Sites, Rev. } 2 \text {, }\end{array}$ \\
\hline
\end{tabular}


Table 2. Background radiation levels and concentrations of selected radionuclides in soil near Tonawanda, New York

\begin{tabular}{|c|c|c|}
\hline \multirow{2}{*}{$\begin{array}{c}\text { Type of radiation measurement } \\
\text { or sample }\end{array}$} & \multicolumn{2}{|c|}{$\begin{array}{l}\text { Radiation level or radionuclide } \\
\text { concentration }\end{array}$} \\
\hline & Range & Average \\
\hline $\begin{array}{l}\text { Gamma exposure rate at } \\
\text { ground surface }(\mu \mathrm{R} / \mathrm{h})^{a}\end{array}$ & $8-11$ & 9 \\
\hline $\begin{array}{l}\text { Concentration of radionuclides } \\
\text { in soil }(\mathrm{pCi} / \mathrm{g})^{a} \\
{ }^{238} \mathrm{U} \\
{ }^{226} \mathrm{Ra} \\
{ }^{232} \mathrm{Th}\end{array}$ & $\begin{array}{l}0.8-1.1 \\
0.7-1.1 \\
0.5-0.9\end{array}$ & $\begin{array}{l}1.0 \\
0.9 \\
0.8\end{array}$ \\
\hline
\end{tabular}


Table 3. Gamma exposure rates at soil sample locations, Building 14, former Linde Uranium Refinery, Tonawanda, New York

\begin{tabular}{|c|c|c|c|c|c|c|}
\hline \multirow[b]{2}{*}{$\begin{array}{l}\text { Sample } \\
\mathrm{ID}^{a}\end{array}$} & \multirow[b]{2}{*}{ Area } & \multirow[b]{2}{*}{$\begin{array}{l}\text { Grid } \\
\text { Location }^{b}\end{array}$} & \multicolumn{3}{|c|}{ Gamma exposure rate $(\mu \mathrm{R} / \mathrm{h})$} & \multirow[b]{2}{*}{ Comments } \\
\hline & & & $\begin{array}{c}1 \mathrm{~m} \text { above } \\
\text { surface }\end{array}$ & Surface & $\begin{array}{c}\text { depth of } \\
6 \text { in. }\end{array}$ & \\
\hline \multicolumn{7}{|c|}{ Systematic soil samples ${ }^{c}$} \\
\hline VSI39 & 13 & $F, 10$ & $d$ & 18 & 17 & $\sim 3 \mathrm{ft}$ below grade \\
\hline VS140 & 13 & $\mathrm{I}, 10$ & 14 & 14 & 16 & $\sim 3 \mathrm{ft}$ below grade \\
\hline VS141A & 13 & $L, 10$ & 14 & 18 & 20 & $\sim 3 \mathrm{ft}$ below grade \\
\hline VS141B & 13 & $\mathrm{~L}, 10$ & 14 & $20^{e}$ & $19^{f}$ & Refusal at depth of $11 \mathrm{in}$. \\
\hline VS142A & 13 & $P, 10$ & 13 & 13 & 18 & $\sim 2 \mathrm{ft}$ below grade \\
\hline VS142B & 13 & $P, 10$ & 13 & $18^{e}$ & $18^{f}$ & Refusal at depth of $12 \mathrm{in.}$ \\
\hline VS143A & 13 & $S, 10$ & 13 & 14 & 18 & $\sim 3 \mathrm{ft}$ below grade \\
\hline VS143B & 13 & $S, 10$ & $d$ & $18^{e}$ & $18^{f}$ & \\
\hline VS144 & 13 & $V, 10$ & 13 & 13 & 13 & $\sim 3 \mathrm{ft}$ below grade \\
\hline VS145 & 13 & $V, 13$ & 13 & 13 & 14 & $\sim 2 \mathrm{ft}$ below grade \\
\hline VS146 & 13 & $S, 13$ & 13 & 13 & 14 & $\sim 2.5 \mathrm{ft}$ below grade \\
\hline VS147 & 13 & $P, 13$ & 13 & 14 & 14 & $\sim 3 \mathrm{ft}$ below grade \\
\hline VS148 & 13 & $L, 13$ & 14 & 14 & 16 & $\sim 3 \mathrm{ft}$ below grade \\
\hline VS149 & 13 & $I, 13$ & 13 & 13 & 13 & $\sim 3 \mathrm{ft}$ below grade \\
\hline VS150 & 13 & $P, 16$ & 13 & 13 & 13 & $\sim 2.5 \mathrm{ft}$ below grade \\
\hline VS151 & 13 & $S, 16$ & 13 & 13 & 13 & $\sim 2.5 \mathrm{ft}$ below grade \\
\hline VS152 & 13 & $V, 16$ & 13 & 13 & 13 & $\sim 2 \mathrm{ft}$ below grade \\
\hline VS153 & 12 & $\mathrm{G}_{0} 7$ & 15 & 15 & 14 & $\sim 3 \mathrm{ft}$ below grade \\
\hline VS154 & 12 & $\mathrm{I}+0.5,7$ & 14 & 14 & 15 & $\sim 3 \mathrm{ft}$ below grade \\
\hline VS155 & 12 & $\mathrm{~L}, 7$ & 14 & 14 & 14 & $\sim 3 \mathrm{ft}$ below grade \\
\hline VS156 & 12 & $\mathrm{P}, 7$ & 13 & 13 & 13 & $\sim 3 \mathrm{ft}$ below grade \\
\hline VS157 & 12 & $\mathrm{~L}, 4$ & 14 & 14 & 13 & $\sim 3 \mathrm{ft}$ below grade \\
\hline VS158 & 12 & 1,4 & 13 & 14 & 14 & $\sim 3 \mathrm{ft}$ below grade \\
\hline VS159 & 12 & $\mathrm{G}, 4$ & 13 & 13 & 13 & $\sim 3 \mathrm{ft}$ below grade \\
\hline VS160 & 12 & $\mathrm{I}, 1$ & 13 & 14 & 14 & $\sim 3 \mathrm{ft}$ below grade \\
\hline VS161 & 12 & $P, 1$ & 13 & 14 & 15 & $\sim 3 \mathrm{ft}$ below grade \\
\hline VS162 & 12 & $\mathrm{~T}, 1$ & 13 & 14 & 14 & $\sim 3 \mathrm{ft}$ below grade \\
\hline VS163 & $14 \mathrm{~N}$ & $\mathrm{C}, 1$ & 13 & 13 & 13 & $-4.5 \mathrm{ft}$ below grade \\
\hline VS164 & $14 \mathrm{~N}$ & $\mathrm{G}, 1$ & 13 & 13 & 14 & $\sim 4.5 \mathrm{ft}$ below grade \\
\hline
\end{tabular}


Table 3 (continued)

\begin{tabular}{|c|c|c|c|c|c|c|}
\hline \multirow{2}{*}{$\begin{array}{l}\text { Sample } \\
\mathrm{DD}^{a}\end{array}$} & \multirow[b]{2}{*}{ Area } & \multirow{2}{*}{$\begin{array}{l}\text { Grid } \\
\text { Location }^{b}\end{array}$} & \multicolumn{3}{|c|}{ Gamma exposure rate $(\mu \mathrm{R} / \mathrm{h})$} & \multirow[b]{2}{*}{ Comments } \\
\hline & & & $\begin{array}{l}1 \mathrm{~m} \text { above } \\
\text { surface }\end{array}$ & Surface & $\begin{array}{c}\text { depth of } \\
6 \mathrm{in} .\end{array}$ & \\
\hline VS165 & $14 \mathrm{~A}$ & $L, 1$ & 13 & 13 & 13 & $\sim 4 \mathrm{ft}$ below grade \\
\hline VS166 & $14 N$ & $\mathrm{~L}, 5$ & 13 & 13 & 13 & -4 t below grade \\
\hline VS167 & $14 \mathrm{~N}$ & $\mathrm{G}, 5$ & 13 & 13 & 13 & $-4 \mathrm{ft}$ below grade \\
\hline VS168 & $14 N$ & $\mathrm{C}, 5$ & 13 & 13 & 14 & $\sim 3 \mathrm{ft}$ below grade \\
\hline VS169 & $14 N$ & $\mathrm{C}, 9$ & 12 & 12 & 13 & $\sim 2 \mathrm{ft}$ below grade \\
\hline VS170 & $14 N$ & $L, 9$ & 13 & 13 & 13 & $-4 \mathrm{ft}$ below grade \\
\hline VS 172 & $14 \mathrm{~N}$ & $\mathrm{G}, 13$ & 13 & 13 & 13 & $-3 \mathrm{ft}$ below grade \\
\hline VS173 & $14 \mathrm{~N}$ & C, 14 & 11 & 13 & 13 & $-4 \mathrm{ft}$ below grade \\
\hline VS174 & $14 N$ & C, 17 & 12 & 13 & 13 & $\sim 2 \mathrm{ft}$ below grade \\
\hline VS175 & $14 \mathrm{~N}$ & $\mathrm{G}, 17$ & 13 & 13 & 13 & $\sim 3 \mathrm{ft}$ below grade \\
\hline VS176 & $14 N$ & $\mathrm{~L}, 17$ & 13 & 13 & 14 & $\sim 2 \mathrm{ft}$ below grade \\
\hline VS 177 & $14 \mathrm{~S}$ & $\mathrm{~F}, 1.5$ & 13 & 13 & 13 & $\sim 4 \mathrm{ft}$ below grade \\
\hline VS178 & $14 \mathrm{~S}$ & $D, 7$ & 12 & 12 & $g$ & $\begin{array}{l}-4 \mathrm{ft} \text { below grade, rocky, } \\
\text { water at } 6 \text { in. }\end{array}$ \\
\hline VS179 & $14 \mathrm{~S}$ & $\mathrm{I}, 2$ & 13 & 13 & 13 & $\sim 3 \mathrm{ft}$ below grade \\
\hline VS180 & 9 & $B, 10$ & 13 & 14 & 14 & $\sim 18$ in. below grade \\
\hline VS181 & 9 & $H, 6$ & 13 & 13 & 13 & $\sim 18$ in. below grade \\
\hline VS182 & $9 A$ & $M, 2$ & 13 & 13 & 10 & \\
\hline VS183 & $14 S$ & $\mathrm{C}, 10$ & 12 & 13 & 13 & $\sim 3 \mathrm{ft}$ below grade \\
\hline VS184 & $14 \mathrm{~S}$ & $\mathrm{~F}, 11$ & 12 & 12 & $d$ & $\sim 2 \mathrm{ft}$ below grade \\
\hline VS185 & $14 \mathrm{~S}$ & $\mathrm{I}, 14$ & 10 & 9 & $d$ & $\sim 1 \mathrm{ft}$ below grade \\
\hline VS186 & $14 \mathrm{~S}$ & B, 19 & 11 & 12 & 13 & $-2.5 \mathrm{ft}$ below grade \\
\hline VS187 & $14 S$ & $\mathrm{~B}, 13.5$ & Bias & soil samp & 13 & $\sim 2.5 \mathrm{ft}$ below grade \\
\hline VB23 & $20 \mathrm{~A}$ & $\begin{array}{l}\mathrm{N} \\
(-4), \mathrm{E} 18\end{array}$ & 13 & 18 & 16 & $\begin{array}{l}\text {-Sample taken below } 5 \text {-in. } \\
\text { concrete slab }\end{array}$ \\
\hline VB24 & $20 \mathrm{~A}$ & No, E17 & 12 & 13 & 13 & $\begin{array}{l}\text { Sample taken below } 12 \text {-in. } \\
\text { concrete slab }\end{array}$ \\
\hline VB25 & $20 \mathrm{~A}$ & No, E12 & 11 & 12 & 13 & $\begin{array}{l}\text { Sample taken below } 12 \text {-in. } \\
\text { concrete slab }\end{array}$ \\
\hline VB26 & $20 \mathrm{~A}$ & $\mathrm{NO}, \mathrm{E7}$ & 11 & 13 & $12^{h}$ & $\begin{array}{l}\text { Sample taken below } 12 \text {-in. } \\
\text { concrete slab }\end{array}$ \\
\hline
\end{tabular}


Table 3 (continued)

\begin{tabular}{|c|c|c|c|c|c|c|}
\hline \multirow[b]{2}{*}{$\begin{array}{l}\text { Sample } \\
\text { ID }^{a}\end{array}$} & \multirow[b]{2}{*}{ Area } & \multirow[b]{2}{*}{$\begin{array}{l}\text { Grid } \\
\text { Location }^{b}\end{array}$} & \multicolumn{3}{|c|}{ Gamma exposure rate $(\mu \mathrm{R} / \mathrm{h})$} & \multirow[b]{2}{*}{ Comments } \\
\hline & & & $\begin{array}{l}1 \mathrm{~m} \text { above } \\
\text { surface }\end{array}$ & Surface & $\begin{array}{l}\text { depth of } \\
6 \text { in. } \\
\end{array}$ & \\
\hline VB27 & $20 \mathrm{~A}$ & No, E1 & 11 & 11 & $13^{h}$ & $\begin{array}{l}\text {-Sample taken below 12-in. } \\
\text { concrete slab }\end{array}$ \\
\hline VB28 & $20 \mathrm{~A}$ & N1, E3 & $d$ & $d$ & $d$ & $\sim 2.5 \mathrm{ft}$ below grade \\
\hline VB29 & $20 \mathrm{~A}$ & N1, E18 & $d$ & $d$ & $d$ & $\sim 2 \mathrm{ft}$ below grade \\
\hline VB30 & 13 & $L, 12.5$ & 13 & 14 & 16 & $\sim 3 \mathrm{ft}$. below grade \\
\hline VB31 & 13 & $\mathrm{H}, 15$ & 13 & 14 & 16 & $\sim 3 \mathrm{ft}$ below grade \\
\hline VB32 & 12 & $\mathrm{~L}, 1$ & 14 & 18 & 18 & $\sim 3 \mathrm{ft}$ below grade \\
\hline VB33A & 12 & $\mathrm{~W}, 0$ & 13 & 14 & 18 & $-4 \mathrm{ft}$ below grade \\
\hline VB33B & 12 & $\mathrm{~W}, 0$ & 13 & $18^{e}$ & $18^{f}$ & \\
\hline VB34 & 12 & $\mathrm{~N}, 3.5$ & 13 & 14 & 14 & $\sim 3 \mathrm{ft}$ below grade \\
\hline VB40 & $14 \mathrm{~A}$ & $\mathrm{G}, 5$ & 13 & 14 & 15 & $\sim \mathrm{ft}$ below grade \\
\hline VB41 & $14 \mathrm{~A}$ & I, 5 & 13 & 14 & 14 & $-4 \mathrm{ft}$ below grade \\
\hline VB42 & $14 \mathrm{~N}$ & $\mathrm{~K}, 12.5$ & 13 & 13 & 14 & $\sim 2.5 \mathrm{ft}$ below grade \\
\hline VB43 & $9 \mathrm{C}$ & $\mathrm{C}, 1$ & 13 & 23 & 20 & \\
\hline VB45 & 14SE & $D, 15$ & $d$ & 13 & 13 & $\begin{array}{l}\sim 2 \mathrm{ft} \text { below grade; scraped } \\
\text { into wall to depth of } \sim 3 \text { in. }\end{array}$ \\
\hline
\end{tabular}

${ }^{a}$ Sample locations are shown on Fig. 4.

${ }^{b}$ Grid locations in meters measured north and east of the southwest corner of the room (NO, E0). In most rooms the number of meters north is indicated by a letter (e.g., $A=1 \mathrm{~m}, B=2 \mathrm{~m}, \ldots \mathrm{F}=6 \mathrm{~m}$, etc.) and the number of meters east by a number.

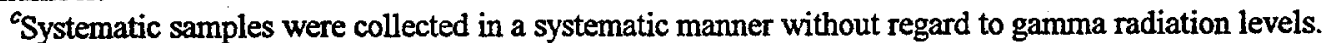

Not recorded.

Depth of 6 in.

Depth of $\sim 12$ in.

${ }^{8}$ Water at depth of 6 in.

hepth of 3 in.

'Biased samples were collected at random and at points with slightly gamma radiation levels. 
Table 4. Concentrations of radionuclides in soil samples, Building 14, former Linde Uranium Refinery, Tonawanda, New York

\begin{tabular}{|c|c|c|c|c|c|c|}
\hline \multirow{2}{*}{$\begin{array}{c}\text { Sample } \\
\mathrm{ID}^{a}\end{array}$} & \multirow{2}{*}{ Area } & \multirow{2}{*}{$\begin{array}{l}\text { Grid } \\
\text { Location }\end{array}$} & \multirow{2}{*}{$\begin{array}{l}\text { Depth } \\
(\mathrm{cm})\end{array}$} & \multicolumn{3}{|c|}{ Radionuclide concentration $(\mathrm{pCi} / \mathrm{g})^{c}$} \\
\hline & & & & ${ }^{238} \mathrm{U}$ & ${ }^{226} \mathrm{Ra}$ & ${ }^{232} \mathrm{Th}$ \\
\hline \multicolumn{7}{|c|}{ Systematic soil samples ${ }^{d}$} \\
\hline VS139 & 13 & $F, 10$ & $0-15$ & $41 \pm 2$ & $1.0 \pm 0.1$ & $0.89 \pm 0.14$ \\
\hline VS140 & 13 & 1,10 & $0-15$ & $13 \pm 1$ & $0.89 \pm 0.08$ & $0.82 \pm 0.13$ \\
\hline VS141A & 13 & $\mathrm{~L}, 10$ & $0-15$ & $53 \pm 2$ & $1.1 \pm 0.1$ & $0.99 \pm 0.13$ \\
\hline VS141B & 13 & $\mathrm{~L}, 10$ & $15-30$ & $140 \pm 10$ & $1.1 \pm 0.1$ & $1.0 \pm 0.1$ \\
\hline VS142A & 13 & $P, 10$ & $0-15$ & $20 \pm 2$ & $0.94 \pm 0.08$ & $0.95 \pm 0.13$ \\
\hline VS142B & 13 & $P, 10$ & $15-30$ & $44 \pm 2$ & $0.95 \pm 0.09$ & $0.96 \pm 0.13$ \\
\hline VSl43A & 13 & $S, 10$ & $0-15$ & $20 \pm 1$ & $0.91 \pm 0.08$ & $1.0 \pm 0.14$ \\
\hline VS143B & 13 & $\mathrm{~S}, 10$ & $15-30$ & $95 \pm 2$ & $0.94 \pm 0.09$ & $1.1 \pm 0.15$ \\
\hline VS144 & 13 & $\mathrm{~V}, 10$ & $0-15$ & $2.3 \pm 0.4$ & $0.86 \pm 0.08$ & $0.94 \pm 0.13$ \\
\hline VS145 & 13 & $\mathrm{~V}, 13$ & $0-15$ & $3.9 \pm 0.5$ & $0.94 \pm 0.08$ & $0.94 \pm 0.13$ \\
\hline VS146 & 13 & $\mathrm{~S}, 13$ & $0-15$ & $1.7 \pm 0.3$ & $0.84 \pm 0.08$ & $1.0 \pm 0.1$ \\
\hline VS147 & 13 & $P, 13$ & $0-15$ & $3.6 \pm 0.5$ & $0.92 \pm 0.08$ & $0.83 \pm 0.13$ \\
\hline VS148 & 13 & $\mathrm{~L}, 13$ & $0-15$ & $45 \pm 2$ & $0.96 \pm 0.08$ & $1.0 \pm 0.1$ \\
\hline VS149 & 13 & 1,13 & $0-15$ & $1.8 \pm 0.3$ & $0.87 \pm 0.08$ & $0.94 \pm 0.12$ \\
\hline VS150 & 13 & $P, 16$ & $0-15$ & $1.9 \pm 0.4$ & $0.73 \pm 0.07$ & $0.85 \pm 0.11$ \\
\hline VS151 & 13 & $\mathrm{~S}, 16$ & $0-15$ & $1.8 \pm 0.3$ & $0.82 \pm 0.08$ & $0.85 \pm 0.14$ \\
\hline VS152 & 13 & $V, 16$ & $0-15$ & $2.1 \pm 0.4$ & $0.99 \pm 0.08$ & $0.98 \pm 0.13$ \\
\hline VS153 & 12 & $\mathrm{G}, 7$ & $0-15$ & $8.4 \pm 0.7$ & $0.91 \neq 0.08$ & $0.96 \pm 0.13$ \\
\hline VS154 & 12 & $I+0.5,7$ & $0-15$ & $18 \pm 1$ & $0.93 \pm 0.09$ & $0.95 \pm 0.13$ \\
\hline VS155 & 12 & $\mathrm{~L}, 7$ & $0-15$ & $9.4 \pm 0.7$ & $1.1 \neq 0.1$ & $0.92 \pm 0.12$ \\
\hline VS156 & 12 & $P, 7$ & $0-15$ & $12 \pm 1$ & $0.62 \pm 0.06$ & $0.76 \pm 0.11$ \\
\hline VS157 & 12 & $\mathrm{~L}, 4$ & $0-15$ & $8.1 \pm 0.7$ & $0.91=0.08$ & $0.92 \pm 0.12$ \\
\hline VS158 & 12 & I, 4 & $0-15$ & $5.5 \pm 0.5$ & $0.93 \pm 0.08$ & $0.87 \pm 0.12$ \\
\hline VS159 & 12 & $\mathrm{G}, 4$ & $0-15$ & $4.5 \pm 0.5$ & $0.85 \pm 0.08$ & $0.85 \pm 0.11$ \\
\hline VS160 & 12 & 1,1 & $0-15$ & $12 \pm 1$ & $0.78 \pm 0.07$ & $0.88 \pm 0.12$ \\
\hline VS161 & 12 & $P, 1$ & $0-15$ & $20 \pm 1$ & $0.83 \pm 0.07$ & $0.88 \pm 0.13$ \\
\hline VS162 & 12 & $\mathrm{~T}, 1$ & $0-15$ & $12 \pm 1$ & $0.93 \pm 0.08$ & $0.85 \pm 0.12$ \\
\hline VS163 & $14 \mathrm{~N}$ & $\mathrm{C}, 1$ & $0-15$ & $1.4 \pm 0.4$ & $0.75 \pm 0.15$ & $0.58 \pm 0.08$ \\
\hline VS164 & $14 \mathrm{~N}$ & $\mathrm{G}, 1$ & $0-15$ & $12 \pm 1$ & $0.60 \pm 0.10$ & $0.60 \pm 0.08$ \\
\hline VS165 & $14 \mathrm{~A}$ & $\mathrm{~L}, 1$ & $0-15$ & $2.9 \pm 0.4$ & $0.80 \pm 0.18$ & $0.48 \pm 0.08$ \\
\hline VS166 & $14 \mathrm{~N}$ & $\mathrm{~L}, 5$ & $0-15$ & $2.0 \pm 0.4$ & $0.86 \pm 0.08$ & $0.91 \pm 0.13$ \\
\hline
\end{tabular}


Table 4 (continued)

\begin{tabular}{|c|c|c|c|c|c|c|}
\hline \multirow{2}{*}{$\begin{array}{c}\text { Sample } \\
\mathrm{DD}^{a}\end{array}$} & \multirow{2}{*}{ Area } & \multirow{2}{*}{$\begin{array}{l}\text { Grid } \\
\text { Location }^{b}\end{array}$} & \multirow{2}{*}{$\begin{array}{l}\text { Depth } \\
(\mathrm{cm})\end{array}$} & \multicolumn{3}{|c|}{ Radionuclide concentration $(\mathrm{pCi} / \mathrm{g})^{c}$} \\
\hline & & & & ${ }^{238} \mathrm{U}$ & ${ }^{226} \mathrm{Ra}$ & ${ }^{232} \mathrm{Th}$ \\
\hline VS167 & $14 \mathrm{~N}$ & G, 5 & $0-15$ & $1.8 \pm 0.4$ & $0.86 \pm 0.08$ & $0.92 \pm 0.13$ \\
\hline VS168 & $14 \mathrm{~N}$ & $\mathrm{C}, 5$ & $0-15$ & $3.3 \pm 0.8$ & $0.98 \pm 0.11$ & $1.1 \pm 0.2$ \\
\hline VS169 & $14 \mathrm{~N}$ & $\mathrm{C}, 9$ & $0-15$ & $5.5 \pm 0.8$ & $1.2 \pm 0.1$ & $1.3 \pm 0.2$ \\
\hline VS170 & $14 \mathrm{~N}$ & $L, 9$ & $0-15$ & $1.3 \pm 0.4$ & $0.89 \pm 0.09$ & $1.0 \pm 0.1$ \\
\hline VS171 & $14 \mathrm{~N}$ & $\mathrm{~K}, 13$ & $0-15$ & $2.2 \pm 0.5$ & $1.2 \pm 0.1$ & $1.1 \pm 0.1$ \\
\hline VS172 & $14 \mathrm{~N}$ & $\mathrm{G}, 13$ & $0-15$ & $3.3 \pm 0.8$ & $0.90 \pm 0.10$ & $1.0 \pm 0.2$ \\
\hline VS173 & $14 \mathrm{~N}$ & C, 14 & $0-15$ & $2.7 \pm 0.6$ & $0.60 \pm 0.10$ & $0.61 \pm 0.08$ \\
\hline VS174 & $14 \mathrm{~N}$ & $\mathrm{C}, 17$ & $0-15$ & $4.0 \pm 0.7$ & $1.4 \pm 0.1$ & $1.3 \pm 0.1$ \\
\hline VS175 & $14 \mathrm{~N}$ & $\mathrm{G}, 17$ & $0-15$ & $3.0 \pm 1.0$ & $0.70 \pm 0.15$ & $0.85 \pm 0.15$ \\
\hline VS176 & $14 \mathrm{~N}$ & $\mathrm{~L}, 17$ & $0-15$ & $1.3 \pm 0.4$ & $1.2 \pm 0.1$ & $1.2 \pm 0.1$ \\
\hline VS 177 & $14 S$ & $F, 1.5$ & $0-15$ & $1.8 \pm 0.4$ & $0.88 \pm 0.08$ & $0.92 \pm 0.12$ \\
\hline VS178 & $14 S$ & $\mathrm{D}, 7$ & $0-15$ & $4.5 \pm 1.3$ & $1.0 \pm 0.1$ & $0.96 \pm 0.13$ \\
\hline VS179 & $14 S$ & I, 2 & $0-15$ & $1.2 \pm 0.4$ & $0.81 \pm 0.08$ & $0.94 \pm 0.13$ \\
\hline VS180 & 9 & $\mathrm{~B}, 10$ & $0-15$ & $1.3 \pm 0.3$ & $1.1 \pm 0.1$ & $1.2 \pm 0.2$ \\
\hline VS181 & 9 & $\mathrm{H}, 6$ & $0-15$ & $9.5 \pm 1.0$ & $0.89 \pm 0.12$ & $1.1 \pm 0.2$ \\
\hline VS182 & $9 \mathrm{~A}$ & M, 2 & $0-15$ & $0.5 \pm 0.2$ & $0.56 \pm 0.08$ & $0.28 \pm 0.09$ \\
\hline VS183 & $14 S$ & $\mathrm{C}, 10$ & $0-15$ & $1.2 \pm 0.3$ & $0.97 \pm 0.12$ & $1.3 \pm 0.2$ \\
\hline VS184 & $14 \mathrm{~S}$ & $\mathrm{~F}, 11$ & $0-15$ & $1.9 \pm 0.7$ & $1.3 \pm 0.1$ & $1.1 \pm 0.2$ \\
\hline VS185 & $14 S$ & I, 14 & $0-15$ & $1.5 \pm 0.3$ & $0.60 \pm 0.08$ & $0.26 \pm 0.09$ \\
\hline VS186 & $14 \mathrm{~S}$ & $B, 19$ & $0-15$ & $3.8 \pm 0.5$ & $1.0 \pm 0.1$ & $1.0 \pm 0.2$ \\
\hline \multirow[t]{2}{*}{ VS187 } & $14 S$ & $\mathrm{~B}, 13.5$ & $0-15$ & $4.5 \pm 0.6$ & $1.0 \pm 0.1$ & $1.2 \pm 0.2$ \\
\hline & & & \multicolumn{2}{|c|}{ Biased soil samplese } & & \\
\hline VB23 & $20 \mathrm{~A}$ & $\begin{array}{l}N(-4), \\
\text { E18 }\end{array}$ & $0-15$ & $170 \pm 20$ & $1.6 \pm 0.1$ & $0.73 \pm 0.13$ \\
\hline VB24 & $20 \mathrm{~A}$ & No, E17 & $0-15$ & $2.3 \pm 0.4$ & $1.1 \pm 0.1$ & $0.83 \pm 0.10$ \\
\hline VB25 & $20 \mathrm{~A}$ & No, E12 & $0-15$ & $2.0 \pm 0.4$ & $0.94 \pm 0.07$ & $0.89 \pm 0.10$ \\
\hline VB26 & $20 \mathrm{~A}$ & No, E7 & $0-8$ & $9.8 \pm 1.0$ & $1.0 \pm 0.1$ & $0.37 \pm 0.07$ \\
\hline VB27 & $20 \mathrm{~A}$ & No, El & $0-8$ & $5.4 \pm 1.0$ & $1.0 \pm 0.1$ & $0.51 \pm 0.09$ \\
\hline VB28 & $20 \mathrm{~A}$ & $\mathrm{~N} 1, \mathrm{E} 3$ & $0-15$ & $0.63 \pm 0.19$ & $0.50 \pm 0.05$ & $0.28 \pm 0.06$ \\
\hline VB29 & $20 \mathrm{~A}$ & $\mathrm{~N} 1, \mathrm{E} 18$ & $0-15$ & $1.4 \pm 0.3$ & $1.6 \pm 0.1$ & $0.95 \pm 0.12$ \\
\hline VB30 & 13 & $L, 12.5$ & $0-15$ & $3.5 \pm 0.4$ & $3.0 \pm 0.1$ & $0.93 \pm 0.16$ \\
\hline
\end{tabular}


Table 4 (continued)

\begin{tabular}{|c|c|c|c|c|c|c|}
\hline \multirow{2}{*}{$\begin{array}{l}\text { Sample } \\
\mathrm{ID}^{\alpha}\end{array}$} & \multirow{2}{*}{ Area } & \multirow{2}{*}{$\begin{array}{l}\text { Grid } \\
\text { Location }^{b}\end{array}$} & \multirow{2}{*}{$\begin{array}{l}\text { Depth } \\
(\mathrm{cm})\end{array}$} & \multicolumn{3}{|c|}{ Radionuclide concentration $(\mathrm{pCi} / \mathrm{g})^{c}$} \\
\hline & & & & ${ }^{238} \mathrm{U}$ & ${ }^{226} \mathrm{Ra}$ & ${ }^{232} \mathrm{Th}$ \\
\hline VB31 & 13 & $\mathrm{H}, 15$ & $0-15$ & $30 \pm 1$ & $0.78 \pm 0.20$ & $0.39 \pm 0.11$ \\
\hline VB32 & 12 & $\mathrm{~L}, \mathrm{I}$ & $0-15$ & $33 \pm 1$ & $0.99 \pm 0.13$ & $0.91 \pm 0.18$ \\
\hline $\begin{array}{l}\text { VB33A } \\
\text { VB33B }\end{array}$ & $\begin{array}{l}12 \\
12\end{array}$ & $\begin{array}{l}\mathrm{W}, 0 \\
\mathrm{~W}, 0\end{array}$ & $\begin{array}{r}0-15 \\
15-30\end{array}$ & $\begin{array}{l}90 \pm 10 \\
55 \pm 10\end{array}$ & $\begin{array}{l}0.90 \pm 0.15 \\
0.82 \pm 0.13\end{array}$ & $\begin{array}{l}1.1 \pm 0.2 \\
1.1 \pm 0.2\end{array}$ \\
\hline VB34 & 12 & $\mathrm{~N}, 3.5$ & $0-15$ & $36 \pm 2$ & $0.96 \pm 0.14$ & $0.77 \pm 0.22$ \\
\hline $\mathrm{VB} 40^{\prime}$ & $14 \mathrm{~A}$ & $\mathrm{G}, 5$ & $0-15$ & $39 \pm 5$ & $0.85 \pm 0.10$ & $0.80 \pm 0.20$ \\
\hline VB41 & $14 \mathrm{~A}$ & 1,5 & $0-15$ & $70=15$ & $0.90=0.15$ & $0.77 \pm 0.10$ \\
\hline VB42 & $14 \mathrm{~N}$ & $\mathrm{~K}, 12.5$ & $0-15$ & $2.1 \pm 0.4$ & $0.90 \pm 0.10$ & $0.80 \pm 0.10$ \\
\hline VB43 & $9 \mathrm{C}$ & $\mathrm{C}, 1$ & $0-15$ & $670 \pm 70$ & $2.0 \pm 0.2$ & $1.3 \pm 0.3$ \\
\hline $\mathrm{VB} 45^{f}$ & $14 \mathrm{SE}$ & $D, 15$ & $0-15$ & $4.8 \pm 0.6$ & $1.0 \pm 0.1$ & $1.2 \pm 0.2$ \\
\hline
\end{tabular}

\footnotetext{
${ }^{a}$ Sample locations are shown on Fig. 4.
}

${ }^{b}$ Grid locations in meters measured north and east of the southwest corner of the room (NO, EO). In most rooms the number of meters north is indicated by a letter (e.g., $A=1 \mathrm{~m}, \mathrm{~B}=2 \mathrm{~m}, \ldots \mathrm{F}=6 \mathrm{~m}$, etc.) and the number of meters east by a number.

Indicated counting error is at the $95 \%$ confidence level $( \pm 2 \sigma)$.

${ }^{d}$ Systematic samples were collected in a systematic manner without regard to gamma radiation lcvels.

${ }^{e}$ Biased samples were collected at random and at points with slightly gamma radiation levels.

No samples numbered VB35-VB39 or VB44. 
Table 5. Transferable alpha and beta-gamma measurements at Building 14, former Linde Uranium Refinery, Tonawanda, New York

\begin{tabular}{|c|c|c|c|c|}
\hline \multirow{2}{*}{$\begin{array}{l}\text { Smear } \\
\text { sample } \\
\text { ID }\end{array}$} & \multirow{2}{*}{ Location } & \multirow{2}{*}{$\begin{array}{l}\text { Date smear } \\
\text { collected }\end{array}$} & \multicolumn{2}{|c|}{$\begin{array}{c}\text { Removable radioactivity } \\
\text { (smears) }\end{array}$} \\
\hline & & & $\begin{array}{c}\text { Alpha }{ }^{a} \\
\left(\mathrm{dpm} / 100 \mathrm{~cm}^{2}\right)\end{array}$ & $\begin{array}{l}\text { Beta-gamma } \\
\left(\mathrm{dpm} / 100 \mathrm{~cm}^{2}\right)\end{array}$ \\
\hline VT50 & Large hallway, east wall & $9-16-97$ & [0] & {$[-28]$} \\
\hline VT51 & Large hallway, west wall & $9-16-97$ & [0] & {$[-11]$} \\
\hline VT52 & $\begin{array}{l}\text { Area } 4,2 \text { m east of SW } \\
\text { corner }\end{array}$ & $9-18-97$ & [0] & {$[-39]$} \\
\hline VT53 & $\begin{array}{l}\text { Area } 4,3 \mathrm{~m} \text { north of } \mathrm{E} \\
\text { wall }\end{array}$ & $9-18-97$ & {$[0]$} & [0] \\
\hline VT54 & Large hallway; $\mathrm{NO}, \mathrm{E3}^{\mathrm{c}}$ & $9-18-97$ & {$[0]$} & {$[-6]$} \\
\hline VT55 & $\begin{array}{l}\text { Large hallway; south } \\
\text { wall; NO, E17; beam }\end{array}$ & $9-18-97$ & {$[0]$} & {$[-28]$} \\
\hline VT56 & Area $2 ; \mathrm{N} 3.5, \mathrm{E}^{\mathrm{c}}$ & 9-18-97 & {$[0]$. } & {$[-17]$} \\
\hline VT57 & Area $2 ; \mathrm{N} 3.5, \mathrm{E}^{\circ}$ & $9-18-97$ & {$[0]$} & [17] \\
\hline VT58 & Area $15,15 \mathrm{~A}$ & $11-12-97$ & {$[-7]$} & {$[-45]$} \\
\hline VT59 & Area $15,15 \mathrm{~A}$ & $11-12-97$ & {$[-7]$} & [25] \\
\hline VT60 & Area $15,15 \mathrm{~A}$ & $11-12-97$ & [0] & [0] \\
\hline VT61 & Area $15,15 \mathrm{~A}$ & $11-12-97$ & [0] & {$[-65]$} \\
\hline VT62 & Area $15,15 A$ & $11-12-97$ & [0] & {$[-90]$} \\
\hline VT63 & Area $15,15 \mathrm{~A}$ & $11-12-97$ & {$[-7]$} & {$[-40]$} \\
\hline VT64 & Area $15,15 \mathrm{~A}$ & $11-12-97$ & {$[-7]$} & {$[-10]$} \\
\hline
\end{tabular}


Table 5 (continued)

\begin{tabular}{|c|c|c|c|c|}
\hline \multirow{2}{*}{$\begin{array}{l}\text { Smear } \\
\text { sample } \\
\text { ID }\end{array}$} & \multirow{2}{*}{ Location } & \multirow{2}{*}{$\begin{array}{l}\text { Date smear } \\
\text { collected }\end{array}$} & \multicolumn{2}{|c|}{$\begin{array}{c}\text { Removable radioactivity } \\
\text { (smears) }\end{array}$} \\
\hline & & & $\begin{array}{c}\text { Alpha }^{a} \\
\left(\mathrm{dpm} / 100 \mathrm{~cm}^{2}\right)\end{array}$ & $\begin{array}{l}\text { Beta-gamma } \\
\left(\text { dpm } / 100 \mathrm{~cm}^{2}\right)\end{array}$ \\
\hline VT65 & Area $15,15 \mathrm{~A}$ & $11-12-97$ & [7] & {$[-40]$} \\
\hline VT66 & Area $15,15 \mathrm{~A}$ & $11-12-97$ & [7] & {$[-35]$} \\
\hline VT67 & Area $15,15 \mathrm{~A}$ & $11-12-97$ & {$[-7]$} & [0] \\
\hline VT70 & $\begin{array}{l}\text { Area } 14 \mathrm{~N} \text {, overhead } \\
\text { beam }\end{array}$ & $1-13-98$ & [0] & {$[-22]$} \\
\hline VT71 & Area $14 \mathrm{~N} ; \mathrm{H}, 9^{d}$ & $1-14-98$ & [0] & [28] \\
\hline VT72 & Area $14 \mathrm{~N} ; \mathrm{D}, 2^{d}$ & $1-14-98$ & {$[0]$} & {$[-39]$} \\
\hline
\end{tabular}

TMDA for alpha activity $=9 \mathrm{dpm} / 100 \mathrm{~cm}^{2}$.

MDA for beta activity $=\sim 125 \mathrm{dpm} / 100 \mathrm{~cm}^{2}$.

${ }^{\circ} \mathrm{Grid}$ locations in meters measured north and east of the southwest corner of the room (NO, E0).

${ }^{d}$ Grid location with number of meters north of southwest corner of room indicated by a letter. (e.g., $A=1 \mathrm{~m}, B=2 \mathrm{~m}, \ldots F=6 \mathrm{~m}$, etc.) and number of meters east by a number.

Note: All values represent the actual measurement less the background response of the detector used. A value in brackets [\#] indicates that the measurement was not discernable from the background response of the detector ( $95 \%$ confidence). 
Table 6. Verification survey activities summarized by area, Building 14 , former Linde Uranium Refinery, Tonawanda, New York

\begin{tabular}{|c|c|c|c|c|}
\hline Area No. ${ }^{a}$ & $\begin{array}{l}\text { Date of } \\
\text { survey }\end{array}$ & Survey results and/or comments ${ }^{b}$ & $\begin{array}{l}\text { Date released as } \\
\text { below guidelines }^{\circ}\end{array}$ & \\
\hline \multicolumn{5}{|c|}{ Second floor } \\
\hline 2nd floor & $4-14-97$ & $\begin{array}{l}\text {-Surveyed } \sim 60 \% \text { of overhead areas and } \sim 50 \% \text { of wall area. No areas elevated above guidelines. } \\
\text { - Overheads } 600-2300 \mathrm{dpm} / 100 \mathrm{~cm}^{2} \text {. } \\
\text { - Walls } 600-1500 \mathrm{dpm} / 100 \mathrm{~cm}^{2} \text {. } \\
\text {-2nd floor added after Manhattan Engineering District activities. No contamination suspected on floors } \\
\text { and internal walls that have not been surveyed. } \\
\text {-2nd floor complcted }\end{array}$ & & \\
\hline 2nd floor & $4-15-97$ & $\begin{array}{l}\text { •Random FIDLER measurements. Nothing above typical background. Completes 2nd floor. } \\
\text { First floor }\end{array}$ & 2nd floor 5-5-97 & \\
\hline $\begin{array}{l}2,3,4, \& \\
\text { large } \\
\text { hallway }\end{array}$ & $\begin{array}{l}9-15-97 \\
9-16-97\end{array}$ & -Walls surveyed. No elevated areas. & & N \\
\hline 2 & $\begin{array}{l}9-15-97 \\
9-16-97\end{array}$ & $\begin{array}{l}\cdot \text { Overheads } 300-1800 \mathrm{dpm} / 100 \mathrm{~cm}^{2} \\
\cdot \text { Walls } 1200-2100 \mathrm{dpm} / 100 \mathrm{~cm}^{2} \\
\text {-Floor monitor. No anomalies. } \\
\text {-Beta-gamma pancake survey } 600-1800 \mathrm{dpm} / 100 \mathrm{~cm}^{2} \\
\text {-Smears VT56, VT57. }\end{array}$ & Area 2 10-27-97 & \\
\hline 3 & 9-15-97 & $\begin{array}{l}\text {-Walls } 750-1800 \mathrm{dpm} / 100 \mathrm{~cm}^{2} \text {. } \\
\text {-No anomalies. }\end{array}$ & & \\
\hline 3 & $9-18-97$ & $\begin{array}{l}\text {-Floors, } 600-1500 \mathrm{dpm} / 100 \mathrm{~cm}^{2} \text {; corners and edging } 600-1800 \mathrm{dpm} / 100 \mathrm{~cm}^{2} \text {. } \\
\text { Overheads } 600-1200 \mathrm{dpm} / 100 \mathrm{~cm}^{2} \text {. } \\
\text {-No anomalies. }\end{array}$ & Area 3 10-27-97 & \\
\hline 4 & $\begin{array}{l}9-15-97 \\
9-16-97\end{array}$ & $\begin{array}{l}\cdot \text { Overheads, } 900-2100 \mathrm{dpm} / 100 \mathrm{~cm}^{2} \\
\cdot \text { Walls, } 750-1800 \mathrm{dpm} / 100 \mathrm{~cm}^{2} \\
\text {-Floors, } 600-1800 \mathrm{dpm} / 100 \mathrm{~cm}^{2} \text {; smears VT52 and VT53. } \\
\text { 'No anomalies. }\end{array}$ & Area 4 10-27-97 & \\
\hline
\end{tabular}




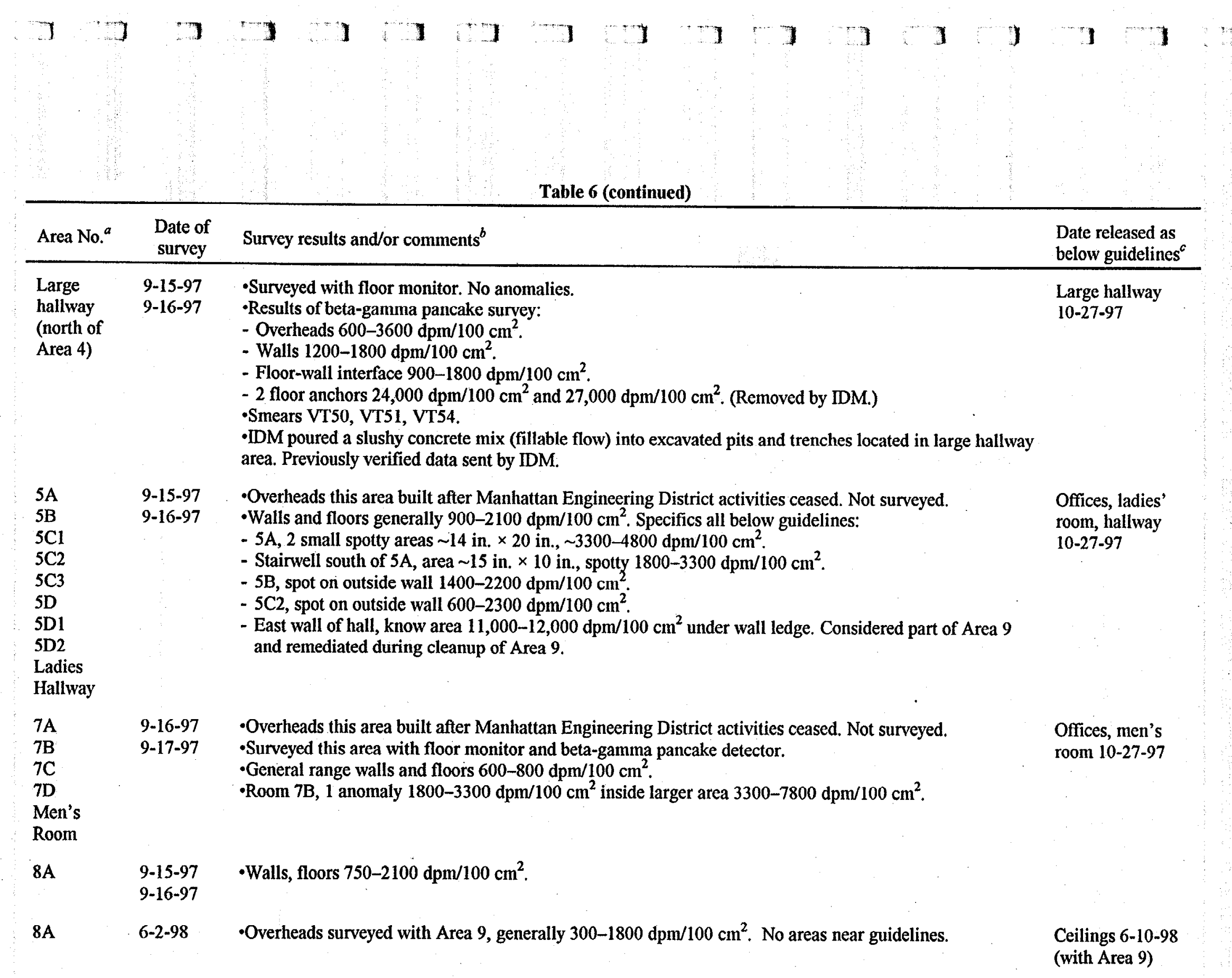


Table 6 (continued)

\begin{tabular}{|c|c|c|c|}
\hline Area No. ${ }^{a}$ & $\begin{array}{l}\text { Date of } \\
\text { survey }\end{array}$ & Survey results and/or comments ${ }^{b}$ & $\begin{array}{l}\text { Date released as } \\
\text { below guidelines }\end{array}$ \\
\hline $8 \mathrm{~A}$ & $7-16-98$ & -Floor and 3 walls below guidelines, $300-1200 \mathrm{dpm} / 100 \mathrm{~cm}^{2}$. & $\begin{array}{l}\text { Other areas } \\
9-16-98 \\
\text { (with Area 9) }\end{array}$ \\
\hline 8 & 4-14-97 & -Completed accessible overhead area $(\sim 30 \%$ area blocked $) ; 600-1800 \mathrm{dpm} / 100 \mathrm{~cm}^{2}$. & \\
\hline 8 & $4-15-97$ & $\begin{array}{l}\text {-Blocked area cleared. Completed survey. } \\
\text { - Floors } 600-2700 \mathrm{dpm} / 100 \mathrm{~cm}^{2} \text {. } \\
\text { - Overheads } 600-1800 \mathrm{dpm} / 100 \mathrm{~cm}^{2} \text { with one spot } 11,000 \mathrm{dpm} / 100 \mathrm{~cm}^{2} \text {. This is a small spot that meets } \\
\text { guidelines. }\end{array}$ & $\begin{array}{l}\text { Above-ground } \\
\text { surfaces 5-5-97; } \\
\text { Subsurface } \\
10-27-97\end{array}$ \\
\hline $8,10,11$ & $4-15-97$ & -FIDLER measurements on 2-m grid. Results provided 2 sampling locations for IDM & $\begin{array}{l}\text { Above-ground } \\
\text { surfaces 5-5-97; } \\
\text { Subsurface } \\
\text { 10-27-97; } \\
\text { Remaining areas } \\
7-7-98\end{array}$ \\
\hline $9 \mathrm{Lab}$ & $6-2-98$ & $\begin{array}{l}\text { Overheads. } \\
\text { - Scaffolding erected with walkboards. } \\
\text { - } 40 \% \text { of horizontal surfaces surveyed concentrating on I-beams and other structures most likely in place } \\
\text { during Manhattan Engineering District activities. } \\
\text { - Much of } 9 \text { Lab overhead inaccessible due to ventilation system. } \\
\text { - Generally } 300-1800 \mathrm{dpm} / 100 \mathrm{~cm}^{2} \text {. Few spots } 4800-6300 \mathrm{dpm} / 100 \mathrm{~cm}^{2} \text {. No areas near guidelines. }\end{array}$ & $\begin{array}{l}\text { Ceilings and } \\
\text { overheads } \\
6-10-98\end{array}$ \\
\hline
\end{tabular}


Table 6 (continued)

\begin{tabular}{|c|c|c|c|}
\hline Area No. ${ }^{a}$ & $\begin{array}{l}\text { Date of } \\
\text { survey }\end{array}$ & Survey results and/or comments ${ }^{b}$ & $\begin{array}{l}\text { Date released as } \\
\text { below guidelines }\end{array}$ \\
\hline 9 & $7-9-98$ & $\begin{array}{l}\text {-Surveyed floor and subsurfaces, } 13-16 \mu \mathrm{R} / \mathrm{h} \text {. Below guidelines with the following exceptions. } \\
\text { - Floor underneath two large hoods on east side of room will remain as contaminated surfaces. } \\
\text { - Room } 9 \mathrm{~B}, \sim 3-\mathrm{m}^{2} \text { area on } \mathrm{N} \text { wall up to } 17,000 \mathrm{dpm} / 100 \mathrm{~cm}^{2} \text {; typically } 9000-11,000 \text { or } \\
12,000 \mathrm{dpm} / 100 \mathrm{~cm}^{2} \text {. (Wall removed by } \mathrm{IDM} \text {.) } \\
\text { - Room } 9 \mathrm{D}, \text { end of one pipe }=71,000 \mathrm{dpm} / 100 \mathrm{~cm}^{2} \text {. (Not cost-effective to remove. }{ }^{d} \text { ) } \\
\text { - Area on south wall at location } \mathrm{A}, 10 \text { ranged up to } 32,000 \mathrm{dpm} / 100 \mathrm{~cm}^{2} \text { (generally } \\
11,000-17,000 \mathrm{dpm} / 100 \mathrm{~cm}^{2} \text {. (Wall removed by IDM.) } \\
\text { - } 4 \text { lead anchors up to } 53,000 \mathrm{dpm} / 100 \mathrm{~cm}^{2} ; \text { lots of non-contaminated lcad anchors. (Removed by IDM.) } \\
\text { - Column at location } \mathrm{D}, 9 \mathrm{contaminated} \mathrm{around} \mathrm{base} 17,000-44,000 \mathrm{dpm} / 100 \mathrm{~cm}^{2} \text {. (Removed by IDM.) } \\
\text { - Hottest spot in trench }=23 \mu \mathrm{R} / \mathrm{h}, 130,000 \mathrm{dpm} / 100 \mathrm{~cm}^{2} \text {. (Removed by IDM.) } \\
\text { - Biased soil sample VB43 containing } 670 \mathrm{pCi} / \mathrm{g}^{238} \mathrm{U} \text { collected at this location (C, 1). (Area excavated } \\
\text { further by IDM.) } \\
\text { - Systematic soil samples VS180-VS182 collected in Area } 9 \text {; VS181 contained } 9.5 \mathrm{pCi} / \mathrm{g}{ }^{238} \mathrm{U} \text {; others } \\
\text { similar to typical background. }\end{array}$ & \\
\hline 9 & $7-16-98$ & $\begin{array}{l}\text {-After additional excavation, IDM supplied soil sample data to ORNL for verification. } \\
\text {-Inside walls with associated contamination had been removed by IDM. }\end{array}$ & $\begin{array}{l}\text { Exposed soil, } \\
\text { concrete floor, } \\
\text { walls 9-16-98; } \\
\text { Soil samples } \\
10-23-98\end{array}$ \\
\hline 10 & 4-14-97 & $\begin{array}{l}\text {-Surveyed } \sim 50 \% \text { of overheads, } \sim 70 \% \text { of floors, and } 1 \mathrm{~m} \text { up on wall. } \\
\text { - Floors } 600-3600 \mathrm{dpm} / 100 \mathrm{~cm}^{2} . \\
\text { - Overheads } 600-1800 \mathrm{dpm} / 100 \mathrm{~cm}^{2} \text {. } \\
\text { - Walls, } 2 \text { spots with elevated measurements within guidelines. No action needed. } \\
\text { (1) East wall upper horizontal surface, } 1 \mathrm{~m} \times 30 \mathrm{~cm}=5000 \mathrm{dpm} / 100 \mathrm{~cm} 2 \\
\text { (2) South wall upper horizontal surface } 1 \mathrm{~cm} \times 75 \mathrm{~cm}=5000-7500 \mathrm{dpm} / 100 \mathrm{~cm}^{2} \text {. }\end{array}$ & $\begin{array}{l}\text { Above-ground } \\
\text { surfaces } 5-5-97 \\
\text { Subsurface } \\
10-27-97\end{array}$ \\
\hline 11 & 4-14-97 & $\begin{array}{l}\text {-Surveyed } \sim 50 \% \text { of overheads, } \sim 70 \% \text { of floors, and } 1 \mathrm{~m} \text { up on wall. } \\
\text { - Floors } 600-2700 \mathrm{dpm} / 100 \mathrm{~cm}^{2} \\
\text { - Overheads } 600-1800 \mathrm{dpm} / 100 \mathrm{~cm}^{2}\end{array}$ & \\
\hline
\end{tabular}
6-98

Above-ground

Floors 600-3600 dpm/100 $\mathrm{cm}^{2}$.

.

(1) East wall upper horizontal surface, $1 \mathrm{~m} \times 30 \mathrm{~cm}=5000 \mathrm{dpm} / 100 \mathrm{~cm}^{2}$.

Subsurface

Surveyed $\sim 50 \%$ of overheads, $\sim 70 \%$ of floors, and $1 \mathrm{~m}$ up on wall.

(1) $600-2700 \mathrm{dpm} / 100 \mathrm{~cm}^{2}$ 
Table 6 (continued)

\begin{tabular}{|c|c|c|c|}
\hline Area No. ${ }^{a}$ & $\begin{array}{l}\text { Date of } \\
\text { survey }\end{array}$ & Survey results and/or comments ${ }^{b}$ & $\begin{array}{l}\text { Date released as } \\
\text { below guidelines }\end{array}$ \\
\hline 11 & 4-15-97 & -Surveyed $30 \%$ walls. No anomalies & $\begin{array}{l}\text { Above-ground } \\
\text { surfaces 5-5-97; } \\
\text { Subsurface } \\
10-27-97\end{array}$ \\
\hline Corridor & $8-28-97$ & $\begin{array}{l}\text {-Floor (300-1500 with exception of a few elevated areas that will be remediated) below guidelines. } \\
\text {-Walls } 1800-2700 \text { due to high background from brick. } \\
\cdot \text { Overheads } 0-900 \mathrm{dpm} / 100 \mathrm{~cm}^{2} \text { (only looked at certain areas). }\end{array}$ & $\begin{array}{l}10-27-97 \\
7-7-98\end{array}$ \\
\hline $\begin{array}{l}\text { Stairwell } \\
\text { leading to } \\
\text { utility } \\
\text { tunnel in } \\
\text { Area } 12\end{array}$ & $5-4-98$ & $\begin{array}{l}\cdot 0-1200 \mathrm{dpm} / 100 \mathrm{~cm}^{2} . \\
\cdot \text { Overhead electrical conduits elevated on top of the steam line. IDM will remediate. }\end{array}$ & $\begin{array}{l}5-20-98 \\
9-21-98\end{array}$ \\
\hline $\begin{array}{l}\text { Stairwell } \\
\text { leading to } \\
\text { utility } \\
\text { tunnel }\end{array}$ & & -Walls, stairs, floor, ceiling, and electrical conduits. & $10-23-98$ \\
\hline $\begin{array}{l}\text { Pipes in } \\
\text { utility } \\
\text { tunnel near } \\
\text { Area } 12\end{array}$ & $5-6-98$ & $\begin{array}{l}\text {-IDM to conduct more decon work on pipes in this area. After decon, IDM supplied data to ORNL for } \\
\text { verification. }\end{array}$ & $9-21-98$ \\
\hline $\begin{array}{l}\text { Pipe in } \\
\text { Area } 12 \\
\text { sump } \\
\text { (tunnel } \\
\text { access) }\end{array}$ & $7-16-98$ & $\cdot$ Re-surveyed after decon, $300-1800 \mathrm{dpm} / 100 \mathrm{~cm}^{2}$; highest spot $3300 \mathrm{dpm} / 100 \mathrm{~cm}^{2}$. & $9-21-98$ \\
\hline $\begin{array}{l}\text { Sump in } \\
\text { utility } \\
\text { tunnel }\end{array}$ & & $\begin{array}{l}\text {-Reviewed IDM data. } \\
\text { - Released surface of sump and east and west drain lines. } \\
\text { - North drain line exceeds DOE criteria. }{ }^{d}\end{array}$ & $11-10-98$ \\
\hline
\end{tabular}

- Released surface of sump and east and west drain lines.

-North drain line exceeds DOE criteria. ${ }^{d}$ 


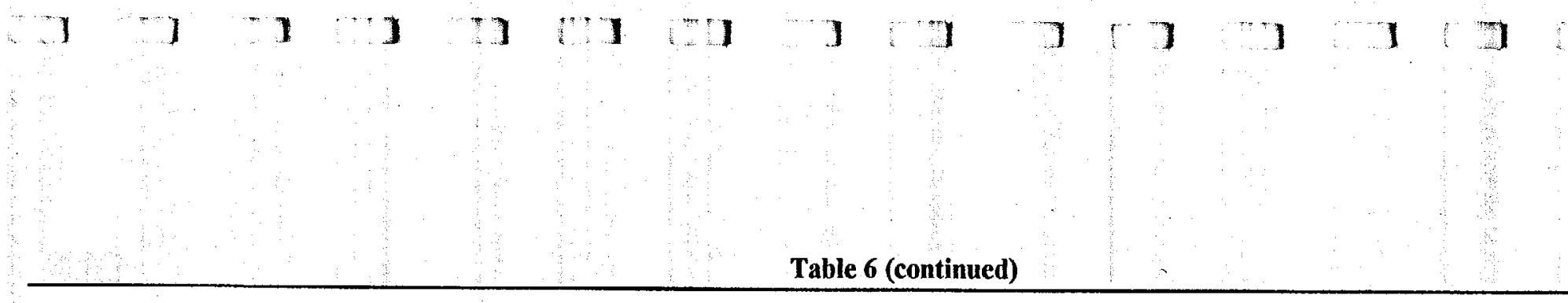

\begin{tabular}{|c|c|c|}
\hline Arca No. ${ }^{a}$ & $\begin{array}{l}\text { Date of } \\
\text { survey }\end{array}$ & Survey results and/or comments ${ }^{b}$ \\
\hline 12 & $4-15-97$ & $\begin{array}{l}\text {-High bay room with } 30 \text {-ft ceilings. } \\
\text {-Floor and floor-wall interfaces contaminated. This will be removed to access subsurface contamination. } \\
\text {-Surveyed walls and overheads ( } 50-60 \% \text { of overheads). } \\
\text { - Hot spot above guidelines on lower horizontal surface of upper I-beam (overheads on west wall). } \\
\text { - Ledge on the west wall with several areas on upper horizontal surfaces above guidelines. Will be } \\
\text { demolished with the floor. (Removed.) }\end{array}$ \\
\hline
\end{tabular}

Date released as below guidelines ${ }^{c}$

$12 \& 13$ 7-15-97

$12 \& 13$ 7-16-97

$12 \& 13$
-Gamma scan, 4 biased soil sampling locations identified.

-Beta-gamma scan of edges of footers.

-Began taking soil samples.

-Highest count rate where can access underside of concrete; maximum $37,000 \mathrm{dpm} / 100 \mathrm{~cm}^{2}$ at location J+0.5, 1 .

-Several spots along west knee wall $5000-10,000 \mathrm{dpm} / 100 \mathrm{~cm}^{2}$. (Approved as above-guideline area. ${ }^{{ }^{2}}$ ) -Southwest corner, concrete at wall-floor interface $25,000 \mathrm{dpm} / 100 \mathrm{~cm}^{2}$. (Removed) - One area soil contamination identified and remediated by IDM.
Surface above $1 \mathrm{ft}$ of floor-wall

interface 5-5-97
Area $12 \& 13$

subsurface \& remaining floor 7-30-97;

Area 12 10-27-97 


\begin{tabular}{|c|c|c|c|}
\hline Area No. ${ }^{a}$ & $\begin{array}{l}\text { Date of } \\
\text { survey }\end{array}$ & Survey results and/or comments ${ }^{b}$ & $\begin{array}{l}\text { Date released as } \\
\text { below guidelines }\end{array}$ \\
\hline 13 & $\begin{array}{l}3-4-96 \\
3-5-96\end{array}$ & 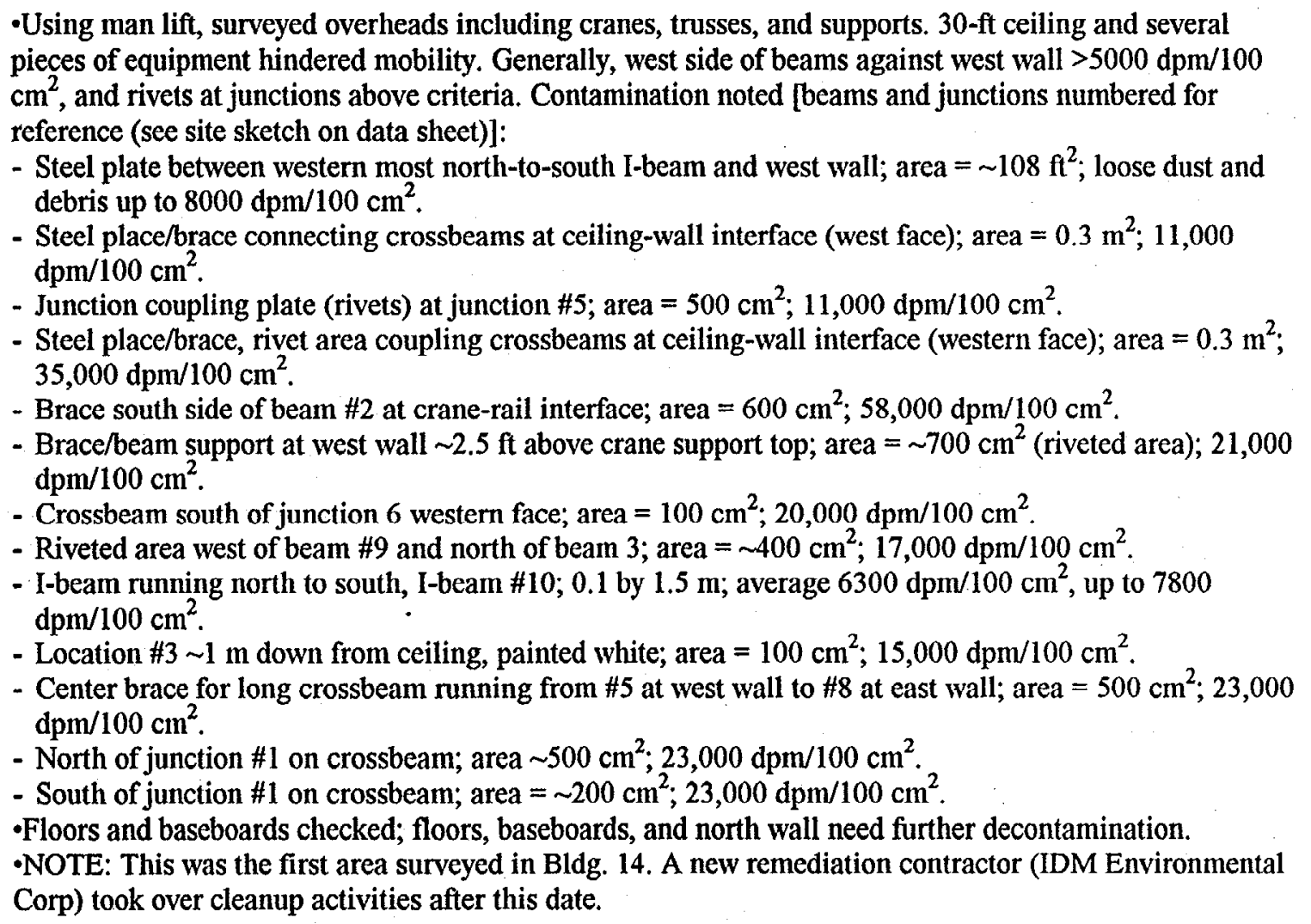 & \\
\hline 13 & 4-16-97 & $\begin{array}{l}\text {-Surveyed walls and overheads ( } 50-60 \% \text { overheads). } \\
\text { - Crain rail approved for above-guideline area. }{ }^{d} \\
\text {-Checked floors and floor-wall interfaces. }\end{array}$ & $\begin{array}{l}7-30-97 \\
7-7-98\end{array}$ \\
\hline
\end{tabular}




\begin{tabular}{|c|c|c|c|}
\hline Area No. ${ }^{a}$ & $\begin{array}{l}\text { Date of } \\
\text { survey }\end{array}$ & Survey results and/or comments ${ }^{b}$ & $\begin{array}{l}\text { Date released as } \\
\text { below guidelines }\end{array}$ \\
\hline $14 \mathrm{~N}$ & $1-13-98$ & $\begin{array}{l}\text {-Began survey of overheads. Generally } 600-1800 \mathrm{dpm} / 100 \mathrm{~cm}^{2} \text {. } \\
\text { - Spot on I-beam (at D, 2) } 17,000 \mathrm{dpm} / 100 \mathrm{~cm}^{2} \text {. See smear VT72. } \\
\text { - Spot on cross member (at } \mathrm{H}, 9) 17,000 \mathrm{dpm} / 100 \mathrm{~cm}^{2} \text {. See smear VT71. } \\
\left.\text { - Small spot } ~ 20 \mathrm{~cm}^{2} \text { (at } \mathrm{C}, 14\right) 14,000 \mathrm{dpm} / 100 \mathrm{~cm}^{2} \text {. Will average. See smear VT70. }\end{array}$ & \\
\hline $14 \mathrm{~N}$ & $1-14-98$ & $\begin{array}{l}\text { - Overheads scanned. } \\
\text {-Lower portion of walls scanned. }\end{array}$ & \\
\hline $14 \mathrm{~N}$ & $1-15-98$ & $\begin{array}{l}\text {-Completed survey of } \sim 50 \% \text { of overheads concentrating on probable areas of contamination (i.e., } \\
\text { horizontal surfaces, bolts, cross members). } \\
\text {-Surveyed bottom } 3 \text { meters of walls }(\sim 30 \%) \text {, } \\
\text { - Walls range from } 300-1800 \mathrm{dpm} / 100 \mathrm{~cm}^{2} \text { with an area } \sim 2600 \mathrm{dpm} / 100 \mathrm{~cm}^{2} \text {. }\end{array}$ & $\begin{array}{l}\text { All surfaces } \\
\text { above } 6 \text { in. from } \\
\text { floor-wall } \\
\text { interface } 1-30-98\end{array}$ \\
\hline $14 S W$ & $1-16-98$ & $\begin{array}{l}\text {-Surveyed lower areas to } \sim 10 \mathrm{ft} \text { up wall }\left(600-1800 \mathrm{dpm} / 100 \mathrm{~cm}^{2}\right) \text {. } \\
\text {-Anchor bolts } 2700-3300 \mathrm{dpm} / 100 \mathrm{~cm}^{2} \text {. }\end{array}$ & \\
\hline $\begin{array}{l}14 \mathrm{~N} \\
14 S W\end{array}$ & $3-4-98$ & $\begin{array}{l}\text {-Surveyed soil and lower portion of wall this trip. } \\
\text {-Several areas along the knee wall (concrete surface and soil underneath) above criteria. These have also } \\
\text { been identified by IDM and are to be included in the hazard assessment. } \\
\text {-Soil scanned with Nal detector; } 3 \text { biased soil sample locations identified. } \\
\text { - Collected } 3 \text { biased soil samples and } 15 \text { systematic samples. } \\
\text { - Scanned lower portion of wall (up to } \sim 10 \mathrm{ft} \text { ). } \\
\text { - Conferred with IDM (remediation contractor). Elevated soil on } 14 \mathrm{~N}-14 \mathrm{SW} \text { boundary to be removed } \\
\text { overnight. Area will be ready for verification tomorrow. }\end{array}$ & $\begin{array}{l}14 \mathrm{~N} \text { and } 14 \mathrm{SW} \\
\text { subsurface and } \\
\text { remaining floor } \\
3-16-98\end{array}$ \\
\hline $14 S W$ & $3-4-98$ & $\begin{array}{l}\text { - Contaminated concrete ledge } 20 \mathrm{ft} \text { up bordering } 14 \mathrm{SE} \text { (area with } 55 \text {-ft ceiling) will be removed with } \\
\text { decon of 14SE. }\end{array}$ & \\
\hline $\begin{array}{l}14 \mathrm{SE} \\
14 \mathrm{SW}\end{array}$ & $3-5-98$ & -Completed surface surveys and soil sampling. & 14SE 6-10-98 \\
\hline $14 \mathrm{SW}$ & $5-4-98$ & $\begin{array}{l}\text {-Overheads (not ceiling) } 0-1200 \mathrm{dpm} / 100 \mathrm{~cm}^{2} \text {. } \\
\text { - NW corner (overheads) on a former window sill, crack in concrete } \sim 4000-7000 \mathrm{dpm} / 100 \mathrm{~cm}^{2} \text {. }\end{array}$ & \\
\hline
\end{tabular}




\begin{tabular}{|c|c|c|c|}
\hline Area No. ${ }^{a}$ & $\begin{array}{l}\text { Date of } \\
\text { survey }\end{array}$ & Survey results and/or comments ${ }^{b}$ & $\begin{array}{l}\text { Date released as } \\
\text { below guidelines }\end{array}$ \\
\hline $14 S W$ & $5-5-98$ & $\begin{array}{l}\text { - Overheads (I-beams and cross members) surveyed with lift. } \\
-~ \sim 50 \% \text { coverage. } \\
\text { - All areas clean except the lower horizontal surfaces of I-beams closest to the wall. Areas will be added to } \\
\text { hazard assessment. }\end{array}$ & $\begin{array}{l}\text { 14SW areas } 12 \mathrm{ft} \\
\text { above floor } \\
5-20-98 \text {; Ceilings } \\
6-10-98\end{array}$ \\
\hline $\begin{array}{l}14 \mathrm{SW} \\
14 \mathrm{SE}\end{array}$ & $6-1-98$ & $\begin{array}{l}\text {-Surveyed } \sim 35-40 \% \text { of ceiling ( } 55-\mathrm{ft} \text { ceiling in Area } 14 \mathrm{SW}) \text { using scissor lift, generally } \\
300-1800 \mathrm{dpm} / 100 \mathrm{~cm}^{2} \text {. } \\
\text { - Found one slightly elevated area }\left(2400-3000 \mathrm{dpm} / 100 \mathrm{~cm}^{2}\right) \text { and one significantly elevated area } \\
\left(3300-59,000 \mathrm{dpm} / 100 \mathrm{~cm}^{2}\right) \text { located on the uppermost I-beam on the N wall. Contamination covered } \\
\sim 10-15 \mathrm{ft} \text { on horizontal (lower) surface. IDM chiseled away the significantly contaminated area. }\end{array}$ & $\begin{array}{l}14 \mathrm{SW} \text { accessible } \\
\text { areas above } 6 \text { in. } \\
\text { from floor-wall } \\
\text { interface } 1-30-98\end{array}$ \\
\hline $14 \mathrm{SE}$ & $6-3-98$ & $\begin{array}{l}\text { - Overheads and walls. } \\
\text { - After climbing } 25-30 \mathrm{ft} \text { on scaffolding, decided unsafe. } \\
\text { - Reviewed IDMs post-remedial action survey data. }\end{array}$ & $\begin{array}{l}\text { 10-10-98; } \\
\text { Ceilings 10-23-98 }\end{array}$ \\
\hline $14 \mathrm{~S}$ & $7-8-98$ & $\begin{array}{l}\text { - Surveyed above the bridge cranc, } \sim 25 \% \text { of horizontal surfaces and cracks and crevices likely to contain } \\
\text { contamination. The following spots and small areas were noted. All are below guidelines. } \\
\text { - Spot in NW corner, } \sim 500 \mathrm{~cm}^{2}=12,000 \mathrm{dpm} / 100 \mathrm{~cm}^{2} \text {. } \\
\text { - NW corner, horizontal on I-beam, } \sim 4 \mathrm{ft} \times 10 \mathrm{~cm}=3300 \mathrm{dpm} / 100 \mathrm{~cm}^{2} \text {. } \\
\text { - Spot at NW wall }=1600 \mathrm{dpm} / 100 \mathrm{~cm}^{2} . \\
\text { - Spot at } N \text { center wall, }<100 \mathrm{~cm}^{2}=3800 \mathrm{dpm} / 100 \mathrm{~cm}^{2} \text { (smear showed no transferable contamination). } \\
\text { - Along N wall, generally } 900-2400 \mathrm{dpm} / 100 \mathrm{~cm}^{2} \text { (red brick). } \\
\text { - Spot at NE wall }=1600 \mathrm{dpm} / 100 \mathrm{~cm}^{2} . \\
\text { - W ceiling vent }=\text { background. } \\
\text { - E ceiling vent }=900-2100 \mathrm{dpm} / 100 \mathrm{~cm}^{2} . \\
\text { - SE, } 2 \text {-ft } \times 4-\mathrm{ft} \text { area }=5000 \mathrm{dpm} / 100 \mathrm{~cm}^{2} . \\
\text { - SE, area } 18 \text { in. } \times 3 \mathrm{in} .=10,000-15,000 \mathrm{dpm} / 100 \mathrm{~cm}^{2} \text { (smear showed no transferable contamination). }\end{array}$ & \\
\hline 14SE & $7-16-98$ & $\begin{array}{l}\text { - Gamma scan of floor and subsurface }(11-13 \mu \mathrm{R} / \mathrm{h}) \text {. } \\
\text {-Systematic samples VS183-VS187 and biased sample VB45 }(13 \mu \mathrm{R} / \mathrm{h}) \text { collected this date. } \\
\text { - Remaining floor scanned with beta-gamma pancake detector }\left(300-2400 \mathrm{dpm} / 100 \mathrm{~cm}^{2}\right) \text {. } \\
\text {-One area above guidelines. This area to be chipped and IDM to take additional HP data. }\end{array}$ & \\
\hline 14 & & -Soil samples cleared & $10-23-98$ \\
\hline
\end{tabular}




\section{Table 6 (continued)}

\begin{tabular}{|c|c|c|c|}
\hline Area No. ${ }^{a}$ & $\begin{array}{l}\text { Date of } \\
\text { survey }\end{array}$ & Survey results and/or comments ${ }^{b}$ & $\begin{array}{l}\text { Date released as } \\
\text { below guidelines }\end{array}$ \\
\hline 15 & $11-10-97$ & $\begin{array}{l}\text {-Gamma scan } 10-13 \mu \mathrm{R} / \mathrm{h}, 14 \mu \mathrm{R} / \mathrm{h} \text { in corner geometry. } \\
\text {-Beta-gamma scan of floor-wall interfaces; small spot } 2500 \mathrm{dpm} / 100 \mathrm{~cm}^{2} \text {; No contamination above } \\
\text { guidelines. } \\
\text {-Surveyed all accessible floor areas with floor monitor; one area } 15,000 \mathrm{dpm} / 100 \mathrm{~cm}^{2} \text {; remediated by IDM. } \\
\text { - } 33 \% \text { of overheads surveyed; } 600-3300 \mathrm{dpm} / 100 \mathrm{~cm}^{2} \text {. } \\
\text {-Began wall scan. }\end{array}$ & . \\
\hline 15 & $11-11-97$ & $\begin{array}{l}\text { - Continued survey of overheads and walls. } \\
\text {-Surveyed pit in southern end of area. } \\
\text { - Released 11-12-97 }\end{array}$ & $12-12-97$ \\
\hline $15 \mathrm{~A}$ & $11-11-97$ & $\begin{array}{l}\text { - Contamination on column adjacent to Area } 15\left(10,000-15,000 \mathrm{dpm} / 100 \mathrm{~cm}^{2}\right) \text { covering area of } \sim 1 / 2 \mathrm{~m}^{2} \text {. } \\
\text {-Cleaned up by IDM. Cleared } 11-12-98 \text {. } \\
\text {-Completed walls. } \\
\text { - Took smears and alpha measurements }\end{array}$ & \\
\hline $\begin{array}{l}15 \mathrm{~A} \\
15 \mathrm{~B} 1 \\
15 \mathrm{~B}\end{array}$ & $11-12-97$ & -Need IDM data on overhead. & \\
\hline $15 \mathrm{~A}$ & $11-12-97$ & -Need IDM data on drain and pit. & \\
\hline $15 \mathrm{~A}$ & $11-12-97$ & -Completed overhead scan. & \\
\hline $15 \mathrm{~B}-1$ & $11-12-97$ & One spot $15,000 \mathrm{dpm} / 100 \mathrm{~cm}^{2}$. Cleaned up by IDM. & \\
\hline 15B-1 & $11-12-97$ & $\begin{array}{l}\text { - Checked drain near 15B-1. No contamination detected. } \\
\text {-Finished overheads. Overhead area cleared. }\end{array}$ & \\
\hline $\begin{array}{l}15 \mathrm{~A}, 15 \mathrm{~B} \\
15 \mathrm{~B}-1\end{array}$ & & -Interior surfaces and subsurface cleared & $\begin{array}{l}\text { Interior surfaces } \\
\text { and subsurfaces } \\
\text { cleared 12-22-97 }\end{array}$ \\
\hline
\end{tabular}


Table 6 (continued)

\begin{tabular}{|c|c|c|c|}
\hline Area No. ${ }^{a}$ & $\begin{array}{l}\text { Date of } \\
\text { survey }\end{array}$ & Survey results and/or comments ${ }^{b}$ & $\begin{array}{l}\text { Date released as } \\
\text { below guidelines }\end{array}$ \\
\hline $20 \mathrm{~A}$ & $7-18-96$ & $\begin{array}{l}\text {-Survey of remaining concrete floor and exposed subsurface area. Area cluttered by equipment, tools, and } \\
\text { storage shelves. Areas of note: } \\
\text { - } 2 \text {-ft by } 15 \text {-ft area on concrete floor at base of north wall with } 4 \text { to } 6 \text { spots }>15,000 \mathrm{dpm} / 100 \mathrm{~cm}^{2} \text {. } \\
\text { Recommend remediation. } \\
\text { - Subsurface area in southeast corner } 18 \mu \mathrm{R} / \mathrm{h} ; 14,000 \mathrm{dpm} / 100 \mathrm{~cm}^{2} \text { appears to continue north and east of } \\
\text { corner. } \\
\text { - Subsurface trench generally } 3300-5100 \mathrm{dpm} / 100 \mathrm{~cm}^{2} \text {. } \\
\text { - Plastic cover on pipe in trench } 15,000 \mathrm{dpm} / 100 \mathrm{~cm}^{2} \text {. } \\
\text { - } 0 \mathrm{~N}, 7 \mathrm{E} \text { vertical pipe, } \sim 3 \text { in., } 16,000 \mathrm{dpm} / 100 \mathrm{~cm}^{2} \text {. }\end{array}$ & $7-7-98$ \\
\hline 20A East & $\begin{array}{l}10-28-96 \\
10-29-96\end{array}$ & -No elevated areas. & $\begin{array}{l}12-30-96 \\
7-7-98\end{array}$ \\
\hline 20A West & & - Review of IDM data. & $1-30-98$ \\
\hline $\begin{array}{l}20 \mathrm{~B} \\
20 \mathrm{~B}-1\end{array}$ & $\begin{array}{l}10-28-96 \\
10-29-96\end{array}$ & -Surveyed $\sim 50 \%$ floor and wall surfaces. No elevated areas. & $\begin{array}{l}12-30-96 \\
7-7-98\end{array}$ \\
\hline $20 \mathrm{C}$ & $\begin{array}{l}10-28-96 \\
10-29-96\end{array}$ & -No elevated areas. & $\begin{array}{l}12-20-96 \\
7-7-98\end{array}$ \\
\hline 21 & $2-5-98$ & $\begin{array}{l}\text { - Obtained background information on activities conducted in the } 2 \text { sumps. Toured area. } \\
\text {-Sumps removed and excavated down to } 12-15 \mathrm{ft} \text {. } \\
\text { - Reviewed data from IDM. } \\
\text {-IDM split soil samples sent to ORNL for analysis. }\end{array}$ & $\begin{array}{l}7-7-98 \\
9-21-98\end{array}$ \\
\hline
\end{tabular}




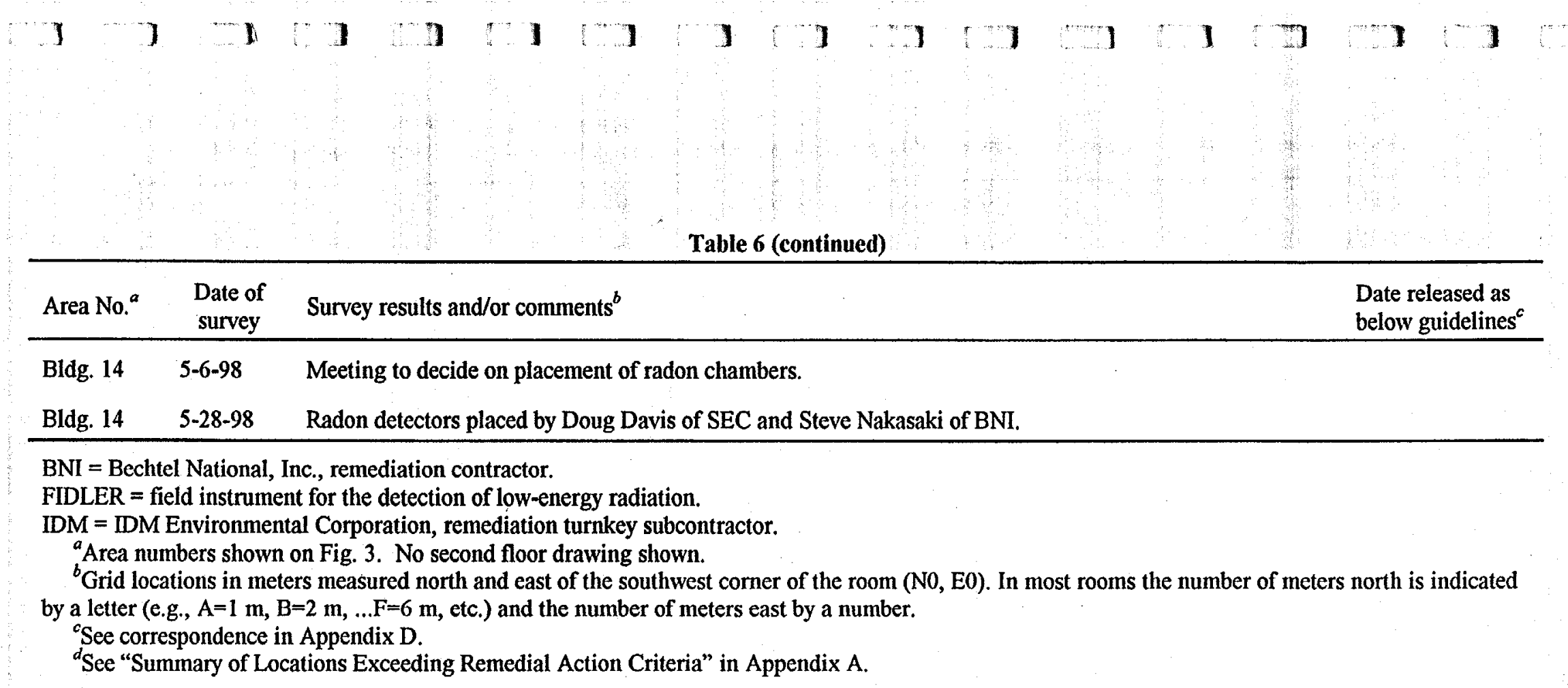


Table 7. Results of radon measurements in indoor air at Building 14, former Linde Uranium Refinery, Tonawanda, New York

\begin{tabular}{|c|c|c|c|c|c|c|}
\hline $\begin{array}{l}\text { Location in Building } 14 \\
\text { (see Fig. 5) }\end{array}$ & $\begin{array}{l}\text { Electret serial } \\
\text { number }\end{array}$ & Start date & Stop date & $\begin{array}{c}\text { Total time } \\
\text { hours (days) }\end{array}$ & $\begin{array}{l}\text { Radon concentration } \\
(\mathrm{pCi} / \mathrm{L})\end{array}$ & $\begin{array}{l}\text { Radon concentration }^{a} \\
\text { (WL) }\end{array}$ \\
\hline Areas 2 and 3 & SR5034 & $5-28-98$ & $6-29-98$ & $775.9(\sim 32)$ & 1.1 & 0.0055 \\
\hline Area 4B & SR5119 & $5-28-98$ & $6-29-98$ & $775.8(\sim 32)$ & 1.5 & 0.0075 \\
\hline First floor offices, $5 \mathrm{~A}$ & SO6225 & $5-28-98$ & $6-29-98$ & $775.4(\sim 32)$ & 0.7 & 0.0035 \\
\hline First floor offices, 5A & & $5-28-98$ & $6-29-98$ & $768.2(\sim 32)$ & 0.5 & 0.0025 \\
\hline Frist floor offices, 5B & SR5026 & $5-28-98$ & $6-30-98$ & $792.9(\sim 33)$ & 0.4 & 0.002 \\
\hline First floor offices, $5 \mathrm{C} 2$ & SO6122 & $5-28-98$ & $6-29-98$ & $775.5(\sim 32)$ & 1.2 & 0.006 \\
\hline First floor offices, $5 \mathrm{C} 3$ & SO6152 & $5-28-98$ & $6-29-98$ & $775.5(-32)$ & 0.6 & 0.003 \\
\hline Small Hallway & SR5168 & $5-28-98$ & $6-29-98$ & $775.9(\sim 32)$ & 0.5 & 0.0025 \\
\hline Area 8 & SR5182 & $5-28-98$ & $6-29-98$ & $776.1(\sim 32)$ & 1.2 & 0.006 \\
\hline Area 9 & SO6149 & $8-26-98$ & $9-28-98$ & $787.3(\sim 33)$ & 1.1 & 0.0055 \\
\hline Area 9 (duplicate) & SO6275 & $8-26-98$ & $9-28-98$ & $787.3(\sim 33)$ & 0.8 & 0.004 \\
\hline Corridor & $\mathrm{SO} 6323$ & $8-26-98$ & $9-28-98$ & $787.1(\sim 33)$ & 1.2 & 0.006 \\
\hline Area 13 & SR5233 & $5-28-98$ & $6-29-98$ & $776.2(\sim 32)$ & 0.9 & 0.0045 \\
\hline Area 13 (duplicate) & SR5199 & $5-28-98$ & $6-29-98$ & $775.2(\sim 32)$ & 0.8 & 0.004 \\
\hline Area 14-North & SO6157 & $8-26-98$ & $9-28-98$ & $787.2(\sim 33)$ & 0.6 & 0.003 \\
\hline Area 14-South & $\mathrm{SO6245}$ & $9-10-98$ & $9-28-98$ & $427.4(\sim 18)$ & 1.6 & 0.008 \\
\hline Area 15 & SO6126 & $5-28-98$ & $6-29-98$ & $775.8(\sim 32)$ & 0.7 & 0.0035 \\
\hline Area 20A-East & SR5229 & $5-28-98$ & $6-29-98$ & $775.7(\sim 32)$ & 1.2 & 0.006 \\
\hline Area 20A-West & SR5013 & $5-28-98$ & $6-29-98$ & $775.8(\sim 32)$ & 0.7 & 0.0035 \\
\hline Area 20B & SR5225 & $5-28-98$ & $6-29-98$ & $775.8(\sim 32)$ & 0.5 & 0.0025 \\
\hline
\end{tabular}

${ }^{a}$ Working level (WL) measurements were not performed. The listed values were estimated using the assumption that the concentration of radon progeny in room air was equal to $50 \%$ of the measured ${ }^{222} \mathrm{Rn}$ concentration. 


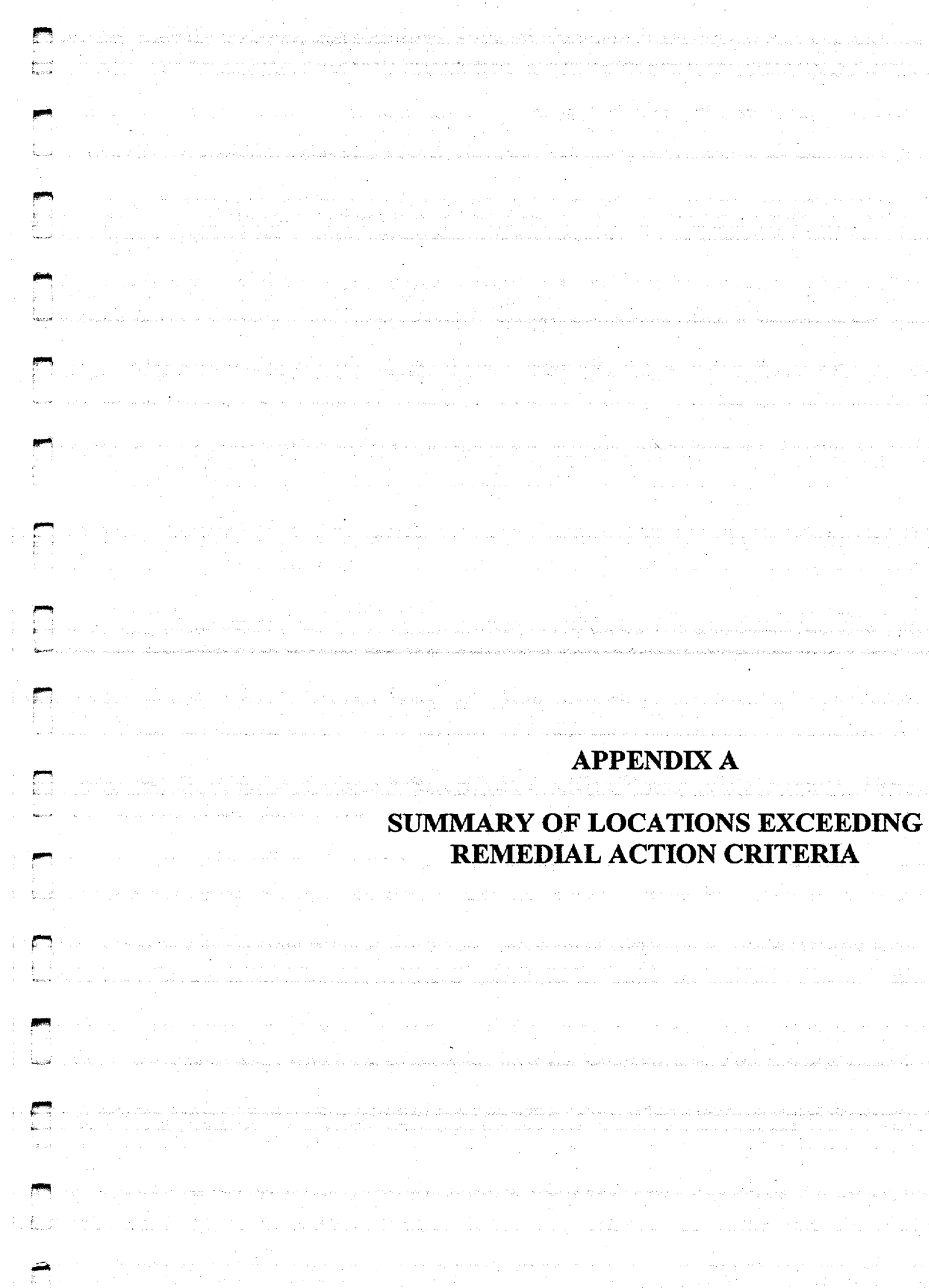


A number of locations were identified where residual contamination exceeding the remedial action criteria remained after decontamination efforts. These locations occur in Areas 9, 12, 13, 14 North, 14 South, 15, 20A East, and 21. Contamination exceeding the criteria was left in place only after all best efforts at decontamination were made and the criteria for supplemental limits were carefully evaluated. These locations fall into several categories. The first category includes areas of soils underlying building walls in Areas 12,13 , 14 North, and 14 South where further removal would undermine the walls and place the structural integrity of the building at risk. A second type, which occurred at five locations in Area 14 South, is on the sill of a beam adjacent to walls where limited access by remediation equipment prevented complete decontamination. Similarly, on the crane rails in Areas 12,13, and 14 North, restricted access prevented full decontamination around bolt heads. Four floor locations in Areas 9, 14 South, and 15 were inaccessible because of the presence of large equipment. Based on data from surrounding floor measurements, these locations were estimated to exceed guidelines. Interior wall contamination exceeding remedial action criteria in the south wall of Area 14 South was discovered. Several subsurface drainpipes in Area 9, the Area 12 stairwell sump, Area 20A East, and the existing in-bed drainline system that was left in place contained contamination exceeding remedial action criteria. This determination was based on survey measurements obtained at locations where drainlines were exposed during remediation of sumps or pipes. Portions of the drainline were removed during the remedial action, but most of the potentially contaminated drainline system remain in place. (Excerpt from Executive Summary in Post-Remedial Action Report for Building 14 at the Linde Site, Tonawanda, New York, June 1999.) 
Table 5-1 (Page 1 of 5)

Summary of Locations Exceeding Remedial Action Criteria

\begin{tabular}{|c|c|c|}
\hline $\begin{array}{l}\text { Location No. } \\
\text { (Area) }\end{array}$ & Description $^{\mathrm{a}}$ & $\begin{array}{c}\text { Rationale for Residual } \\
\text { Contamination Exceeding } \\
\text { Criteria }\end{array}$ \\
\hline $\begin{array}{l}\text { LEC-B14-1 } \\
\text { (Building } 14 \\
\text { subsurface) }\end{array}$ & $\begin{array}{l}\text { IN-BED DRAINLINES. Description: Includes an estimated } 543 \text { linear feet of the trench drainline system } \\
\text { left in place ( } 191 \text { feet were removed) beneath Building } 14 \text {. The drainlines were delineated at seven locations } \\
\text { where exposed and limited remedial actions were taken on certain sections. Drainlines are estimated to be up } \\
\text { to } 8 \text { feet below grade and pass underneath load-bearing walls. Remedial Actions: Exposed sections of } \\
\text { drainline were removed or plugged in the Area } 12 \text { stairwell, the Corridor, Area } 9 \text { and the Large Hallway. } \\
\text { Contamination Levels: Direct } \beta \gamma \text { activity measurements ranged from } 5,480 \text { to } 160,000 \mathrm{dpm} / 100 \mathrm{~cm}^{2} \text { in the } \\
\text { accessible sections. Data References: IDM Surveys } 217,373,403,687,747,1040 \text {, and } 1670 \text {. IDM Sample } \\
268 \text {. }\end{array}$ & $\begin{array}{l}\text { Contaminated drainlines are } \\
\text { inaccessible and remediation not } \\
\text { cost-effective. }\end{array}$ \\
\hline $\begin{array}{l}\text { LEC-9-1 } \\
\text { LEC-9-2 } \\
\text { (Area 9) }\end{array}$ & $\begin{array}{l}\text { FLOOR UNDERNEATH FUME HOODS. Description: Floor under two banks of fume hoods near the } \\
\text { east wall. Each fume hood covers an area of about } 2 \mathrm{~m} \mathrm{by} 5 \mathrm{~m} \text { or about } 107 \text { square feet for a total of } 214 \\
\text { square feet. Remedial Actions: Floor inaccessible except in the northeast corner, which were decontaminated } \\
\text { and a drain removed and plugged. Contamination Levels: Adjacent concrete direct } \beta \gamma \text { activity }-15,620 \text { to } \\
19,015 \mathrm{dpm} / 100 \mathrm{~cm}^{2} \text { - was assumed representative of fume hood floor. Data References: IDM Surveys } \\
1032 \text { and } 1033 \text {. }\end{array}$ & $\begin{array}{l}\text { Floor inaccessible without } \\
\text { removal of fume hoods. } \\
\text { Estimated cost was } \sim \$ 250,000 \text {. }\end{array}$ \\
\hline $\begin{array}{l}\text { LEC-9-3 } \\
\text { (Area 9) }\end{array}$ & $\begin{array}{l}\text { DRAINPIPE. Description: A 10-foot-long section of a 4-inch-diameter cast iron drainpipe was left in place. } \\
\text { Pipe was encased in concrete block which went underneath the south wall of Area } 9 \text {. Pipe appears to be } \\
\text { MED-era drainpipe from Linde drawing A63726. Remedial Actions: The remainder of the drainpipe and } \\
\text { surrounding soil was removed up to the eastern wall. Contamination Levels: Direct } \beta \gamma \text { activity was } 21,000 \\
\text { to } 73,000 \mathrm{dpm} / 100 \mathrm{~cm}^{2} \text { at west end of drainpipe. Data References: IDM Survey } 1500 \text {. }\end{array}$ & $\begin{array}{l}\text { Removal of remaining drainpipe } \\
\text { and subgrade concrete block } \\
\text { would affect the structural } \\
\text { integrity of the south wall. }\end{array}$ \\
\hline $\begin{array}{l}\text { LEC-9-4 } \\
\text { (Area 9) }\end{array}$ & $\begin{array}{l}\text { DRAINPIPE. Description: A drainpipe is present underneath the fume hood designated at LEC-9-1. The } \\
\text { pipe is presumed contaminated based on the connecting contaminated floor drain removed from underneath } \\
\text { the northeast corner of this fume hood. The direction of the pipe nun is believed to bc north-to-south but } \\
\text { could not be confirmed. Remedial Actions: The floor underneath the northeast corner of the fume hood, the } \\
\text { only part accessible, was decontaminated and a floor drain removed as part of the floor decontamination. The } \\
\text { drain passed through an elbow beyond which the drainpipe could not be observed. No further remedial action } \\
\text { was taken on the elbow or drainpipe. Contamination Levels: A direct } \beta \gamma \text { measurement taken on the floor } \\
\text { drain showed activity at } 21,000 \mathrm{dpm} / 100 \mathrm{~cm}^{2} \text {. Data References: IDM Survey } 1670 \text {. }\end{array}$ & $\begin{array}{l}\text { Removal of the drainpipe would } \\
\text { require removal of the fume } \\
\text { hoods which has already been } \\
\text { deemed not cost-effective. }\end{array}$ \\
\hline
\end{tabular}

1

1

1

1




\begin{tabular}{|c|c|c|}
\hline $\begin{array}{c}\text { Location No. } \\
\text { (Area) }\end{array}$ & Description & $\begin{array}{c}\text { Rationale for Residual } \\
\text { Contamination Exceeding } \\
\text { Criteria }\end{array}$ \\
\hline $\begin{array}{l}\text { LEC-12/13-1 } \\
\text { LEC-12/13-2 } \\
\text { (Areas 12/13) }\end{array}$ & $\begin{array}{l}\text { SOIL UNDERNEATH WEST WALL. Description: Soil was left underneath the horizontal concrete slab } \\
\text { which encases the electrical conduit down to the base of the excavation and back to the concrete footer } \\
\text { supporting the west wall. LEC-12/13-1 is estimated to be } 18 \text { feet long by } 1 \text { foot wide by } 1.5 \text { feet deep for a } \\
\text { total volume of } 27 \text { cubic feet. LEC-12/13-2 is estimated to be } 24.5 \text { feet long by } 1 \text { foot wide by } 1.5 \text { feet deep } \\
\text { for a total volume of } 37 \text { cubic feet. Remedial Action: The adjacent soil in Area } 12 \text { was excavated to a depth } \\
\text { of } 4 \text { feet. Soil was excavated in spots beneath the horizontal slab. Contamination Levels: Soil samples } \\
\text { collected underneath the horizontal slab had total uranium activities ranging from } 35.83 \text { to } 17,910 \mathrm{pCi} / \mathrm{g} \text {. } \\
\text { Data References: IDM Survey } 3001 \text {. IDM Samples } 344,346,352,357,365,417 \text {, and } 429 \text {. }\end{array}$ & $\begin{array}{l}\text { Removal would compromise the } \\
\text { structural integrity of the } \\
\text { building. }\end{array}$ \\
\hline $\begin{array}{l}\text { LEC-12/13-3 } \\
\text { LEC-12/13-4 } \\
\text { (Areas 12/13) }\end{array}$ & $\begin{array}{l}\text { SOIL UNDERNEATH CENTER WALL. Description: Soil was left underneath the center wall dividing } \\
\text { Areas } 12 \text { and } 13 \text { extending out } 1 \text { foot from each side. LEC-12/13-3 is estimated to be } 16 \text { feet long by } 2.5 \text { feet } \\
\text { wide by } 1 \text { foot deep for a total volume of } 41 \text { cubic feet. LEC-12/13-4 is estimated to have the same } \\
\text { dimensions for a total volume of } 41 \text { cubic feet. Remedial Action: Soil was excavated on both sides of the } \\
\text { walls up to within a foot of either side. The soil on the Area } 12 \text { side was removed to a depth of about } 4 \text { feet } \\
\text { and on the Area } 13 \text { side to a depth of about } 3 \text { feet. Contamination Levels: Eight samples were collected from } \\
\text { the sides of the walls containing total uranium activities of } 8 \text { to } 4,297 \mathrm{pCi} / \mathrm{g} \text {. Data References: IDM Surveys } \\
3002 \text { and } 3003 \text {. IDM Samples } 338,345,350,354,355,356,359,364,366,411,412 \text { and } 418 \text {. }\end{array}$ & $\begin{array}{l}\text { Removal would compromise the } \\
\text { structural integrity of the } \\
\text { building. }\end{array}$ \\
\hline $\begin{array}{l}\text { LEC-12/13-5 } \\
\text { LEC-12/13-6 } \\
\text { (Areas 12/13) }\end{array}$ & $\begin{array}{l}\text { SOIL UNDERNEATH SOUTH WALL. Description: Soil was left underneath the south wall of Arcas } 12 \\
\text { and } 13 \text { extending out } 1 \text { foot from each side. LEC-12/13-5 is estimated to be } 41 \text { feet long by } 2 \text { feet wide by } \\
1 \text { foot deep for a total volume of } 82 \text { cubic feet. LEC-12/13-6 is estimated to be } 6.5 \text { feet long by } 2 \text { feet wide by } \\
1 \text { foot deep for a total volume of } 13 \text { cubic feet. Remedial Action: Soil was excavated up to within a foot of the } \\
\text { wall. The depth of excavation ranged from } 4 \text { feet in Area } 12 \text { to } 2 \text { feet in Area } 13 \text {. Contamination Levels: } \\
\text { Seven samples collected from the sides of the excavation contained total uranium concentrations of } 38 \text { to } \\
3,614 \mathrm{pCi} / \mathrm{g} \text {. Data References: IDM Surveys } 3004 \text { and } 3005 \text {. IDM Samples } 337,353,360,362,363,367 \text {, } \\
\text { and } 368 \text {. }\end{array}$ & $\begin{array}{l}\text { Removal would compromise the } \\
\text { structural integrity of the } \\
\text { building. }\end{array}$ \\
\hline $\begin{array}{l}\text { LEC-12/13-7 } \\
\text { (Areas 12/13) }\end{array}$ & $\begin{array}{l}\text { STAIRWELL SUMP NORTH DRAINLINE. Description: This drainline connects the sump at the base of } \\
\text { the stairwell to the trench in the utility tunnel to the north. The drainline is about ten feet long. Remedial } \\
\text { Action: The sump floor and walls were decontaminated and rebuilt. The drainline was not decontaminated. } \\
\text { Contamination Levels: Direct } \beta / \gamma \text { activity within the drainline ranged from } 9,600 \text { up to } 54,000 \mathrm{dpm} / 100 \mathrm{~cm}^{2} \text {. } \\
\text { A sample of scale material removed from the pipe contained total uranium at } 6,573 \mathrm{pCi} / \mathrm{g} \text {. Data References: } \\
\text { IDM Surveys } 810 \text { and } 1260 \text {. IDM Sample } 790 \text {. }\end{array}$ & $\begin{array}{l}\text { Conventional decontamination } \\
\text { methods judged ineffective. } \\
\text { Alternative removal methods } \\
\text { destructive or not cost-effective. } \\
\text { The potential for } \\
\text { recontamination from utility } \\
\text { tunnel should be addressed } \\
\text { before pipe decontamination is } \\
\text { considered. }\end{array}$ \\
\hline
\end{tabular}




\begin{tabular}{|c|c|c|}
\hline $\begin{array}{l}\text { Location No. } \\
\text { (Area) }\end{array}$ & Description ${ }^{\mathrm{a}}$ & $\begin{array}{c}\text { Rationale for Residual } \\
\text { Contamination Exceeding } \\
\text { Criteria }\end{array}$ \\
\hline $\begin{array}{l}\text { LEC-12/13-8 } \\
\text { LEC-12/13-9 } \\
\text { (Areas 12/13) }\end{array}$ & $\begin{array}{l}\text { CENTER CRANE RAILS. Description: Channel steel (cross members) connect center crane rails to each } \\
\text { other. Area surrounding bolts connecting cross members to the crane rails remains contaminated above } \\
\text { surface guidelines following decontamination. Total area estimated at } 17 \mathrm{ft}^{2} \text {. Remedial Action: Crane rails } \\
\text { and cross members at these locations were decontaminated repeatedly by sponge blasting. Confined space } \\
\text { ( } 2 \text { to } 3 \text { inches clearance) prevented decontamination to below guidelines around bolts. Contamination Levels: } \\
\text { Post-decontamination dircet } \beta \gamma \text { activity was } 8,300 \text { to } 19,000 \mathrm{dpm} / 100 \mathrm{~cm}^{2} \text {. Data References: IDM Survey } \\
404 \text {. }\end{array}$ & $\begin{array}{l}\text { Removal of contamination is not } \\
\text { feasible due to inaccessibility } \\
\text { with conventional } \\
\text { decontamination equipment. }\end{array}$ \\
\hline $\begin{array}{l}\text { LEC-14N-1 } \\
\text { LEC-14N-2 } \\
\text { LEC-14N-3 } \\
\text { (Area } 14 \mathrm{~N} \text { ) }\end{array}$ & $\begin{array}{l}\text { WEST KNEE WALL: Description: Concrete on knee wall below grade remains contaminated above } \\
\text { criteria. LEC-14N-1 -2 and }-3 \text { total } 5.5,1.3 \text {, and } 0.5 \text { square feet, respectively. Remedial Actions: The knee } \\
\text { wall was aggressively decontaminated with only these locations remaining above surface guidelines. } \\
\text { Contamination Levels: Direct } \beta \gamma \text { activity after decontamination was } 600 \text { to } 19,062 \mathrm{dpm} / 100 \mathrm{~cm}^{2} \text {. Data } \\
\text { References: IDM Survey } 1267 \text {. }\end{array}$ & $\begin{array}{l}\text { Further decontamination or } \\
\text { removal of the knee wall would } \\
\text { affect the structural integrity of } \\
\text { the building. }\end{array}$ \\
\hline $\begin{array}{l}\text { LEC-14N-4 } \\
\text { LEC-14N-5 } \\
\text { LEC-14N-6 } \\
\text { (Area 14N) }\end{array}$ & $\begin{array}{l}\text { SOIL UNDERNEATH NORTH WALL: Description: Soil underlying wall left in place to support wall. } \\
\text { LEC-14N-4 comprises } 12 \mathrm{ft}^{3} \text { of ash } 4 \text { to } 8 \text { inches beneath slab. LEC-14N-5 contains } 12 \mathrm{ft}^{3} \text { of ash and } 12 \mathrm{ft}^{3} \text { of } \\
\text { clay soil } 4 \text { to } 20 \text { inches beneath slab. LEC-14N-6 contains } 9 \mathrm{ft}^{3} \text { of ash } 4 \text { to } 8 \text { inches beneath slab. Remedial } \\
\text { Actions: Concrete floor slab and underlying soil were excavated as close as practical to the wall. } \\
\text { Contamination Levels: LEC-14N-5 and }-6 \text { at } 247 \mathrm{pCi} / \mathrm{g} \text { total uranium (Sample 877). LEC-14N-4 measured } \\
\text { at } 14.9 \mathrm{pCi} / \mathrm{g} \text { total uranium (Sample } 876 \text { ), but contamination on Area } 12 \text { side of wall makes this a } \\
\text { supplemental limit location. Data References: IDM Samples } 876 \text { and } 877 \text {, IDM Survey } 1267 \text {. }\end{array}$ & $\begin{array}{l}\text { Further removal of the soil } \\
\text { would affect the structural } \\
\text { integrity of the building. }\end{array}$ \\
\hline $\begin{array}{l}\text { LEC-14N-7 } \\
\text { LEC-14N-8 } \\
\text { (Area 14N) }\end{array}$ & $\begin{array}{l}\text { CRANE RAIL CROSS MEMBERS: Description: Channel steel (cross members) connect center crane rails } \\
\text { to each other and east crane rail to wall. Area surrounding bolts connecting cross members to the crane rails } \\
\text { remains contaminated above surface guidelines following decontamination. Total area estimated at } 15 \mathrm{ft}^{2} \text {. } \\
\text { Remedial Actions: Crane rails and cross members at these locations were decontaminated repeatedly by } \\
\text { sponge blasting. Confined space ( } 2 \text { to } 3 \text { inches clearance) prevented decontamination to below guidelines } \\
\text { around bolts. Contamination Levels: Post-decontamination direct } \beta \gamma \text { activity was } 4,600 \text { to } 27,000 \mathrm{dpm} / \\
100 \mathrm{~cm}^{2} \text {. Data References: IDM Surveys } 1131 \text { and } 1139 \text {. }\end{array}$ & $\begin{array}{l}\text { Removal of contamination is not } \\
\text { feasible due to inaccessibility } \\
\text { with conventional } \\
\text { decontamination equipment. }\end{array}$ \\
\hline $\begin{array}{l}\text { LEC-14S-1 } \\
\text { LEC-14S-2 } \\
\text { (Area 14S) }\end{array}$ & $\begin{array}{l}\text { WEST WALL FOOTER AND SOIL. Description: LEC-14S-1 contains } 1 \mathrm{ft}^{3} \text { of soil beneath the knee wall } \\
\text { which exceeds criteria and } 2 \mathrm{ft}^{2} \text { of subgrade knee wall which exceeds guidelines. At LEC-14S-2, } 1 \mathrm{ft}^{2} \text { of knee } \\
\text { wall concrete exceeds guidelines. Remedial Actions: Soil was excavated as close to the knee wall as practical } \\
\text { and underneath it at some locations, and the concrete surface was aggressively decontaminated. } \\
\text { Contamination Levels: Soil at } 78.9 \text { to } 87.8 \mathrm{pCi} / \mathrm{g} \text {, knee wall at } 600 \text { to } 5,215 \mathrm{dpm} / 100 \mathrm{~cm}^{2} \text {. Data References: } \\
\text { IDM Samples } 878 \text { and } 879 \text {. IDM Survey } 1267 \text {. }\end{array}$ & $\begin{array}{l}\text { Further decontamination or } \\
\text { removal of the knee wall and soil } \\
\text { would affect the structural } \\
\text { integrity of the building. }\end{array}$ \\
\hline
\end{tabular}

1

1

1

1




\begin{tabular}{|c|c|c|}
\hline $\begin{array}{l}\text { Location No. } \\
\text { (Area) }\end{array}$ & Description & $\begin{array}{c}\text { Rationale for Residual } \\
\text { Contamination Exceeding } \\
\text { Criteria }\end{array}$ \\
\hline $\begin{array}{l}\text { LEC-14S-3 } \\
\text { (Area 14S) }\end{array}$ & $\begin{array}{l}\text { SOUTH WALL CONCRETE FOOTER/BRICK WALL INTERFACE. Description: Interface between } \\
\text { concrete south wall footer and the brick remains contaminated above surface guidelines. Extends about } 42 \\
\text { feet underneath the outermost brick, or two bricks, at the east end. Total area impacted about } 13 \mathrm{ft}^{2} \text {. } \\
\text { Remedial Actions: First two layers of inner two courses of brick removed from interior wall and footer } \\
\text { surface decontaminated. Contamination Levels: Pre-decontamination direct } \beta \text { yctivity of footer ranged from } \\
10,164 \mathrm{dpm} / 100 \mathrm{~cm}^{2} \text { to } 132,492 \mathrm{dpm} / 100 \mathrm{~cm}^{2} \text {. Assumed direct } \beta / \gamma \text { activity average is } 30,769 \mathrm{dpm} / 100 \mathrm{~cm}^{2} \text {. } \\
\text { Data References: IDM Survey } 1752 \text { (pre-remedial data) and } 1737 \text { (post-remedial data). }\end{array}$ & $\begin{array}{l}\text { Further removal of the brick wall } \\
\text { would affect the structural } \\
\text { integrity of the building. }\end{array}$ \\
\hline $\begin{array}{l}\text { LEC-14S-4 } \\
\text { LEC-14S-5 } \\
\text { LEC-14S-6 } \\
\text { LEC-14S-7 } \\
\text { LEC-14S-8 } \\
\text { (Area 14S) }\end{array}$ & $\begin{array}{l}\text { HORIZONTAL I-BEAMS (SOUTH, NORTH, AND EAST WALLS). Description: These are structural } \\
\text { steel beams which are adjacent to the walls in Area } 14 \text { South. Locations are on the upper surface of the lower } \\
\text { lip of each I-beam on the side of the beam closest to the wall. Access to this surface by decontamination } \\
\text { equipment was difficult, but the vertical and upper surfaces of the same side of the beams were successfully } \\
\text { decontaminated below criteria. Total area }=7.3 \mathrm{ft}^{2} \text { per I-beam and } 36.5 \mathrm{ft}^{2} \text { total. Remedial Actions: All } \\
\text { surfaces of the beams were decontaminated by sponge blasting. Repeated attempts were made to remove } \\
\text { contamination on these locations. Contamination Levels: Residual dircct } \beta \gamma \text { activity lcvcls rangcd } 3,000 \text { to } \\
36,000 \mathrm{dpm} / 100 \mathrm{~cm}^{2} \text { (south wall), } 5,000 \text { to } 10,000 \mathrm{dpm} / 100 \mathrm{~cm}^{2} \text { (east wall) and } 6,000 \text { to } 9,000 \mathrm{dpm} / 100 \mathrm{~cm}^{2} \\
\text { (north wall). Data References: IDM Surveys } 1347,1351,1354,1374 \text {, and } 1498 \text {. }\end{array}$ & $\begin{array}{l}\text { Removal of contamination is not } \\
\text { feasible due to inaccessibility } \\
\text { with conventional } \\
\text { decontamination equipment }\end{array}$ \\
\hline $\begin{array}{l}\text { LEC-14S-11 } \\
\text { (Area 14S) }\end{array}$ & $\begin{array}{l}\text { FLOOR UNDERNEATH COLUMN 1. Description: The floor underneath the concrete pad supporting } \\
\text { Column 1, at grid cell E-13, is inaccessible and is estimated to exceed surface guidelines. The size of the } \\
\text { affected floor area is approximately } 5 \text { feet by } 8 \text { feet for a total area of } 40 \text { square feet. Remedial Actions: The } \\
\text { floor areas around and up to the concrete pad were decontaminated or removed. Contamination Levels: The } \\
\text { estimated activity of this floor area is } 5,245 \text { dpm/100 } \mathrm{cm}^{2} \text {. Data References: Surveys are described in "Direct } \\
\text { Surface and Transferable Contamination Survey," BNI CCN No. D-28336 (1997). }\end{array}$ & $\begin{array}{l}\text { Decontamination is not cost- } \\
\text { effective as it would require } \\
\text { removal of process column. }\end{array}$ \\
\hline
\end{tabular}




\begin{tabular}{|c|c|c|}
\hline $\begin{array}{l}\text { Location No. } \\
\text { (Area) }\end{array}$ & Description $^{\mathrm{a}}$ & $\begin{array}{c}\text { Rationale for Residual } \\
\text { Contamination Exceeding } \\
\text { Criteria }\end{array}$ \\
\hline $\begin{array}{l}\text { LEC-15-1 } \\
\text { (Area 15) }\end{array}$ & $\begin{array}{l}\text { FLOOR UNDER TANK. Description: A large } 20 \text {-foot-diameter process tank located near the south end of } \\
\text { Area } 15 \text {. The tank rests on what is presumably the original concrete slab. Remedial Actions: Surrounding } \\
\text { floor was delineated and decontaminated where necessary. Contamination Levels: The floor underneath the } \\
\text { tank is believed to have levels of contamination similar to those found on the surrounding floor at direct } \beta y \\
\text { activity up to } 27,000 \mathrm{dpm} / 100 \mathrm{~cm}^{2} \text { with the highest levels present west of the tank. Data References: IDM } \\
\text { Survey } 355 \text {. }\end{array}$ & $\begin{array}{l}\text { Relocation of tank and two other } \\
\text { columns judged not } \\
\text { economically feasible at } \\
\$ 375,000 \text { and would have } \\
\text { impacted owner operations. }\end{array}$ \\
\hline $\begin{array}{l}\text { LEC-20AE-1 } \\
\text { (Area 20A } \\
\text { East) }\end{array}$ & $\begin{array}{l}\text { DRAINPIPE. Description: An abandoned drainpipe leading south from the center trench is present in the } \\
\text { western trench. Historical drawings suggest it is a 4-inch diameter MED-era pipe. Pipe appears to run out of } \\
\text { the building and likely leads to the sanitary sewer. Remedial Actions: The plug appeared to have been } \\
\text { capped to prevent flow and trench removed. No further remedial actions implemented on drainpipe. } \\
\text { Contamination Levels: The drainpipe was surveyed by TNU in } 1996 \text {. Measurements were taken up to } 22 \text { feet } \\
\text { from the trench with the maximum direct } \beta / \text { activity of } 41,094 \mathrm{dpm} / 100 \mathrm{~cm}^{2} \text { measured at } 12 \text { feet from the } \\
\text { trench. Data References: TNU Survey } 129 D T 032 \text {, sheets } 12900975 \text {.xls and } 12900976 . x 1 s \text {. }\end{array}$ & $\begin{array}{l}\text { Removal of pipe would require } \\
\text { excavation under north wall of } \\
\text { Room } 20 \mathrm{~B}-1 \text { and removal of } \\
\text { footer of the south wall of the } \\
\text { building, which would } \\
\text { compromise the structural } \\
\text { integrity of the building. }\end{array}$ \\
\hline $\begin{array}{c}\text { LEC-21-1 } \\
\text { (Area 21) }\end{array}$ & $\begin{array}{l}\text { SETTLING BASIN CONCRETE FLOOR BENEATH PIPES. Description: A former settling basin, used } \\
\text { during MED operations, was buried beneath the slab within the current footprint of the Butler Building. } \\
\text { Several water pipes ran through the basin and were supported in the west section by a tightly compacted rock } \\
\text { and gravel mixture with concrete encasing the pipes on the top. The rock covers an area of } 10 \text { feet by } 2 \text { feet } \\
\text { or a total of } 20 \mathrm{ft}^{2} \text {. Remedial Actions: The fill material within the settling basin was excavated and removed. } \\
\text { The concrete walls and floor of the basin were removed with the exception of the floor beneath the pipes and } \\
\text { rock supporting the pipes. Contamination Levels: The concrete surface of the settling basin floor adjacent to } \\
\text { the remaining rock which supports the pipes was surveyed with direct } \beta y \text { activity of } 26,000 \text { to } 39,000 \\
\mathrm{dpm} / 100 \mathrm{~cm}^{2} \text {. Data References: IDM Survey } 1185 \text {. }\end{array}$ & $\begin{array}{l}\text { Removal of concrete and rock } \\
\text { supporting water supply pipes } \\
\text { may risk damage to the pipes. }\end{array}$ \\
\hline
\end{tabular}

Note:

Additional details on the description and locations of each location exceeding the remedial action criteria can be obtained in the Section 4 text and summary figures of this report. 


\title{
SUMMARY REPORT \\ FOR THE \\ PROCESS PIPING RADIOLOGICAL INVESTIGATION \\ PRAXAIR BUILDING 14
}

\author{
RECEIVED \\ JAN 121998 \\ Prepared for \\ IDM ENVIRONMENTAL CORP. \\ SOUTH RIVER, NJ 08882 \\ SUBCONTRACT NO. 14501-129-SC-563
}

\section{BNI / FUSRAP NY REGION \\ TONAWANDA, NY}

Prepared by

ION TECHNOLOGY, INC. SARATOGA SPRINGS, NEW YORK 


\section{TABLE OF CONTENTS}

COVER PAGE

TABLE OF CONTENTS

1.0 Site and Project Information 2

1.1 Background Information

1.2 Historical Information

1.3 Scope of Project

2.0 Process Piping Radiological Investigation Approach

\subsection{Survey Methodology}

3.0 Independent Verification Contractor (IVC) 4

4.0 Radiological Survey Findings and Results 5

\subsection{External Surfaces}

4.2 Internal Surfaces

5.0 Summary

6.0 References

\section{ATTACHMENTS}

Attachment 1

Attachment 2

Attachment 3

Attachment 4

Attachment 5

Attachment 6
B14 Process Piping Investigation Procedure

Building 14 Plan View Area Map

Process Piping Radiological Surveys

HpGe Analytical Reports

Radiation Detection Instrumentation

Summary of B14 Process Piping LF 


\section{B-5}

\subsection{SITE AND PROJECT INFORMATION}

\subsection{Background Information}

Building 14 (B14) of the Praxair facility, located in Tonawanda, NY was used for uranium separation processes during the 1940's for the Manhattan Engineering District (MED), the predecessor to the Atomic Energy Commission (AEC). This site is currently under the cognizance of Bechtel National, Inc. (BNI), the Department of Energy (DOE) prime contractor for the Formerly Utilized Sites Remedial Action Program (FUSRAP). IDM Environmental Corp. was contracted by BNI to decontaminate the radioactive contamination (BNI1993) in Building 14 on surfaces and structures to the criteria established in DOE 5400.5 .

In prior site activities, contamination was detected within the low pressure air system associated with previous MED operations. This finding prompted an assessment of all process piping in B14 to support the building radiological release program. As part of this survey and decontamination program, process lines throughout B14 were evaluated and characterized for radioactive contamination. This report presents the results of the process piping survey and sampling investigation.

\subsection{Historical Information}

The Praxair facility was formerly owned and operated by Linde Air Products, formerly a subsidiary of Union Carbide Industrial Gases. From approximately 1943 to 1948 , MED contracted Linde Air Products to perform uranium separation operations because of its expertise in producing uranium salts used in the manufacture of various ceramic products. B14 was used primarily for laboratory and pilot plant studies during the operations period. After uranium separation operations ceased, most of the associated systems and components of the operation were removed. Documentation is not available to determine exact locations of uranium processes and the associated process piping. Due to the nature of the operations, temporary systems are believed to have been installed and subsequently removed. Piping originally designed and used for a specific service may have been later modified to accommodate other services, such as changing a low pressure air system into a vacuum system or cross-connecting systems based on needs.

Insulation, most of which is asbestos bearing, has been repaired, removed or replaced with various modifications over the life of the building. The original process piping systems have been modified to support the changing needs of the occupants. The building is now a modern laboratory with typical utilities and service piping systems.

\subsection{Scope of Project}

The scope of this project was to investigate process piping located within B14 of the Praxair facility. The radiological investigation included sampling and HpGe gamma spectroscopy analysis of process piping debris, and direct contamination measurements of external and internal piping components. Based on the review of the 


\section{B-6}

process piping system history, a site procedure (Attachment 1) was prepared to sample and survey representative process piping. Plan views of the Praxair site and Building 14 are shown as Attachment 2. Work performed for this investigation was performed in accordance with the IDM Site Specific Health \& Safety Plan (IDMSHP).

\subsection{Process Piping Radiological Investigation Approach}

\subsection{Survey Methodology}

The methodology to obtain representative survey measurements and samples of the process piping began by prioritizing piping into two areas of potential contamination; Group 1 categorized piping by assigning a high or low potential according to the radiological contamination conditions of the area in which they were installed, and Group 2, into high and low contamination potential based on historical information and the type of system (see Tables $2.1 \& 2.2$ ). Estimated quantities of each process piping system were generated for all areas in B14 and is presented as Attachment 6.

Table 2.1 Group 1 piping grouped by area contamination.

\begin{tabular}{|c||c|}
\hline Higher Contamination Areas & \multicolumn{1}{|c|}{ Lower Contamination Areas } \\
\hline Areas $9,12,13,14$ North and South & $\begin{array}{l}\text { Areas 2, 3, 4, 20A, 8, 10,11,15, 1st and 2nd } \\
\text { floor offices, large hallway }\end{array}$ \\
\hline
\end{tabular}

Table 2.2 Group 2 piping grouped by characteristics and contamination potential.

\begin{tabular}{|c|c|c|c|}
\hline \multicolumn{2}{|c|}{ Higher Potential for Contamination } & \multicolumn{2}{|c|}{ Lower Potential for Contamination } \\
\hline System & Comments & System & Comments \\
\hline $\begin{array}{l}\text { High } \\
\text { Pressure } \\
\text { Steam }\end{array}$ & $\begin{array}{l}\text { Heating process } \\
\text { systems, vacuum traps }\end{array}$ & Nitrogen Gas & Post MED, Pressurized \\
\hline $\begin{array}{l}\text { Low } \\
\text { Pressure } \\
\text { Steam }\end{array}$ & $\begin{array}{l}\text { Heating process } \\
\text { systems, vacuum traps }\end{array}$ & Natural Gas & Pressurized \\
\hline $\begin{array}{l}\text { Condensat } \\
\text { e Return }\end{array}$ & $\begin{array}{l}\text { Part of low and high } \\
\text { pressure steam lines }\end{array}$ & Oxygen & Post MED, Pressurized \\
\hline $\begin{array}{l}\text { Ventilation } \\
\text { Systems }\end{array}$ & $\begin{array}{l}\text { Overhead contamination } \\
\text { indicates prior } \\
\text { distribution of airborne } \\
\text { contamination }\end{array}$ & Potable Water & $\begin{array}{l}\text { Pressurized, washing } \\
\text { effect }\end{array}$ \\
\hline & & $\begin{array}{l}\text { Cooling Water } \\
\text { Supply \& } \\
\text { Return }\end{array}$ & $\begin{array}{l}\text { Pressurized, washing } \\
\text { effect }\end{array}$ \\
\hline & & $\begin{array}{l}\text { Hot Water } \\
\text { Supply \& } \\
\text { Retum } \\
\end{array}$ & $\begin{array}{l}\text { Pressurized, washing } \\
\text { effect }\end{array}$ \\
\hline . & & $\begin{array}{l}\text { Electrical } \\
\text { Conduit } \\
\end{array}$ & Accessible for survey \\
\hline
\end{tabular}




\section{B-7}

The general survey and sampling procedure was as follows:

- Biased surveys were performed in areas where potential leakage was evident, indicated by discoloration and scale deposits on insulation and external surfaces.

- Process piping system insulation (typically $A C M$ ) was removed to investigate the exterior piping under the insulation where exterior insulation was found to be contaminated. All ACM removals were performed in accordance with (12NYCRR56).

- Prior radiation survey data and knowledge of the historical use of the area provided a basis for the location of internal measurements.

- Because of the uncertainty of the installation and removal dates, all ventilation systems were accessed and surveyed both externally and internally for radioactive contamination. Internal surveys were conducted at various points within the ventilation systems for fixed and removable contamination.

- A large area internal smear or sample was performed at each system breech point and the sample analyzed by HpGe gamma spectroscopy to determine isotopic uranium contamination concentrations ( $\mathrm{pCi}$ or $\mathrm{pCi} / \mathrm{g}$ ).

3.0 Independent Verification Contractor (IVC)

Prior to the B14 process piping investigation, a meeting was held with the IVC (Lockheed Martin) management. The primary responsibility of the IVC is to provide QC verification and certification of B14 areas decontaminated and released by IDM for unrestricted use. The meeting discussed the plan for investigation of $B 14$ process piping systems. All concerns were identified and resoived prior to investigation activities. The following are the primary issues that were agreed upon and completed by IDM and the IVC.

3.1 IDM and IVC agreed to perform inter-comparisons of portable radiation detection instrumentation efficiencies and response between the two agencies. This comparison was completed with acceptable agreement.

3.2 IDM agreed to open and survey each of the four high potential concern process piping types at a minimum of six locations within the building and at a minimum of three locations for each of the low potential concern process piping.

4.0 Radiological Survey Findings and Results

The following is a summary of the radiological surveys performed in support of the B14 process piping investigation. Results of field measurements are presented in Attachment 3. Radiation detection instrumentation utilized by IDM in performance of field surveys is shown in Attachment 5 . 


\subsection{External Surfaces}

\section{B-8}

4.1.1 No removable contamination was detected on any of process piping.

4.1.2 Fixed contamination was detected on electrical conduit and steam piping. All surfaces were decontaminated to less than guideline values.

4.1.3 Asbestos insulation (ACM) was originally contaminated in the Corridor, Large Hall and Areas 2, and 3. The ACM insulation was either decontaminated or removed.

4.1.4 Electrical conduit, steam and natural gas piping in the north end Corridor was originally contaminated with levels of from $\approx 500$ to $1000 \mathrm{cpm}$ (32000 to $64000 \mathrm{dpm} / 100 \mathrm{~cm} 2$ ). All surfaces were decontaminated to within guideline values.

4.1.5 Non-insulated nitrogen pipe in the Large Hallway had levels of contamination up to $\approx 500 \mathrm{cpm}(32000 \mathrm{dpm} / 100 \mathrm{~cm} 2)$. All surfaces were decontaminated to within guideline values.

4.1.6 An external natural gas line in Area $14 \mathrm{SW}$ was originally contaminated with levels of $\approx 300$ to $1200 \mathrm{cpm}(19000$ to $77000 \mathrm{dpm} / 100 \mathrm{~cm} 2)$. Decontamination is scheduled for that line as of this time.

4.1.7 A remnant process pipe located in the overhead of Area 3 and 4 was originally contaminated up to $\approx 400 \mathrm{cpm}(26000 \mathrm{dpm} / 100 \mathrm{~cm} 2)$ has been decontaminated to within guideline values.

4.1.8 Contaminated ACM insulation on steam piping $(500 \mathrm{cpm})$ was removed in Area 2.

4.1.9 Electrical conduit lines within various areas of B14 contaminated up to $\approx 1200 \mathrm{cpm}(77000 \mathrm{dpm} / 100 \mathrm{~cm} 2)$ were decontaminated to within guideline values.

4.1.10 A water heater in Area 15 was found to be contaminated up to a maximum of $1000 \mathrm{cpm}(64000 \mathrm{dpm} / 100 \mathrm{~cm} 2)$. The unit was decontaminated to within guideline values.

\subsection{Internal Surfaces}

Approximately 145 samples of piping internals were anaiyzed by low level gamma spectroscopy. All process pipe internal samples anaiyzed by low level gamma spectroscopy were evaluated for total Uranium activity concentration in $\mathrm{pCi} / \mathrm{g}$ (for samples of residual dust or debris) or expressed as equivalent $\mathrm{dpm} / 100 \mathrm{~cm} 2$ for wipes of internal surfaces. The results of $\mathrm{HpGe}$ gamma spectroscopy analysis are presented in Attachment 4.

4.2.1 One internal debris/dust sample of a space heater intake plenum located in the overhead of Area 12 was determined at $88 \mathrm{pCi} / \mathrm{g}$; the remaining surface 
Process Piping Radiological Investigation, Praxair Building 14, Tonawanda, NY

debris and dust was removed from the system.

4.2.2 The Corridor ventilation space heater was contaminated to a maximum of $500,000 \mathrm{dpm} / 100 \mathrm{~cm} 2$. The space heater was removed and packaged for disposal.

5.0 Summary

During calendar year 1997, the process piping and ventilation systems within Praxair B14 were radiologically characterized by performance of surveys, samples and historical records review. Process piping and ventilation systems identified as being radiologically contaminated were then decontaminated or removed and packaged for disposal.

Final survey data demonstrate with a high degree of confidence (at the $95 \%$ confidence level) that no significant radioactivity attributable to the MED uranium processing operations remains on or within the investigated process pipe or ventilation systems of B14.

The final radiological status of the B14 process pipe and ventilation systems are such that overall residual activity from uranium operations is significantly less than the guideline values, does not pose a health and safety hazard to operating personnel, and the process piping and ventilation systems meet all requirements for release for unconditional use.

\subsection{References}

(IDM97) IDM Process Piping Investigation Procedure, dated 4/16/97.

(IDMSHP) IDM Site Specific Health \& Safety Plan, Rev 1.7, dated 9/4/97.

(12NYCRR56) Asbestos Removal NY Code Rule 56

(BNI1993) Remedial Investigation (RI) Report. 
B-10

B14 Process Piping Investigation

\section{Attachment 1}

Process Piping Investigation Procedure 
B-11

Building 14 / Praxair

Process Piping Radiological Investigation

$\begin{array}{ll}\text { Date } & \text { April 16, } 1997 \\ \text { Task Order \# } & : 129-S C-5.63-023 \\ \text { Prepared } & : \text { Mark Cafouras / Peter Biesiadecki }\end{array}$

Purpose: IDM Radiological Investigation and Clearance of Building 14 Process Piping with the Independent Verification Contractor (IVC) concurrence to release from any future radiological concerns

References: Prudent Practices for Handling Hazardous Chemicals in Laboratories IDM Procedure - Line Purge and Line Break Safety Procedure IDM Lockout / Tagout Procedure Applicable MSDS sheets for the appropriate process line gases Praxair Hazardous Work Permit / Notification IDM asbestos removal procedure Code Rule 56: Requirements for Asbestos Sampling \& Removal

Preliminary: IDM Management Safety consultation and review

Praxair approval for line valve isolations with field verification for lockout/tagout IDM Site Safety review of IDM Task Hazard Analysis (THA) with site personnel Radiation Work Permit preparation and safety review with workforce Pre-job Briefing

\section{Work Plan / Procedure for Process Piping Investigation}

\subsection{Introduction}

The purpose of this procedure is to purge, survey, and sample the process piping in Bld 14 for radiological contamination both externally and internally for release to the Independent Verification Contractor from any radiological concerns.

2.0 BNI / Praxair Site Safety have been briefed and concurred on intended operations to isolate and survey the process piping. In each new area, IDM will communicate and brief BNF / Praxair on intended operations to coordinate safety / work operations with the Building 14 tenants. IDM will post the area to limit site personnel access during process survey operations. Praxair will generate and post their own Hazardous Work Permit to inform their personnel of intended operations.

3.0 Praxair will identify and sign-off the appropriate process system isolation valves to allow IDM to isolate the affected process system. IDM will maintain Positive control with Praxair concurrence by tagging the appropriate valves shut and the removal of the affected system operating hand-wheels during the process piping investigation.

4.0 IDM will generate a Task Hazard Analysis and Radiation Work Permit to identify various 
B-12

process piping hazards including chemical and radiological concerns. A review with appropriate work personnel will be communicated with signoff at the pre-job briefing prior to the commencement of work. The affected area will be posted to limit access to appropriate personnel. Scaffolding and/or the Genie Lift will be built, inspected, and used to access the appropriate breech / vent points.

5.0 Process Piping is divided into 2 categories -High Potential and Low Potential from a radiological concern.

High Potential Lines include: 1. Low pressure air

2. High pressure steam

3. Low pressure steam

4. Steam Condensate

Low Potential Lines include: 5. Natural gas

6. Oxygen

7. Hot \& Cold water

8. Nitrogen gas-various pressures

Additionally, Bld 14 'Areas' are divided into "High Profile" areas which include: "Areas" 12,13,14N, 14S, and Area 9. with others identified "Low Profile" areas unless otherwise identified.

Depending on accessibility, IDM's goal is 6 internal access survey points for Hi-potential process lines and 3 access points for Low-potential process lines in Building 14.

Externally, all insulated process piping will be radiologically surveyed. When applicable, a certain percentage of insulated asbestos process piping insulation will be removed and externally surveyed at probable unions, drains, strainers to verify process system leaks and insure radiologically no contamination exists under the insulation following all applicable asbestos regulations. Furthermore, HVAC systems will be tagged, inspected and surveyed as required.

General Safety Precautions will include but not limited to the following for each area entered:

- After the Work Area Foreman has identified, tagged, and traced the appropriate process line to be breached \& investigated, he is the only individual authorized to direct which valves / unions are to be opened and closed during the evolution.

- All personnel involved will know where appropriate emergency equipment (i.e. fire extinguishers), alarms, and appropriate emergency phone \# etc. are located within each new work area entered.

- No smoking, open flames, sources of heat, or spark producing equipment will be allowed or operated during the following process line venting or re-assembly, notably natural gas and oxygen. Grounding straps will be used during venting operations to eliminate static- produced sparks. Personnel should ground tools to adjacent nonflammable metal piping prior to starting hazardous work. Soapy water \& explosimeters are the preferred method for leak checking various unions / valves 
during re-assembly.

- No oil or grease will be used on any process piping (i.e. oxygen) or oxygen tanks. This will include the use of new work gloves during disassembly and re-assembly by the workforce. Care will be exercised never to interchange oxygen valves, regulators, etc with any other intended use.

- Oxygen and Natural gas process lines will never be vented sequentially but preferably on different days.

- Extreme caution will be exercised when breaching steam systems due to burns and the prevalence of isolation valve leak-bye.

- The Radiation Work Permit will identify dressout requirements which may be changed by Site Safety based on the various chemical safety considerations of the process systems.

\subsection{PROCESS PIPING PROCEDURE:}

6.0.1 The internal radiological survey of Process piping in Bld 14 will require isolation and evacuation for access. Process piping with hazardous constituents (i.e. natural gas, oxygen, \& nitrogen) will require venting and purging with inert nitrogen gas (or low pressure air as applicable for nitrogen) to an elevated area outside the exterior of the building (away from any personnel, air intakes into the bld, or sources of ignition) prior to breaching. After the Praxair authorized person has authorized and field verified the process line isolation valves to be shut, IDM will hang the approved tags on the process lines to be isolated and verify tagged shut isolation valves with the removal of the operating handle to maintain positive control.

6.0.2 IDM will start with non-hazardous process lines (hot/cold water \& low pressure air) followed by steam and condensate as applicable to familiarize the work crews with the operations and safety procedures.

6.0.3 IDM will verify initial hazardous (natural gas, oxygen, \& nitrogen) process piping conditions at the unions / valves with the explosimeter to ensure a non-hazardous conditions exists prior to start of work. A pressure test will be performed on the appropriate system via the system piping down-comers and a 2 stage gauged nitrogen gas cylinder (mounted and chained in a mobile cart) to test the inregnty of the tagged isolation valves / system for leakage.

6.0.4 If successful, the appropriate process piping lines will be isolated and purged to remove their hazardous characteristics. All special safety precautions for Natural Gas, Oxygen, and Nitrogen will be followed during the procedure. Areas will be posted to eliminate flammability hazards, smoking, sparks, oxygen deficiency during all gas venting operations.

6.0.5 The systems (natural gas, oxygen, nitrogen) will be evacuated first slowly by natural venting, followed with purging (@5-10 psig > system pressure with nitrogen or compressed air as applicable) approximately 2 calculated volumes of gas to the outside atmosphere via a rubber air hose connected to an available system down-comer. The rubber vent hose will be grounded to earth ground. Two 
B-14

combination LEL / Oxygen meters will be used for this purging- one in the area where personnel are working inside the building and the other monitoring the vented gas to the outside to verify when the process piping is fully evacuated and vented. Other low points and down-comers will also be required to be sequentially vented, to ensure removal of all hazardous constituents in the same manner. Other process piping lines (i.e. cold \& hot water, steam) will be isolated, vented, and drained to Bld-14 drains prior to breech.

6.0.4 After the affected process line has been completed evacuated and verified by IDM site safety, the process line will be breached at available applicable access points including unions, strainers, and pipe caps with IDM site safety continuously monitoring for any hazardous conditions. RadCon will perform the internal survey / sampling of the internal breached areas. After completion of the radiological survey, the system will be reassembled and closed in preparation for returning to service.

6.0.5 A final pressure test will be performed before opening applicable isolation valves with bottled gas to verify system integrity and tested with soapy water at the breached sampling points. The oxygen lines will be purged and vented with bottled oxygen while the low pressure air will be tested and replaced with compressed air. The natural gas lines will not need to be purged. If successful and after authorization to remove the tagged isolation valves, the work area foreman will return the system to service.

Prepared By:

\author{
Prepared By:
}

Mark Cafouras

Ion Technology, Inc

Peter Biesiadecki

IDM Environmental, Inc

Reviewed / Approved By:

Date: $5 / 19 / 97$

IDM Site Superintendent

Date: $5 / 19 / 97$

Joe Dinardo

Date 5/19/97

IDM H \& S Representative 


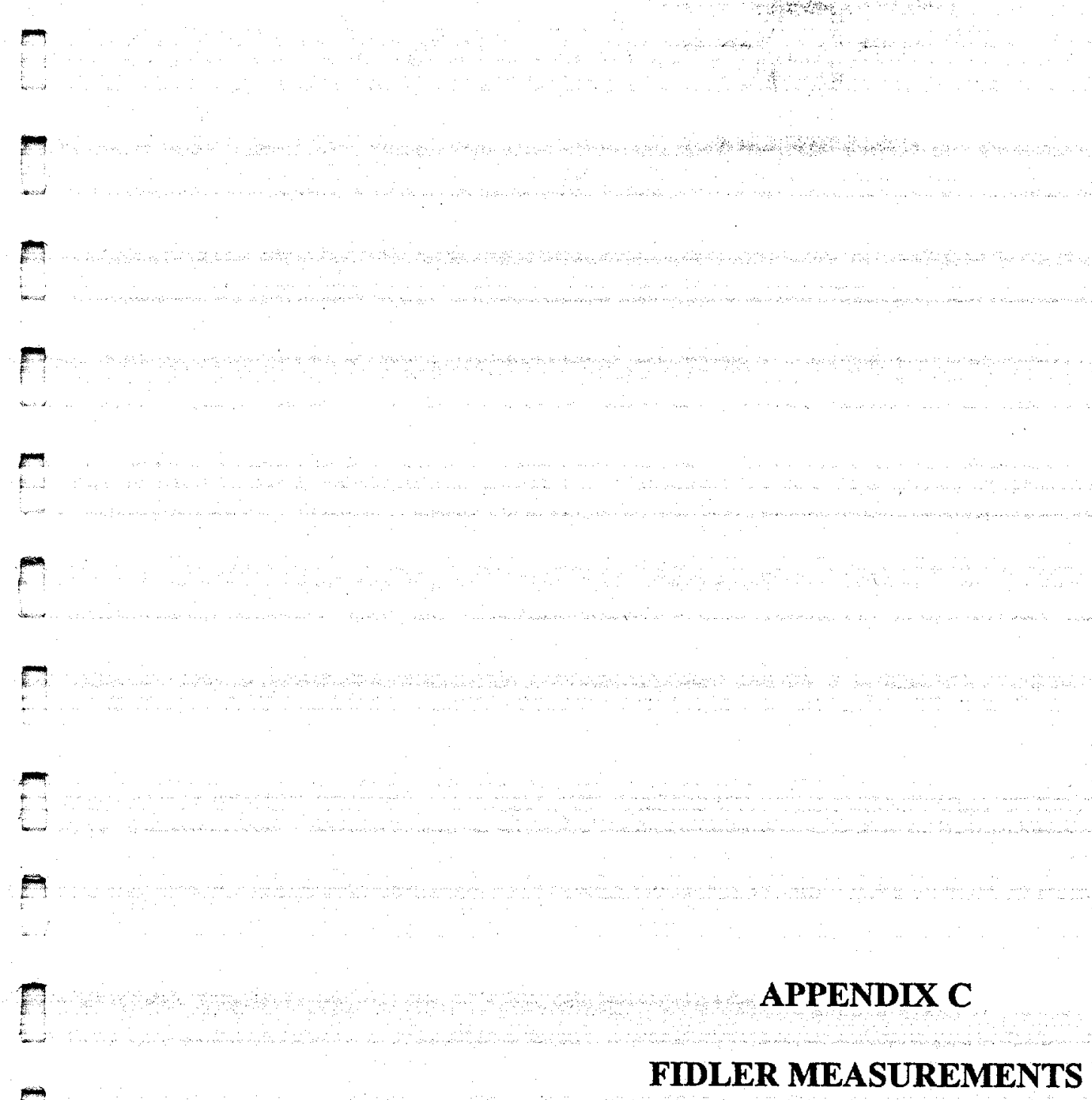




\section{C-3}

Table C.1. FIDLER measurements with Detector A003Y at Building 14 (Data not normalized ${ }^{a}$ )

\begin{tabular}{|c|c|c|c|c|c|}
\hline Area & North $^{b}$ & East $^{b}$ & $\begin{array}{l}\text { Counts } \\
(1 \mathrm{~min}) \\
\end{array}$ & Date & Detector \\
\hline 8 & 0 & 5 & 12,352 & $4-15-97$ & $\mathrm{~A} 003 \mathrm{Y}$ \\
\hline 8 & 2 & 5 & 11,941 & $4-15-97$ & $\mathrm{~A} 003 \mathrm{Y}$ \\
\hline 8 & 4 & 5 & 12,561 & $4-15-97$ & $\mathrm{~A} 003 \mathrm{Y}$ \\
\hline 8 & 6 & 5 & 12,720 & $4-15-97$ & $\mathrm{~A} 003 \mathrm{Y}$ \\
\hline 8 & 8 & 5 & 11,450 & $4-15-97$ & $A 003 Y$ \\
\hline 8 & 10 & 5 & 11,988 & $4-15-97$ & $\mathrm{~A} 003 \mathrm{Y}$ \\
\hline 8 & 12 & 5 & 8,900 & $4-15-97$ & $\mathrm{~A} 003 \mathrm{Y}$ \\
\hline 8 & 14 & 5 & 9,158 & $4-15-97$ & $\mathrm{~A} 003 \mathrm{Y}$ \\
\hline 8 & 4 & 1 & 12,308 & $4-15-97$ & $A 003 Y$ \\
\hline 8 & 6 & 1 & 13,051 & $4-15-97$ & $A 003 Y$ \\
\hline 8 & 8 & 1 & 12,245 & $4-15-97$ & $\mathrm{~A} 003 \mathrm{Y}$ \\
\hline 8 & 10 & 1 & 12,353 & $4-15-97$ & $A 003 Y$ \\
\hline 8 & 12 & 1 & 11,047 & $4-15-97$ & $\mathrm{~A} 003 \mathrm{Y}$ \\
\hline 10 & 14 & 12 & 11,178 & $4-15-97$ & $A 003 Y$ \\
\hline 10 & 0 & 10.5 & 11,517 & $4-15-97$ & $\mathrm{~A} 003 \mathrm{Y}$ \\
\hline 10 & 2 & 10.5 & 11,268 & $4-15-97$ & $\mathrm{~A} 003 \mathrm{Y}$ \\
\hline 10 & 4 & 10.5 & 12,062 & $4-15-97$ & $\mathrm{~A} 003 \mathrm{Y}$ \\
\hline 10 & 6 & 10.5 & 11,990 & $4-15-97$ & $\mathrm{~A} 003 \mathrm{Y}$ \\
\hline 10 & 8 & 10.5 & 11,086 & $4-15-97$ & $A 003 Y$ \\
\hline 10 & 10 & 10.5 & 10,317 & $4-15-97$ & $\mathrm{~A} 003 \mathrm{Y}$ \\
\hline 10 & 1 & 1 & 8,527 & $4-15-97$ & $\mathrm{~A} 003 \mathrm{Y}$ \\
\hline 10 & 1 & 3 & 9,600 & $4-15-97$ & $\mathrm{~A} 003 \mathrm{Y}$ \\
\hline 10 & 1 & 5 & 9,787 & $4-15-97$ & $A 003 Y$ \\
\hline 10 & 1 & 7 & 8,875 & $4-15-97$ & $\mathrm{~A} 003 \mathrm{Y}$ \\
\hline 11 & 5 & 7 & 10,127 & $4-15-97$ & $\mathrm{~A} 003 \mathrm{Y}$ \\
\hline 11 & 5 & 5 & 10,719 & $4-15-97$ & $\mathrm{~A} 003 \mathrm{Y}$ \\
\hline 11 & 5 & 3 & 10,164 & $4-15-97$ & $A 003 Y$ \\
\hline 11 & 5 & 1 & 9,731 & $4-15-97$ & $\mathrm{~A} 003 \mathrm{Y}$ \\
\hline
\end{tabular}




\section{C-4}

Table C.1 (continued)

(Data not normalized ${ }^{\text {) }}$

\begin{tabular}{|c|c|c|c|c|c|}
\hline Area & North $^{b}$ & East $^{b}$ & $\begin{array}{r}\text { Counts } \\
(1 \mathrm{~min}) \\
\end{array}$ & Date & Detector \\
\hline $20 \mathrm{~B} / 20 \mathrm{~B}-1$ & 0 & 9 & 9,203 & $10-28-96$ & $\mathrm{~A} 003 \mathrm{Y}$ \\
\hline $20 \mathrm{~B} / 20 \mathrm{~B}-1$ & 0 & 8 & 8,807 & $10-28-96$ & $\mathrm{~A} 003 \mathrm{Y}$ \\
\hline $20 \mathrm{~B} / 20 \mathrm{~B}-1$ & 0 & 7 & 8,594 & $10-28-96$ & $\mathrm{~A} 003 \mathrm{Y}$ \\
\hline $20 \mathrm{~B} / 20 \mathrm{~B}-1$ & 0 & 6 & 6,801 & $10-28-96$ & $\mathrm{~A} 003 \mathrm{Y}$ \\
\hline $20 \mathrm{~B} / 20 \mathrm{~B}-1$ & 0 & 5 & 10,331 & $10-28-96$ & $\mathrm{~A} 003 \mathrm{Y}$ \\
\hline $20 \mathrm{~B} / 20 \mathrm{~B}-1$ & 0 & 4 & 9,862 & $10-28-96$ & $\mathrm{~A} 003 \mathrm{Y}$ \\
\hline $20 \mathrm{~B} / 20 \mathrm{~B}-1$ & 0 & 3 & 7,486 & $10-28-96$ & $\mathrm{~A} 003 \mathrm{Y}$ \\
\hline $20 \mathrm{~B} / 20 \mathrm{~B}-1$ & 0 & 2 & 9,916 & $10-28-96$ & $\mathrm{~A} 003 \mathrm{Y}$ \\
\hline $20 \mathrm{~B} / 20 \mathrm{~B}-1$ & 0 & 1 & 8,407 & $10-28-96$ & $\mathrm{~A} 003 \mathrm{Y}$ \\
\hline $20 \mathrm{~B} / 20 \mathrm{~B}-1$ & 0 & 0 & 8,396 & $10-28-96$ & $\mathrm{~A} 003 \mathrm{Y}$ \\
\hline $20 \mathrm{~B} / 20 \mathrm{~B}-1$ & 3 & 9 & 8,296 & $10-28-96$ & $\mathrm{~A} 003 \mathrm{Y}$ \\
\hline $20 \mathrm{~B} / 20 \mathrm{~B}-1$ & 3 & 8 & 6,974 & $10-28-96$ & $\mathrm{~A} 003 \mathrm{Y}$ \\
\hline $20 \mathrm{~B} / 20 \mathrm{~B}-1$ & 3 & 7 & 8,499 & $10-28-96$ & $\mathrm{~A} 003 \mathrm{Y}$ \\
\hline $20 \mathrm{~B} / 20 \mathrm{~B}-1$ & 3 & 6 & 9,180 & $10-28-96$ & $\mathrm{~A} 003 \mathrm{Y}$ \\
\hline $20 \mathrm{~B} / 20 \mathrm{~B}-1$ & 3 & 5 & 8,821 & $10-28-96$ & $\mathrm{~A} 003 \mathrm{Y}$ \\
\hline $20 \mathrm{~B} / 20 \mathrm{~B}-1$ & 3 & 4 & 8,801 & $10-28-96$ & $A 003 Y$ \\
\hline $20 \mathrm{~B} / 20 \mathrm{~B}-1$ & 3 & 3 & 6,806 & $10-28-96$ & $\mathrm{~A} 003 \mathrm{Y}$ \\
\hline $20 \mathrm{~B} / 20 \mathrm{~B}-1$ & 3 & 2 & 9,253 & $10-28-96$ & $\mathrm{~A} 003 \mathrm{Y}$ \\
\hline 20B/20B-1 & 3 & 1 & 9,489 & $10-28-96$ & $\mathrm{~A} 003 \mathrm{Y}$ \\
\hline $20 \mathrm{~B} / 20 \mathrm{~B}-1$ & 3 & 0 & 9,212 & $10-28-96$ & $\mathrm{~A} 003 \mathrm{Y}$ \\
\hline $20 \mathrm{~B} / 20 \mathrm{~B}-1$ & 4 & 0 & 8,590 & $10-28-96$ & $\mathrm{~A} 003 \mathrm{Y}$ \\
\hline 20B/20B-1 & 4 & 1 & 9,284 & $10-28-96$ & $\mathrm{~A} 003 \mathrm{Y}$ \\
\hline $20 \mathrm{~B} / 20 \mathrm{~B}-1$ & 4 & 2 & 8,779 & $10-28-96$ & $\mathrm{~A} 003 \mathrm{Y}$ \\
\hline $20 \mathrm{~B} / 20 \mathrm{~B}-1$ & 4 & 3 & 8,183 & $10-28-96$ & $\mathrm{~A} 003 \mathrm{Y}$ \\
\hline $20 \mathrm{~B} / 20 \mathrm{~B}-1$ & 4 & 4 & 8,477 & $10-28-96$ & A003Y \\
\hline
\end{tabular}

${ }^{a}$ Measurements from Detector A003Y can only be compared with other measurements from the same detector.

${ }^{b}$ Meters north and east of southwest corner (NO, EO). 


\section{C-5}

Table C.2. FIDLER measurements with Detector A005Y at Building 14 (Data not normalized ${ }^{a}$ )

\begin{tabular}{|c|c|c|c|c|c|}
\hline Area & North $^{b}$ & East $^{b}$ & $\begin{array}{l}\text { Counts } \\
\text { (1 min) }\end{array}$ & Date & Detector \\
\hline 8 & 0 & 7 & 15,094 & $4-15-97$ & $\mathrm{~A} 005 \mathrm{Y}$ \\
\hline 8 & 2 & 7 & 13,837 & $4-15-97$ & $\mathrm{~A} 005 \mathrm{Y}$ \\
\hline 8 & 4 & 7 & 15,338 & $4-15-97$ & A005Y \\
\hline 8 & 6 & 7 & 14,472 & $4-15-97$ & $\mathrm{~A} 005 \mathrm{Y}$ \\
\hline 8 & 8 & 7 & 13,241 & $4-15-97$ & $\mathrm{~A} 005 \mathrm{Y}$ \\
\hline 8 & 10 & 7 & 12,877 & $4-15-97$ & A005Y \\
\hline 8 & 2 & 3 & 11,592 & $4-15-97$ & $\mathrm{~A} 005 \mathrm{Y}$ \\
\hline 8 & 4 & 3 & 14,447 & $4-15-97$ & $\mathrm{~A} 005 \mathrm{Y}$ \\
\hline 8 & 6 & 3 & 13,681 & $4-15-97$ & A005Y \\
\hline 8 & 8 & 3 & 11,130 & $4-15-97$ & A005Y \\
\hline 8 & 10 & 3 & 12,741 & $4-15-97$ & $\mathrm{~A} 005 \mathrm{Y}$ \\
\hline 8 & 12 & 3 & 11,464 & $4-15-97$ & $\mathrm{~A} 005 \mathrm{Y}$ \\
\hline 8 & 14 & 3 & 10,668 & $4-15-97$ & $A 005 Y$ \\
\hline 10 & 0 & 12 & 12,460 & $4-15-97$ & $\mathrm{~A} 005 \mathrm{Y}$ \\
\hline 10 & 2 & 12 & 12,721 & $4-15-97$ & $\mathrm{~A} 005 \mathrm{Y}$ \\
\hline 10 & 4 & 12 & 13,681 & $4-15-97$ & $\mathrm{~A} 005 \mathrm{Y}$ \\
\hline 10 & 6 & 12 & 13,342 & $4-15-97$ & $A 005 Y$ \\
\hline 10 & 8 & 12 & 12,062 & $4-15-97$ & $\mathrm{~A} 005 \mathrm{Y}$ \\
\hline 10 & 10 & 12 & 13,266 & $4-15-97$ & $\mathrm{~A} 005 \mathrm{Y}$ \\
\hline 10 & 2 & 8 & 12,704 & $4-15-97$ & $\mathrm{~A} 005 \mathrm{Y}$ \\
\hline 10 & 4 & 8 & 13,581 & $4-15-97$ & $\mathrm{~A} 005 \mathrm{Y}$ \\
\hline 10 & 6 & 8 & 13,841 & $4-15-97$ & $\mathrm{~A} 005 \mathrm{Y}$ \\
\hline 10 & 8 & 8 & 13,457 & $4-15-97$ & $A 005 Y$ \\
\hline 10 & 10 & 8 & 13,121 & $4-15-97$ & $\mathrm{~A} 005 \mathrm{Y}$ \\
\hline 11 & 3 & 8 & 9,536 & $4-15-97$ & $A 005 Y$ \\
\hline 11 & 5 & 8 & 11,008 & $4-15-97$ & $\mathrm{~A} 005 \mathrm{Y}$ \\
\hline 11 & 7 & 8 & 10,685 & $4-15-97$ & A005Y \\
\hline 11 & 9 & 8 & 10,145 & $4-15-97$ & $\mathrm{~A} 005 \mathrm{Y}$ \\
\hline
\end{tabular}




\section{C-6}

Table C.2 (continued)

(Data not normalized ${ }^{a}$ )

\begin{tabular}{|c|c|c|c|c|c|}
\hline Area & North ${ }^{b}$ & East $^{b}$ & $\begin{array}{l}\text { Counts } \\
(1 \mathrm{~min})\end{array}$ & Date & Detector \\
\hline $20 \mathrm{~B} / 20 \mathrm{~B}-1$ & 1 & 9 & $10,451^{c}$ & $10-28-96$ & A005Y \\
\hline $20 \mathrm{~B} / 20 \mathrm{~B}-1$ & 1 & 8 & 8,051 & $10-28-96$ & $\mathrm{~A} 005 \mathrm{Y}$ \\
\hline $20 \mathrm{~B} / 20 \mathrm{~B}-1$ & 1 & 7 & 9,807 & $10-28-96$ & $\mathrm{~A} 005 \mathrm{Y}$ \\
\hline $20 \mathrm{~B} / 20 \mathrm{~B}-1$ & 1 & 6 & 7,486 & $10-28-96$ & $\mathrm{~A} 005 \mathrm{Y}$ \\
\hline $20 \mathrm{~B} / 20 \mathrm{~B}-1$ & 1 & 5 & 9,877 & $10-28-96$ & $\mathrm{~A} 005 \mathrm{Y}$ \\
\hline $20 \mathrm{~B} / 20 \mathrm{~B}-1$ & 1 & 4 & $10,112^{c}$ & $10-28-96$ & $\mathrm{~A} 005 \mathrm{Y}$ \\
\hline $20 \mathrm{~B} / 20 \mathrm{~B}-1$ & 1 & 3 & 7,070 & $10-28-96$ & $\mathrm{~A} 005 \mathrm{Y}$ \\
\hline $20 \mathrm{~B} / 20 \mathrm{~B}-1$ & 1 & 2 & 10,772 & $10-28-96$ & A005Y \\
\hline $20 \mathrm{~B} / 20 \mathrm{~B}-1$ & 1 & 1 & 10,909 & $10-28-96$ & A005Y \\
\hline $20 \mathrm{~B} / 20 \mathrm{~B}-1$ & 1 & 0 & 10,955 & $10-28-96$ & $\mathrm{~A} 005 \mathrm{Y}$ \\
\hline $20 \mathrm{~B} / 20 \mathrm{~B}-1$ & 2 & 0 & $10,508^{c}$ & $10-28-96$ & $\mathrm{~A} 005 \mathrm{Y}$ \\
\hline $20 \mathrm{~B} / 20 \mathrm{~B}-1$ & 2 & 1 & 10,386 & $10-28-96$ & AOOSY \\
\hline $20 \mathrm{~B} / 20 \mathrm{~B}-1$ & 2 & 2 & $10,411^{c}$ & $10-28-96$ & $\mathrm{~A} 005 \mathrm{Y}$ \\
\hline 20B/20B-1 & 2 & 3 & 7,139 & $10-28-96$ & $\mathrm{~A} 005 \mathrm{Y}$ \\
\hline $20 \mathrm{~B} / 20 \mathrm{~B}-1$ & 2 & 4 & 9,797 & $10-28-96$ & $\mathrm{~A} 005 \mathrm{Y}$ \\
\hline $20 \mathrm{~B} / 20 \mathrm{~B}-1$ & 2 & 5 & 9,456 & $10-28-96$ & $\mathrm{~A} 005 \mathrm{Y}$ \\
\hline $20 \mathrm{~B} / 20 \mathrm{~B}-1$ & 2 & 6 & 6,964 & $10-28-96$ & $\mathrm{~A} 005 \mathrm{Y}$ \\
\hline $20 \mathrm{~B} / 20 \mathrm{~B}-1$ & 2 & 7 & 8,676 & $10-28-96$ & $\mathrm{~A} 005 \mathrm{Y}$ \\
\hline $20 \mathrm{~B} / 20 \mathrm{~B}-1$ & 2 & 8 & 8,739 & $10-28-96$ & $\mathrm{~A} 005 \mathrm{Y}$ \\
\hline $20 \mathrm{~B} / 20 \mathrm{~B}-1$ & 2 & 9 & $7,726^{c}$ & $10-28-96$ & $\mathrm{~A} 005 \mathrm{Y}$ \\
\hline $20 \mathrm{~B} / 20 \mathrm{~B}-1$ & 4 & 9 & $8,911^{d}$ & $10-28-96$ & $\mathrm{~A} 005 \mathrm{Y}$ \\
\hline $20 \mathrm{~B} / 20 \mathrm{~B}-1$ & 4 & 8 & 9,363 & $10-28-96$ & $\mathrm{~A} 005 \mathrm{Y}$ \\
\hline $20 \mathrm{~B} / 20 \mathrm{~B}-1$ & 4 & 7 & 9,810 & $10-28-96$ & $\mathrm{~A} 005 \mathrm{Y}$ \\
\hline $20 \mathrm{~B} / 20 \mathrm{~B}-1$ & 4 & 6 & 8,026 & $10-28-96$ & $\mathrm{~A} 005 \mathrm{Y}$ \\
\hline $20 \mathrm{~B} / 20 \mathrm{~B}-1$ & 4 & 5 & 9,588 & $10-28-96$ & $\mathrm{~A} 005 \mathrm{Y}$ \\
\hline
\end{tabular}

${ }^{a}$ Measurements from Detector A005Y can only be compared with other measurements from the same detector.

Meters north and east of southwest corner (NOEO).

Near wall.

${ }^{d}$ Comer 


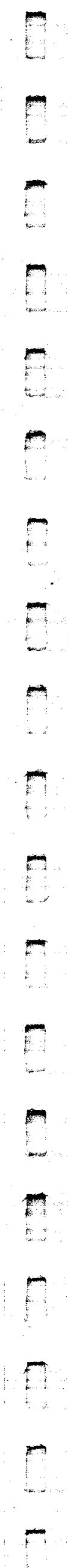

APPENDIX D

CORRESPONDENCE 
OAK RIDGE NATIONAL LABORATORY

MANAGED BY LOCKHEED MARTIN ENERGY AESEARCH CORPORATION

FOR THE US. DEPARTMENT OF ENERGY

POST OFFTCE 80X 2000

ONK RADGE TN 37831

December 30, 1996

Dr. W. Alexander Williams

Designation and Certification Manager

Office of Eastem Area Programs

Cloverieaf Building, (EM-421)

Department of Energy

19901 Germantown Road

Germantown. Maryland 20874-1290

Dear Dr. Williams:

\section{Contract DE-AC05.960R22464, Verification Surveys Conducted at Former Linde Site}

In October 1996 personnel from the Oak Ridge National Laboratory conducted verification surveys at the former Linde site in Tonawanda. New York. A request was made for Oak Ridge National Laboratory to verify several areas in Building 14. All areas surveyed met Department of Energy cleanup criterion.

We have released Areas $20 \mathrm{~A}$ East, 20B, 20B-1, and $20 \mathrm{C}$ as being below Department of Energy surface guidelines (see map). Additionaily, areas in 20A East, where the concrete floor was removed, met approved Department of Energy guidelines for uranium soil contamination in the state of New York. A total of seven sampies were taken from this area. However, we have not verified any other subsurfaces at the former Linde site.

Overall this particular project went quite well. The remediation contractor and technical support personnel worked together and did a good job of characterizing the areas and removing the contamination before the Oak Ridge National Laboratory verification team arrived.

If you have any questions please contact me at (423) $576-4108$.

Sincereiy,

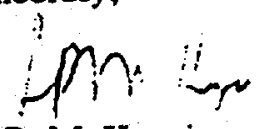

S. P. McKenzie

Measurement Applicarions and Development Group

SPM:lec

Enciosure(s): Former Linde Site Building 14

c. R. D. Foley

M. E. Murray

L. L. Baldy (BNI)

Rc-File 
D-4

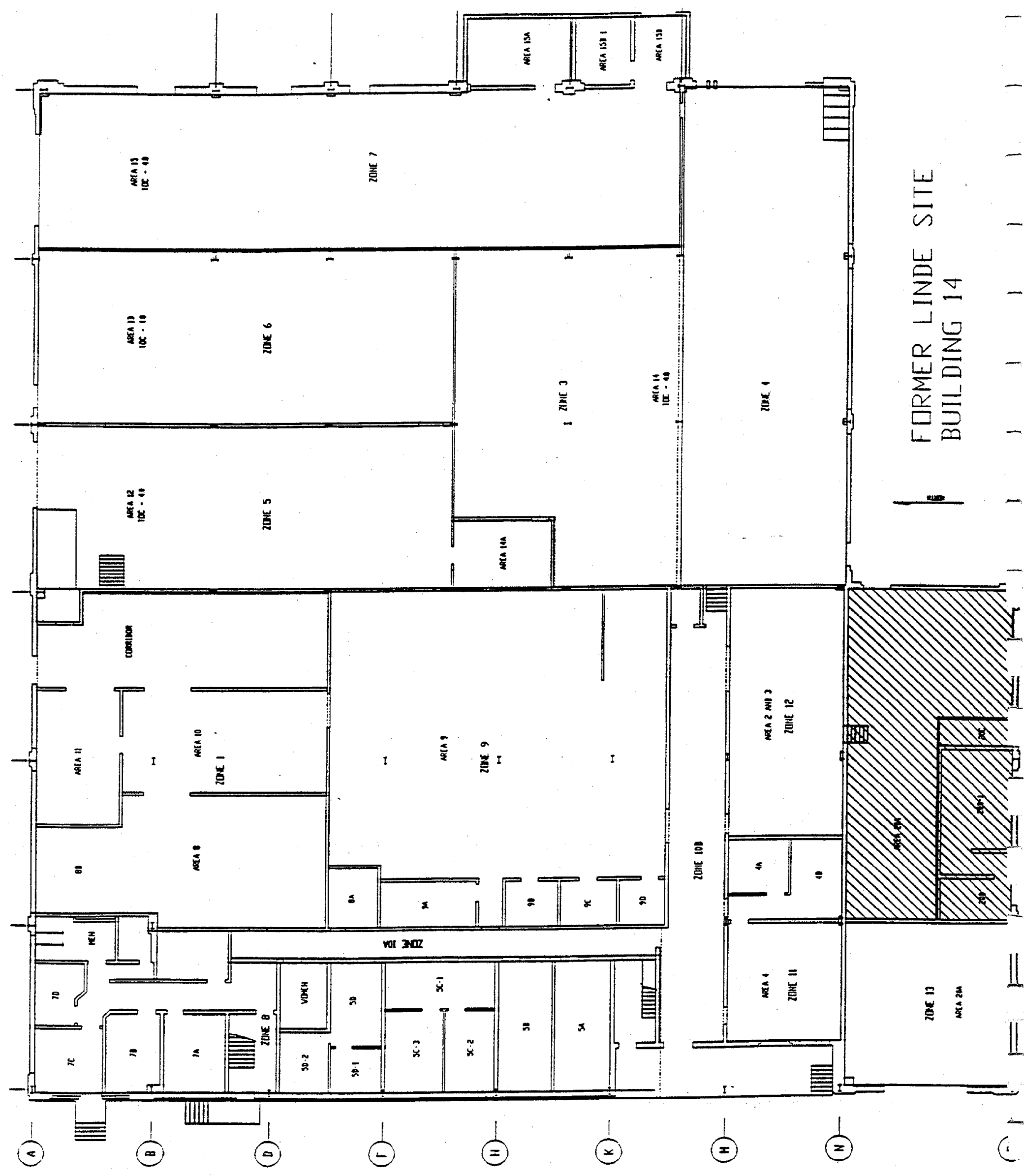




\section{most comes cox 200}

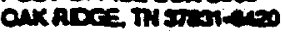

May 5, 1997

Dr. W. Alexander Williams

Designation and Certification Manager

Office of Eastem Area Programs

Cloverieaf Building, EM-421

Deparument of Energy

19901 Germantown Road

Germantown, Maryland 20874-1290

Dear Dr. Williams:

Contract DE-A C05-960R22464, Verification of Building 14, Praxair Site

As a result of meeting with the Bechtel National, Incorporated, New York team, and IDM Environmental Corporation (the Turnikey Subcontractor for Building 14), we have chosen this form of communicating the results of our verification surveys in this building. This assists both Bechtel and IDM Environmental Corporation in their planning. Therefore, you will be receiving a series of letters clearing the areas that pass our Independent Verification surveys. During the week of April 14, an Independent Verification Contractor survey was conducted in certain areas in this building. The resuits are as follows:

1. We have deciared the second floor as being below Department of Energy guidelines.

2. All above ground surfaces in areas 8, 10, and 11, are also released. No subsurface investigations have been conducted.

3. All surfaces above -1 foot of the wall/floor interface in area 12 were also released as below guidelines, with the exception of one "hot spot" on the lower horkontal surface, on the uppermost horizontal I-beam. Both Bechtel and IDM Environmental Corporation was made aware of this area and pending clean-up and survey of this area by Safety and Ecology Corporation, we would be willing to reiease this area. According to IDM Environmental Corporation the ledge that runs along the west wall and will be demolished with the floor (several areas above guidelines were found during a preliminary scan of the ledge).

4. Finally, in area 13, all surfaces above -1 foot of the floor/wall interface were dectared below guidelines with the exception of an I-beam that runs along the west wall. Hot spots were found along the beam on the lower horizontal surface closest to the wall. This area will need further decontamination.

Please call S. P. McKenzie (423) 576-4108, if additional information is needed.

Sincerely,

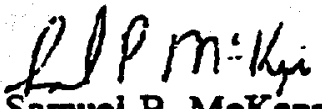

Samuel P. MeKenzie

Measurement Applications and Development Group

SPM

c: $\quad$ S. K Amrit, BNI

R. D. Foley

File. Re 
July 30,1997

Dr. W. Alexander Williams

Designation and Certification Manager

Office of Eastern Area Programs

Cloverieaf Building, (EM-421)

Department of Energy

19901 Germantown Road

Germantown, Maryiand 20874-1290

Dear Dr. Williams:

\section{Status of Cleanup of Former Linde Site in Tonawanda, New York}

This is the second letter in a series of updates concerning the status of the cleanup of the former Linde Site in Tonawanda, New York. We have chosen this method of notification to expedite the remediation process. Although the areas in building 14 were not initially prepared for our survey, we were able to complete our activities during this survey trip. Full reports will be prepared for each building after they are completed.

During the week of July 14, 1997, an Independent Verification Contractor (IVC) was conducted on certain areas in building 14 , and the exterior of building 31 . In addition, a verification survey was conducted on an above-ground tank near the former site of building 38 . The results are as follows:

- We have verified the above-ground tank (metal surfaces oniy) as being below Department of Energy surface guidelines.

- The exterior of building 31 (exterior walls and roof) was also verified as not exceeding Department of Energy guideiines, with the exception of the area behind the transformer, as identified by Bechtel's radiological support subcontractor. The entire building is now verinied, exciuding the subsurface areas in and around the structure.

- The subsurface and remaining floor areas 12 and 13 , of building 14 , were throughly investigated and our results aign fairly well with those from the turnikey subcontractor. A tentative hazard assessment on the soil underneath both the walls and remaining floor areas is being prepared. This assessment also includes the footer surfaces that were in the process of being remediated until the structural integrity came into question. Our verification survey was therefore limited to the areas not covered in the scope of the hazard assessment (i.e.. the open excavated areas and the remaining floor surfaces). With the exception of a strip of soil in area 13 (which was subsequently remediated), all excavated areas are below Deparment of Energy soil guidelines for the state of New York. We also coliected data on the areas to be inciuded in the assessment so that we may be able to assist in the process.

If you have any questions or need additional information please call (423) 576-4108.

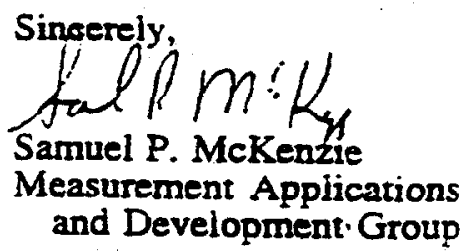

SPM:lec

c: $\quad$ S. K. Armit. BNI

R. D. Foley

File-RC

Qud - Dringing Science to Gife 
owt 10.28 .97

OAK RIDGE NATIONAL LABORATORY

MAMAED BY LOCGTEED MARTIN EREAOY RESEARCH COAPORATION

FOR THE U.S. DEPANTIENT OF ENERCY

POST OFTCE EOX 2000

ON FUDEE TN 3701

October 27, 1997

Dr. W. Alexander Williams

Designation and Certification Manager

Office of Eastern Area Programs

Cloverieaf Building, EM-421

Department of Energy

19901 Germantown Road

Germantown, Maryiand 20874-1290

Dear Dr. Williams:

Contract DE-AC05-960R22464, Independent Verification Survey on Building 14

During the week of September 15, an Independent Verification Survey was conducted on certain areas in Building 14, and a review of Post Remedial Action Data taken from the previously contaminated section on the exterior of Building 31. The results are as follows:

- Building 31 (all interior and exterior surfaces), with the exception of the subsurface, has been determined to be below Deparment of Energy clean-up guidelines.

- In Building 14 we have designated Areas 2,3,4, the hallway areas, all first floor offices (with the exception of 8A and 9A-D), and the Men's and Women's rest rooms as being below Department of Energy criterion.

- The comidor that separates Areas 10 and 11 from Area 12 was surveyed on a previous date. and is below Deparment of Energy clean-up criterion.

- We have reviewed the core sample results taken from Areas 8, 10. and 11 , and conciude that the subsurface of these areas are beiow Deparment of Energy guideiines.

Sincerely,

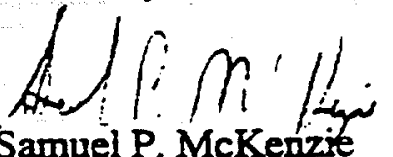

Measurement Applications and Develope Group

SPM:iec

c: S. Armit, BNI

R. D. Foley

File-RC 
OAK AIDGE NATIONAL LABORATORY

MANAGED BY EPOXHEZO MAATN ENEROY RESEARCK COAPORATION

FOA THE U.S. DEPARTMENT OF ENEAGY

-OST OFnCE 80X 2000

onx andere marui

December 22, 1997

Mr. Tim Bymes

CELRB-PP-PM

Army Corps of Engineers

1776 Niagara Street

Buffalo. New York 14207

Dear Mr. Byrnes:

\section{Radlological Verification Surveys on Buidding 14}

During the week of November 10, 1997, an Independent Verifiestion Contractor (IVC) team from Oak Ridge National Libborasory, conducted radiological verification surveys on certain areas in building 14. The rexults are as follows:

1. Area 15 (all interior surfaces and the subsurfates). with the exception of the surface beneach the large experimental tank, adjacent to the pil on the south side of the area. has been decermined in be below reniedial action guidelines.

2. Areas 15A, 15B, and 15B-1 (all interior surfaces and subsurfaces), has been determined to be below remedial action guidelines.

If you have any questions or need further assistance plense call me al (423) $570-7584$

Sincerely.<smiles>CC(C[Te]C1CCCCC1)C1CC1</smiles>

R. D. Foley<smiles>C=CC=CC</smiles>

Measurement Applications<smiles>CC(C)(C)CCCCC(C)(C)C</smiles>
and Development Group

RER:lec

c:

S. Armit. BNl

S. P. MeKenzite

R. E. Rodriguez

Filo-RC 
OAK RIDGE NATIONAL LABORATORY

MANAGED BY LOCKHEED MUATAN ENEAGY AESEAACH CORPORUTON

FOR THE US. DEPAFTIENT OF ENERCY

OST OFFTCE BOX 2008

January 30, 1998

Mr. Tim Byrnes

CELRB-PP-PM

Army Corps of Engineers

1776 Niagara Street

Buffalo, New York 14207

Dear Mr. Bymes:

Independent Verification Survey of Praxair Building 14

During the week of January 12 an Independent Verification Survey was conducted on two areas in Building 14. Also, a review of the "Summary Report for the Process Piping Radiological Investigation Praxair Building 14," has been completed by the independent verification contractor. The results are as follows:

- Area 14 North, with the exception of the cross members associated with the crane rail, all surfaces above six inches from flool/wall interface have been determined to be below the clean-up guidelines as defined in the Department of Energy Order 5400 (wall surfaces to six inches from the floor will be remediated as part of the floor).

- Area 14 Southwest, accessible areas six inches from floor/wall interface up to approximately tweive feet along the south and west walls have also been determined to be below Department of Energy guidelines.

- Area 20A West was determined to be below Deparment of Energy Order 5400.5 guidelines via a review of data collected by the turnikey subcontractor.

We concur with the resuits of the summary of the process piping investigation in Builaing 14 and release this area as below Department of Energy ciean up criterion for this site.

If you need additional information please contact me at (423) 576-7584.

Sincerely,<smiles>C1CC2C3CC1CC2C3</smiles><smiles>[Te]O[Te]</smiles>

R. D. Foley

Measurement Applications and Development Group

RDF:lec
c: $\quad$ S. P. McKenzie
M. E. Murray
R. E. Rodriguez 
$\sin 3-18.4 s$

D-10

OAK RIDGE NATIONAL LABORATORY

MANAGED BY LOCKHEED MARTIN ENERGY RESEARCH CORPORATION

DOR THE U.S. DEPAATMENT OF ENERGY

POST OFFICE BOX 2008

ONK RIDGE TENNESSEE 37031

March 16, 1998

Mr. R. J. Gibbs

Bechtel National. Incorporated

151 Lafayette Drive

Post Office Box 350

Oak Ridge, Tennessee 37831-0350

Dear Mr. Gibbs:

\section{Independent Verification Survey for Building 14}

During the week of March 2. 1998. an Independent Verification Survey (IVS) was conducted on the subsurface soils and bottom portions of the walls in area $14 \mathrm{~N}$ and $14 \mathrm{SW}$ in Building 14.

The subsurface and remaining floor areas in $14 \mathrm{~N}$ and $14 \mathrm{SW}$ were thoroughly investigated and our resuits align well with those of the subcontractor. Tentative supplemental standards for portions of the soil underneath both the walls and knee wall areas, are being developed. With the exception of the locations to be covered by suppiemental standards, areas $14 \mathrm{~N}$ and $14 S W$ are verified not to have residual contamination above the guidelines, as defined in the Department of Energy (DOE) Order 5400.5. This closes out area $14 \mathrm{~N}$ as the other portions were covered in a previous letter. Area $14 S W$ has been verified to be below DOE guidelines with the exception of the overheads and walls above tweive feet. Cleanup activities on these surfaces will be conducted at a later date. I have attached two tables summarizing the radiological cleanup guidelines for unrestricted use as outlined in DOE Order 5400.5. If you have any questions call (423) 576-7584.

Sincereiy,

O. an $x$. 7 aliz

R. D. Foley

Measurement Applications and Development Group

RDF:lec

Attachment: Table 1; Table 2

c: $\quad$ S. P. McKenzie

M. E. Murray

R. Pilon. ACE

File-RC 
Table 1. Applicable guidelines for protection against radiation adapted from DOE Order $\mathbf{5 4 0 0 . 5}$

(Limits for uncontrolled areas)

\begin{tabular}{lcl}
\hline Mode of exposure & Exposure conditions & Guideline value \\
\hline Indoor gamma & Indoor gamma radiation level & $20 \mu \mathrm{Rh}$ \\
(above background) & 20
\end{tabular}

\section{Surface contamination}

Total residual surface contamination ${ }^{b}$
${ }^{238} \mathrm{U},{ }^{235} \mathrm{U}, \mathrm{U}$-natural (alpha emitters)

Beta-gamma emitters
Maximum
Average
Removable

${ }^{232}$ Th. Th-natural (alpha emitters)

${ }^{90} \mathrm{Sr}$ (bets-gamma emitter) Maximum

Average

Removable

${ }^{226} \mathrm{Ra} .{ }^{230} \mathrm{Th}$, transuranics

Maximum

Average

Removable

\section{Radionuclides in soil}

Radionuclide concentrations in soil (generic)

Derived concentrations
Maximum permissible concentration of the following radionuclides in soil above background leveis, averaged over a 100-m² area

$$
\begin{aligned}
& { }^{225} \mathrm{Ra} \\
& { }^{32} \mathrm{Th} \\
& { }^{30} \mathrm{Th}
\end{aligned}
$$

Total uranium

$$
\begin{aligned}
& 15.000 \mathrm{dpm} / 100 \mathrm{~cm}^{2} \\
& 5,000 \mathrm{dpm} / 100 \mathrm{~cm}^{2} \\
& 1.000 \mathrm{dpm} / 100 \mathrm{~cm}^{2}
\end{aligned}
$$

$$
\begin{gathered}
3,000 \mathrm{dpm} / 100 \mathrm{~cm}^{2} \\
1,000 \mathrm{dpm} / 100 \mathrm{~cm}^{2} \\
200 \mathrm{dpm} / 100 \mathrm{~cm}^{2} \\
300 \mathrm{dpm} / 100 \mathrm{~cm}^{2} \\
100 \mathrm{dpm} / 100 \mathrm{~cm}^{2} \\
20 \mathrm{dpm} / 100 \mathrm{~cm}^{2}
\end{gathered}
$$

$5 \mathrm{pCi} / \mathrm{g}$ averaged over the first $15 \mathrm{~cm}$ of soil below the suriace; $15 \mathrm{pCi} / \mathrm{g}$ when averaged over 15-cm-thick soil layers more than $15 \mathrm{~cm}$ beiow the surface

$60 \mathrm{pCi} / \mathrm{g}^{d}$ 
Table 1 (continued)

\begin{tabular}{lcl}
\hline Mode of exposure & Exposure conditions & Guideline value \\
\hline & Soil hot spot criteria & \\
$\begin{array}{l}\text { Guideline for non- } \\
\text { homogeneous con- }\end{array}$ & $\begin{array}{c}\text { Applicable to locations with } \\
\text { an area } 25 \mathrm{~m}^{2} \text {, with signiñ- }\end{array}$ & $\mathrm{G}_{A}=\mathrm{G}_{i}(100 / \mathrm{A})^{1 / 2}$, \\
where \\
$\begin{array}{l}\text { cantly elevated concentrations } \\
\text { addition to the } 1.00-\mathrm{m}^{2}\end{array}$ & $\begin{array}{l}\mathrm{G}_{A}=\text { guideline for "hot } \\
\text { of radionuclides ("hot spots") } \\
\text { guideline)" }\end{array}$ & $\begin{array}{l}\mathrm{G}_{i}=\text { guideline averaged } \\
\text { over a } 100-\mathrm{m}^{2} \text { area }\end{array}$ \\
\hline
\end{tabular}

The $20 \mu \mathrm{R} / \mathrm{h}$ shall comply with the basic dose limit (100 mrem/year) when an appropriate-use scenario is considered.

${ }^{b} D O E$ surface contamination guidelines are consistent with NRC Guidelines for Decontamination at Facilities and Eacipment Prior 10 Release for Unrestricted Use or Termination of Licenses for By-Product. Source. or Special Nuclear Material, May 1987.

'Beta-gamma emitters (radionuclides with decay modes other than alpha emission or spontaneous fission) except ${ }^{90} \mathrm{Sr},{ }^{228} \mathrm{Ra},{ }^{223} \mathrm{Ra},{ }^{227} \mathrm{Ac},{ }^{133} \mathrm{I},{ }^{129} \mathrm{I},{ }^{126} \mathrm{I},{ }^{123} \mathrm{I}$.

DOE guidelines for uranium are derived on a site-specific basis. A total uranium guideline of $60 \mathrm{pCi} / \mathrm{g}$ will be applied at the former Linde site. This corresponds to a ${ }^{233} \mathrm{U}$ concentration of $-30 \mathrm{pCi} / \mathrm{g}$.

-DOE guidelines specify that every reasonable effort shall be made to identify and to remove any source that has a concentration exceeding 30 times the guideline value, irrespective of area (adapted from Revised Guidelines for Residual Radioactive Material at FUSRAP and Remote SFMP Sites, April 1987).

Sources: Adapted from U.S. Department of Energy, DOE Order 5400.5, April 1990; U.S. Department of Energy, Guidelines for Residual Radioactive Material at Formeriy Utilized Sites Remedial Action Program and Remote Surplus Facilities Management Program Sites, Rev. 2, March 1987: and U.S. Department of Energy, Radiological Control Manual, DOE/EH-0256T Rev. 1, April 1994. 
Table 2. Background radiation levels and concentrations of selected radionuclides in soil near Tonawanda. New York

\begin{tabular}{|c|c|c|}
\hline \multirow{2}{*}{$\begin{array}{l}\text { Type of radiation measurement } \\
\text { or sample }\end{array}$} & \multicolumn{2}{|c|}{$\begin{array}{l}\text { Radiation level or radionuclide } \\
\text { concentration }\end{array}$} \\
\hline & Range & Average \\
\hline $\begin{array}{l}\text { Gamma exposure rate at } \\
\text { ground surface }(\mu R / h)^{\circ}\end{array}$ & $8-11$ & 9 \\
\hline $\begin{array}{l}\text { Concentration of radionuclides } \\
\text { in soil }(\mathrm{pCi} / \mathrm{g})^{a} \\
{ }^{238 \mathrm{U}} \\
{ }^{226 \mathrm{Ra}} \\
{ }^{322} \mathrm{Th}\end{array}$ & $\begin{array}{l}0.8-1.1 \\
0.7-1.1 \\
0.5-0.9 \\
\end{array}$ & $\begin{array}{l}1.0 \\
0.9 \\
0.8 \\
\end{array}$ \\
\hline
\end{tabular}

-Values obtained from four locations in the Tonawanda area.

Source: R. E. Rodriguez M. E. Murray, and M. S. Uziel, Results of the Radiological Survey at the Town of Tonawanda Landfill, Tonawanda. New York (TNYOOI), ORNL/RASA-92/12, Martin Marietta Energy Systems, Inc., Oak Ridge Natl. Lab., October 1992. 


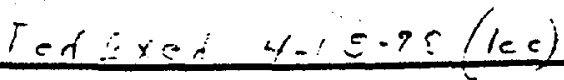

D-14

OAK RIDGE NATIONAL LABORATORY

MANAGED BY LOCKHEED MARTIN ENERGY RESEARCH CORPORATION

FOR THE U.S. DEPARTMENT OF ENERGY

POST OFFICE BOX 2008

OAK RIDGE TENNESSEE 37831

April 15, 1998

Mr. Ray Pilon

CELRB-PP-PM

U. S. Army Corps of Engineers

1776 Niagara Street

Buffalo, New York 14207

Dear Mr. Pilon:

Contract DE-AC05-96OR22464, Independent Verification Interim Letter Reports for Building 14, Linde Site

At your request, copies of all interim letter reports issued at the conclusion of several Independent Verification Contractors surveys are enclosed. The enclosed email (November $19,1997)$ was sent to cover work performed the week of November 10,1997 , on Building 14 at the Linde site. A formal letter report to cover this work will be sent at a later date, of which the email will serve as a basis to the formal letter.

If additional information is needed contact me at (423) 576-7584.

Sincerely,

AR $a_{1}, 0$.

R. D. Foley

Measurement Applications

and Development Group:

RDF:lec

Enciosures 7 (Bldg. 14 Letters)

c: $\quad$ S. P. McKenzie

R. E. Rodriguez

R. E. Swaja

File-RC 
OAK RIDGE NATIONAL LABORATORY

MANAGED BY LOCKHEED MARTIN ENERGY RESEARCH CORPORATION

FOR THE U.S. DEPAFTMENT OF ENERGY

POST OFFICE BOX 2008

OAK RIDGE. TENNESSEE 3783?

May 20, 1998

Mr. Robert J. Gibbs

Bechtel National, Incorporated

151 Lafayette Drive

Post Office Box 350

Oak Ridge, Tennessee 37831-0350

Dear Mr.Gibbs:

\section{Contract DE-AC05-96OR22464, Building 14 Independent Verification Survey}

An independent verification (IV) survey was conducted on walls and overhead structures in area 14SW, Building 14, during the week of May 4,1998. This area of investigation was approximately twelve feet above the floor (all areas below this level were released earlier). This report does not cover the ceiling of the area. An additional area was surveyed, which was a stairway leading to the underground utility tunnels, adjacent to area 12 .

The walls, piping, and other structures were thoroughly investigated and our results align well with those of the subcontractor. Tentative supplemental standards for portions of the lower horizontal I-beam surface, that is closest to the south wall, are being developed. With the exception of the ceiling and locations to be covered by supplemental standards, area $145 W$ is verified not to have residual contamination above the guidelines as defined in Department of Energy (DOE) Order 5400.5. The entrance stairway leading down to the utility tunnel, adjacent to area 12 was also surveyed and contamination above guidelines was detected. This elevated area was reported and additional decontamination was conducted. After additional decontamination the area was verified to be below the DOE order. The enclosed table summarizes the radiological clean-up guidelines for unrestricted use as outlined in DOE Order 5400.5.

If you have questions or need further information please call (423) $576-7584$.

Sincerely,

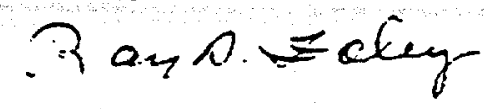

R. D. Foley

Measurement Applications and Development Group

RDF:lec

Enclosures 1

c: S. P. McKenzie

M. E. Murray

R. E. Rodriguez

File-RC 
OAK RIDGE NATIONAL LABORATORY

WNAGED BY LOCKHEED MARTIN ENERGY RESEARCH CORPORATION

A THE U.S. DEPARTMENT OF ENERGY

POST OFFCE BOX 2008

ONK RIDGE. TENNESSEE 37831

June 10. 1998

Mr. Tim Byrnes

CELRB-PP-PM

U. S. Army Corps of Engineers

1776 Niagara Street

Buffalo, New York 14207

Dear Mr. Bymes:

Contract DE-AC05-96OR22464, Independent Verification Survey on Building 14

During the week of June 1, 1998, an Independent Verification (IV) survey was conducted on the walls and certain overhead structures in areas 14SE, Building 14. This area of investigation began approximately one foot above the floor. Additionally, the overheads in area 9 were surveyed, as well as the ceiling in area $14 \mathrm{SW}$.

The walls, piping, and part of the overhead structures in area 14SE were surveyed. This area is verified not to have residual contamination above guidelines defined in the Department of Energy (DOE) Order 5400.5. The exception is an area along the wall that leads to area 15 . This area will be renovated and post remedial surveys will be conducted. The ceiling in area $14 \mathrm{SW}$ and the overheads and ceilings in area 9 were surveyed and both areas were verified to be below DOE guidelines. Enclosed is a table summarizing the radiological cleanup guidelines for unrestricted use as defined in DOE Order 5400.5.

If you have any questions or need additional information call (423) 576-7584.

Sincerely,

R. D. Foley

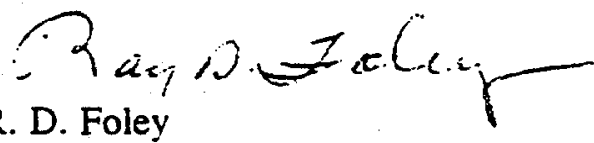

Measurement Applications and Development Group

RDF

Enclosures 1

clenc: $\quad$ R. J. Gibbs. BNI

S. P. McKenzie

R. Pilon, USACE

File-RC 
OAK RIDGE NATIONAL LABORATORY

MANAGED BY LOCKHEED MARTIN ENERGY RESEARCH CORPORATION

FOR THE U.S. DEPARTMENT OF ENERGY

POST OFFICE BOX 2008

ONK RIDGE TENNESSEE 37831

July 7,1998

Mr. Robert J. Gibbs

Bechtel National, Incorporated

151 Lafayette Drive

Post Office Box 350

Oak Ridge, Tennessee 37831-0350

Dear Mr. Gibbs:

Contract DE-AC05-960R22464, Clarification Status of the Independent Verification of Building 14 Praxair Site

This correspondence is intended to clarify/update the status of the Independent Verification of Building 14 at the Praxair site. There are several areas that have been declared below Department of Energy (DOE) Guidelines as defined in DOE Order 5400.5 . Included are the first and second floor offices, areas $2,3,4,8,10,11,13,20 \mathrm{~A}, 20 \mathrm{~A}$ east, 20B, 20B-1, 20C, 21, the corridor, and stairways.

Areas 12 and 14 all have residual contamination in the soils beneath the walls that run along the west wall. Area 15 contains an area beneath a large experimental tank that was inaccessible and could not be surveyed. In addition, difficult to access metal surfaces in the overhead areas of area 14 (lower horizontal surfaces close to wall in area 14SW) are above DOE clean-up criterion. Survey activities are continuing in area 9 and 14 SE, and subsequent letters will address these areas.

Sincerely,

Mpri kigh

Measurement Applications and Deveiopment Group

SPM:lec

Enclosures

c: $\quad$ R. D. Foley

M. E. Murray

File-RC 
OAK RIDGE NATIONAL LABORATORY

MANAGED BY LOCKHEED MARTIN ENERGY RESEARCH CORPORATION

FOR THE U.S. DEPARTMENT OF ENEAGY

POST OFFICE BOX 2008

OAKRIDGE, TENNESSEE 3783 1

September 16, 1998

Mr. R. J. Gibbs

Bechtel National, Incorporated

151 Lafayette Drive

Post Office Box 350

Oak Ridge, Tennessee 37831-0350

Dear Mr. Gibbs:

Contract DE-AC05-960R22464. Independent Verification Surveys of 9 Lab and 14SE of Building 14

During the week of July 6-13. 1998. Independent Verification Surveys (IV) were conducted in areas 9 Lab and 14SE of Building 14. The ceiling, exposed subsurface soils, and remaining floor areas of area 14SE were investigated. This area of investigation includes approximately one foot of the bottom of the wall. In addition the subsurface soils, walls, and remaining concrete floor surfaces were surveyed in area $9 \mathrm{Lab}$.

The exposed soils in areas $9 \mathrm{Lab}$ and 14SE were scanned and soil samples were collected. The remaining concrete floor surfaces were also surveyed. After further decontamination efforts, review of the post remedial action radiological data supplied by the subcontractor, and additional data collected from the remediated areas collected by the independent verification contractor. these areas are verified not to have residual contamination above the guidelines as defined in Department of Energy (DOE) Order 5400.5. Walls in area 9 Lab were also scanned and after removal of additional contaminated material this area has also been verified not to have contamination above DOE guidelines. There is a portion of the subsurface in area 9 Lab containing a pipe that will be included in the request for supplemental standards, as it is above clean-up criterion. This email is not a verification statement and should not be used as one, since our soil samples have not been analyzed.

If you have any questions or need further information please call (423) 576-4108.

Sincerely,

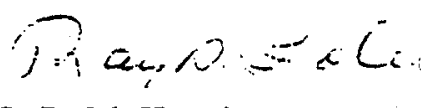

S. P. McKenzie<smiles>C1CCCCC1</smiles>

Measurement Applications and Development Group

SPM:lec

C: R. D. Foley

File-RC 


\section{OAK RIDGE NATIONAL LABORATORY}

\section{OAK RIDGE NATH NAM ENERGY RESEARCH CORPORATION}

FOR THE U.S. DEPARTMENT OF ENERGY

POST OFFICE BOX 2008

OAK RIDGE TENNESSEE 37831

September 21, 1998

Mr. R. J. Gibbs

Bechtel National. Incorporated

151 Lafayette Drive

Post Office Box 350

Oak Ridge, Tennessee 37831-0350

Dear Mr. Gibbs:

\section{Contract DE-AC05-960R22464, Clarification Status of the Independent Verification of Building 14, Praxair Site}

This correspondence is to clarify our standing regarding areas remediated but not covered under previously issued correspondence. Portions of these areas were either omitted from earlier reports or additional decontamination was required. Area 21, which at one time housed underground sumps. has been decontaminated and falls below Department of Energy (DOE) guidelines for soil contamination as outlined in DOE Order 5400.5. The area was at one time enclosed and fell within the scope of this effort. The walls. steps. ceiling, and pipes associated with the stairwell in area 12. that leads to the utility tunnel, was also surveyed and is below DOE guideiines for surface contamination. The internal piping was cleared during the process piping investigations. However, the subsurface is not covered under this letter. Survey data collected by the subcontractor at an area along the wall between areas 14SE and area 15 , have been released as falling below the DOE surface guidelines.

If you have any questions or need additional information call (423) 576-4108.

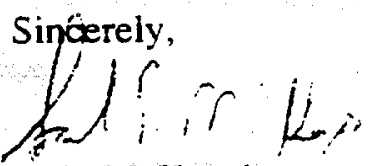

S. P. McKenzie

Measurement Applications

and Development Group

SPM:lec

c: R. D. Foley

File-RC 
OAK RIDGE NATIONAL LABORATORY

MANAGED BY LOCKHEED MARTIN ENERGY RESEARCH CORPORATION

FOR THE U.S. DEPARTMENT OF ENERGY

POST OFFICE BOX 2008

OAK RIDGE. TENNESSEE 3783

October 23, 1998

Mr. Ray Pilon

CELRB-PP-PM

U. S. Army Corps of Engineers

1776 Niagara Street

Buffalo, New York 14207

Dear Mr. Pilon:

Contract DE-AC05-96OR22464, Clarification of Verification Activities Associated With Building 14, Praxair Facility

The purpose of this correspondence is to clarify any outstanding areas and misunderstood issues with respect to verification activities conducted in Building 14 at the Praxair facility. Initially, this type of correspondence served only as a vehicle to expedite the restoration/betterment activities that followed the remediation of parts of this building. They have never been intended as a final verification statement. A final, comprehensive verification report will be issued to cover all Oak Ridge National Laboratory (ORNL) activities in Building 14.

The ceiling in Area 14SE has been determined to fall below the surface guidelines as defined in Department of Energy (DOE) Order 5400.0. All soil samples have now been analyzed and the outstanding subsurface soils in Areas 9 and 14 fall below the site specific soil criterion of $60 \mathrm{pCi} / \mathrm{g}$ total uranium. All surfaces (walls, stairs, floor, ceiling, and electrical conduits) associated with the stairwell leading to the utility tunnel in Area 12 have been verified as falling below the DOE surface guidelines. We are presently awaiting post remedial action surveys of the drain lines and sump location under the floor in this area.

If you have any questions or need more information please call (423) 576-4108.

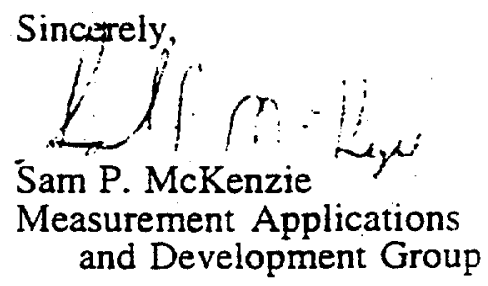

SPM:lec

c: $\quad$ R. D. Foley

R. J. Gibbs, BNI

File-RC 
OAK RIDGE NATIONAL LABORATORY

MANAGED BY LOCKHEED MARTIN ENERGY RESEARCH CORPORATION

FOR THE U.S. DEPARTMENT OF ENERGY

POST OFFICE $80 \times 2008$

ONK AIDGE TENNESSEE 37831

November 4,1998

Mr. Ray Pilon

CELRB-PP-PM

U. S. Army Corps of Engineers

1776 Niagara Street

Buffalo, New York 14207

Dear Mr. Pilon:

\section{Contract DE-AC05-960R22464, Radon Contamination Building 14, Praxair Facility}

The purpose of this correspondence is to convey the results of our investigations for potential radon contamination in building 14, at the Praxair Facility. We are reporting our findings in picocuries per liter ( $\mathrm{pC} / \lambda)$ and not working levels, because our detectors measure radon and not the daughters of radon. The numbers can be converted to working levels, taking into consideration a few basic assumptions.

If you have any questions or need additional information please call (423) 576-4108.

Sincerely,

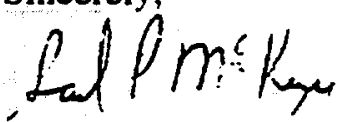

S. P. McKenzie

Measurement Applications

and Development Group

SPM:lec

Attachment: 1 Drawing, Radon Sampling Location, Former Linde Site, Building 14

c: $\quad$ R. D. Foley

R. J. Gibbs. BNI

M. E. Murray

File-RC 


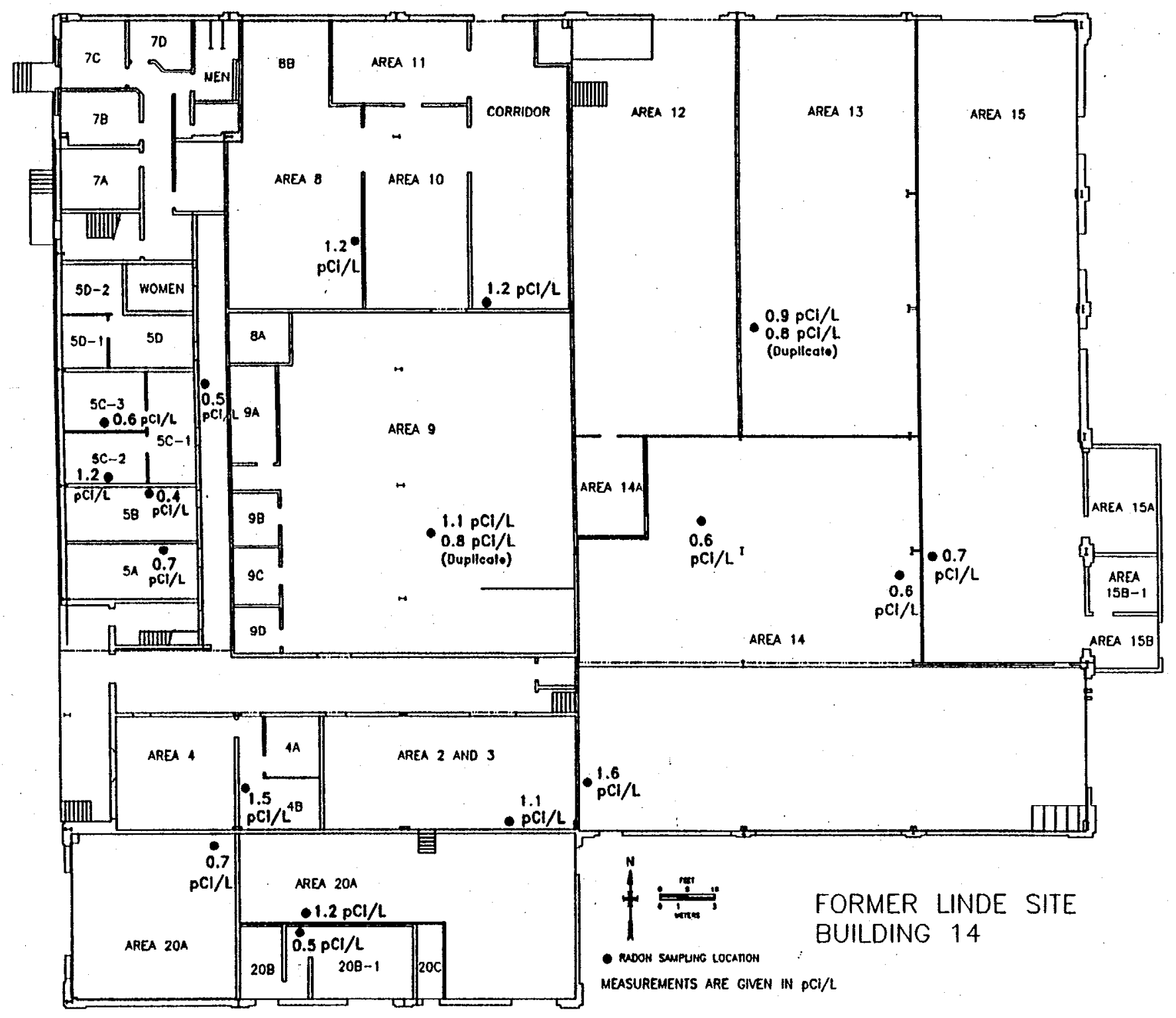

莡

1


OAK RIDGE NATIONAL LABORATORY

MANACED GY LOCKHED MAFTM ENERGY RESEARCH CORPORATION

FOA THE U.S. DEPARTMENT OF ENERGY

POST OFFCE BOX 2008

ONK RIDGE TENNESSEE 37831

November 10, 1998

Mr. Ray Pilon

CELRB-PP-PM

U. S. Army Corps of Engineers

1776 Niagara Street

Buffalo, New York 14207

Dear Mr. Pilon:

Contract No. DE-AC05-960R22464, Radiological Data for Building 14 Praxair Site

The purpose of this correspondence is to convey the results of our evaluation of radiological data, taken by the subcontractor for Building 14, at the Praxair site. We agree with their findings (attached), that the surfaces of the sump located in the utility tunnel section of area 12 , as well as the east and west drain lines leading into the sump, fall below the Department of Energy (DOE) criterion for radiological surface cleanup. The north drain line however, does exceed guidelines and further action will be necessary to resolve this portion of pipe. The remedial action survey of this sump area had not been received until recently. This sump area also falls below the DOE criterion for surface cleanup, and will be addressed in our verification report to be released subsequently.

If you have any questions or need additional information please call (423) 576-4108.

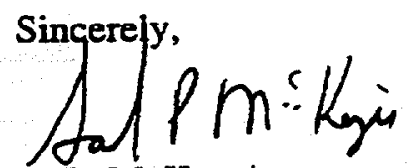

S. P. McKenzie

Measurement Applications

and Development Group

SPM:lec

Attachment

c: R. D. Foley

R. J. Gibbs, BNI

M. E. Murray

File-RC 


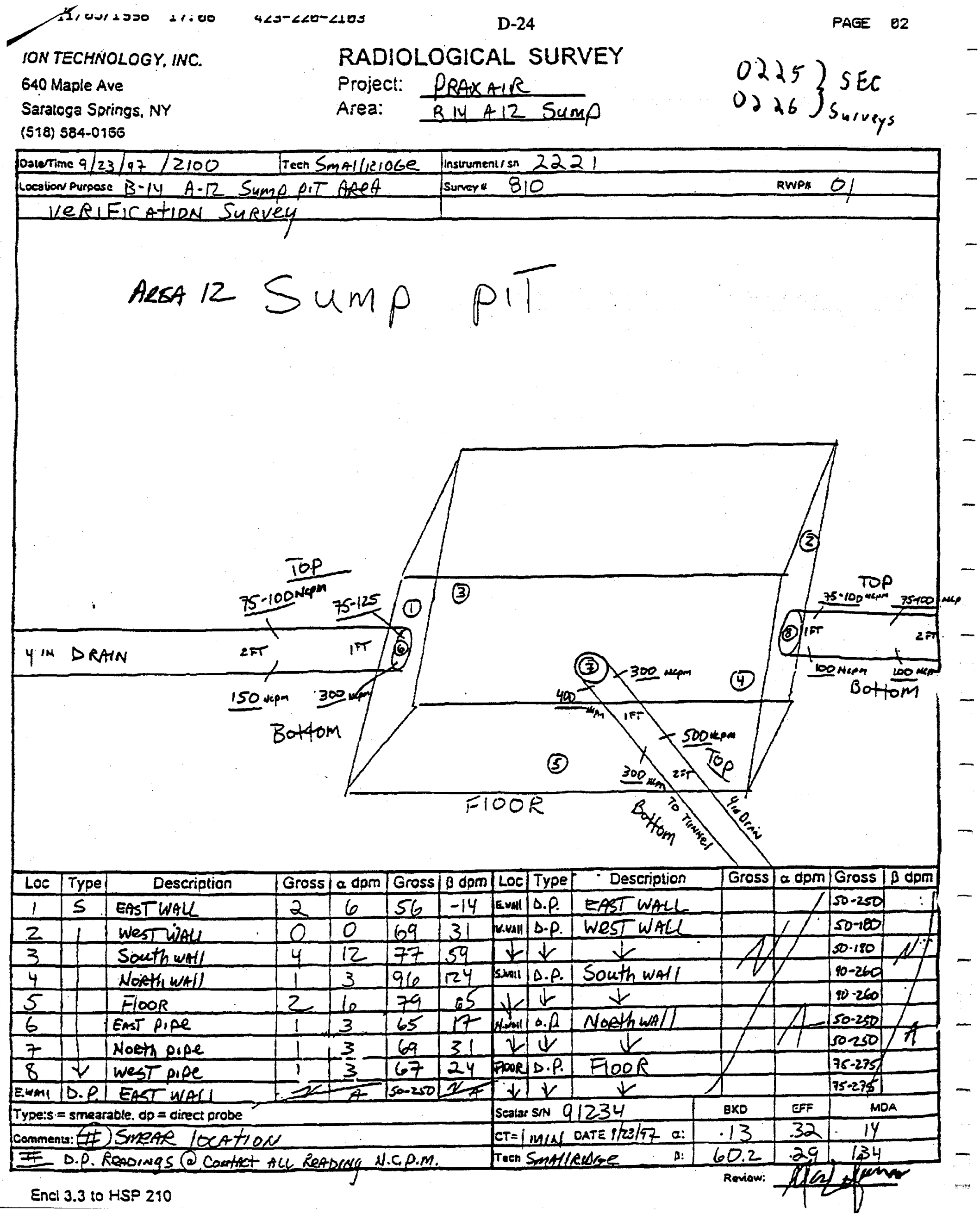




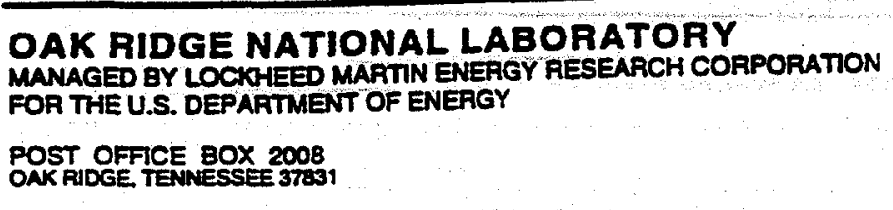

January 13, 1999

Mr. Ray Pilon

CELRB-PP-PM

U.S. Army Corps of Engineers

1776 Niagara Street

Buffalo, New York 14207

Dear Mr. Pilon:

\section{Contract No. DE-AC05-96OR22464, Post Remedial Action Report Review for Building 14}

As you requested, members of the Measurement Applications and Deveiopment group, have reviewed the draft "Post Remedial Action Report for Building 14, Linde Site." This report was prepared by Bechtel National, Incorporated, dated November 1998.

In general we agree with the findings of this report. The data presented in this report agrees with our data within the bounds of radiological field survey techniques and instrument variation. However, there are a few comments we would like to make.

- The term "supplemental limits" is used several times in the document, but nowhere in the document are the "supplemental limits" stated that were used for areas not decontaminated and subsequently hazard assessed. We feel that these values should be included in the document. If different limits were used in different locations, these should also be stipulated.

- In the last paragraph of the executive summary, first line, it is stated that "the remedial action in Building 14 successfully identified all interior surfaces and sub-surfaces within the building footprint exceeding the remedial action criteria through an extensive delineation phase and review of previously collected delineation data." We take exception to the "all" in this statement. This is not to say the statement is incorrect, but based on many years of experience in this line of work, it has been our experience that small areas of contamination, both surface and sub-surface, can be overiooked. This building has had rooms added on and has undergone extensive remodeling since the Manhattan Project involvement was completed. This kind of action often covers up or makes inaccessible, small areas, which could contain some level of contamination. Due to the extensive radiological survey efforts expended in this building. we feel these areas are few and if they exist, would be small. If future maintenance or demolition activities were to inadvertently disturb these possibly existing areas, we feel that any personnel exposure would be very low and would not exceed exposure guidelines, because of the limited number and size of the areas involved. This statement refers only to those areas where contamination may be undiscovered, not to those areas where supplemental limits have been utilized or where suspected contamination may exist. 
Mr. Ray Pilon

Page 2

January 13, 1999

- The "Post Remedial Action Report," is a large and detailed document. It details both verbally and in drawings those areas still contaminated, where a hazard assessment has been utilized, using supplemental guidelines. We feel Figure 5-1 should be a large size color drawing i.e., an E-size, and the activity data should be included on the drawing and be furnished to the property owner for use by the Building 14 facility manager or maintenance personnel. Since the vast majority of the building is clean, the few areas containing or suspected to contain contamination exceeding guidelines, would be color coded for quick reference. The detailed report would still be available if work was going to be done in locations near known contamination.

- There appears to be a discrepancy in Figure 5-1. There are hazard assessed areas not depicted on the drawing. Example Area 12; south end of room on the east, south, and west walls. There is sub-surface contamination at the base of these three wails, which is not shown.

- There is also a contaminated structural beam in Area 14, which exceeds guidelines and was hazard assessed. The beam lies on the extreme western side of Area 14 and is parailel to the wall.

- Until all hazard assessed areas are approved, it is suggested that health physics coverage be provided for any work in these areas.

- There is a question concerning the legend in certain drawings. Example 4.3-1, "Contamination less than 1 meter on walls with a cross-hatched design and, "Contamination up to 1 meter on walls," with a double cross-hatch design. Basically, both say the same thing, so we suspect something has been left out. This legend and any other legends, should be reviewed and clarified.

- The "Post Remedial Action Report," states that some of the hazard assessed areas have been approved and others are pending. It is recommended the document be held until all areas are approved and the document rewritten to reflect these changes.

If you need additional information or have any questions please call me at (423) 576-7584.<smiles>C1CCCCC1</smiles>

Ray D. Foley

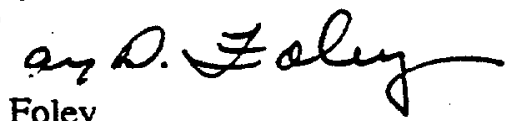

Measurement Applications

and Development Group

RDF:lec

c: $\quad$ S. P. McKenzie

M. E. Murray

R. E. Rodriguez

File-RC 
ORNL/RASA-99/1

DISTRIBUTION

1. R. D. Foley

2-3. S. P. McKenzie

4. M. E. Murray

5. R. E. Rodriguez
6. M. S. Uriel

7-9. MAD Records Center

10. Central Research Library

11. Laboratory Records - RC

12. M. M. Bukhari, Bechtel National, Inc., FUSRAP Department, Oak Ridge Corporate Center, 151 Lafayette Drive, P.O. Box 350, Oak Ridge, TN 37831-0350

13-22. Raymond. L. Pion, CELRB-PP-PM, U.S. Army Corps of Engineers, 1776 Niagara Street, Buffalo, NY 14207-3111

23-24. Office of Scientific and Technical Information, U.S. Department of Energy, P.O. Box 62, Oak Ridge, TN 37831-0062 
